\title{
Understanding Angular Momentum in Hadrons
}

\author{
Kunal Kathuria \\ Charlottesville, VA
}

A Thesis Presented to the Graduate Faculty of the University of Virginia in Candidacy for the Degree of

Doctor of Philosophy

Department of Physics

UNIVERSITY OF VIRGINIA

August, 2013 


\section{Declaration of Authorship}

I, Kunal Kathuria, declare that this thesis titled, 'THESIS TITLE' and the work presented in it are my own. I confirm that:

- This work was done wholly or mainly while in candidature for a research degree at this University.

- Where any part of this thesis has previously been submitted for a degree or any other qualification at this University or any other institution, this has been clearly stated.

- Where I have consulted the published work of others, this is always clearly attributed.

- Where I have quoted from the work of others, the source is always given. With the exception of such quotations, this thesis is my own work.

- I have acknowledged all main sources of help.

Signed:

Date: 
"Humility means not being anxious to be honored by others."

"Eloquence is truth concisely stated." [AC Bhaktivedanta Swami]

If you can't explain it to a six year old, you don't understand it yourself. [Albert Einstein] At the heart of all knowledge lies simplicity of expression. 


\title{
UNIVERSITY OF VIRGINIA
}

\author{
Abstract \\ Department of Physics \\ Doctor of Philosophy \\ by Kunal Kathuria \\ Charlottesville, VA
}

The spin puzzle has been a relatively long-standing unresolved issue in hadron physics. We review and derive, using a wave-packet formalism, the first step preceding all spin sum rules: the connection of the angular momentum operator to the gravitomagnetic form factors of the energy-momentum tensor (EMT). We present our spin sum rule for spin one hadrons and comment on why the deuteron is an important system to study in an effort to understanding hadron spin and interactions better. We will visit a number of illuminating properties of spin seen from the wave packet approach (e.g. consequences/promotion of azimuthal symmetry in momentum space as the general rule for origin independence of angular momentum, merits of the different decompositions in the literature, a brand new way to express angular momentum, common "accepted" mistakes associated with the derivation etc) and will also say why this approach is the necessary starting point for all treatments. Additionally, we will attempt to clear a number of misconceptions surrounding the operators entering the sum rule (what the different relevant operators are, what the decompositions are, the merits of a partonic decomposition, the EMT in the free vs interacting theory, it being canonical vs Belinfante vs symmetric vs conserved etc) with simple and pertinent observations. 


\section{Acknowledgements}

I would like to express my gratitude to my $\mathrm{PhD}$ advisor Simonetta Liuti for her very supportive attitude and very natural and open comportment during my work with her. I am grateful to her for her humble and gracious approach; for considering ideas just on merit without bias and strongly encouraging the originality she sees in her students.

I am also thankful to Gary Goldstein for his collaboration and help in general.

Thanks goes to all my collaborators in work and fellow students for discussions along the way, especially to J. Osvaldo Hernandez.

I would like to say thank you to the physics department for supporting me as a graduate student and helping me earn a $\mathrm{PhD}$ in physics.

I am extremely grateful for the support and presence of Amit Acara dasa and Mayapriya devi dasi, without whom my efforts would have been meaningless, and the rest of my family here, including my wife Prachi.

I owe a big thanks to my parents for indulging my creative desires all along and I also thank my sister, Kamna.

I thank my wife Prachi, the artist, for drawing the figures I needed for this document.

Finally, thank you to Sunil Patel for providing the template for this manuscript. 


\section{Contents}

Declaration of Authorship i

Abstract

Acknowledgements $\quad$ iv

List of Figures viii

List of Tables $\quad$ ix

Abbreviations $\quad x$

Physical Constants $\quad$ xi

1 Introduction 1

1.1 Preface: Writing Style . . . . . . . . . . . . . . . . . . 1

1.2 Introduction . . . . . . . . . . . . . . . . . . . 2

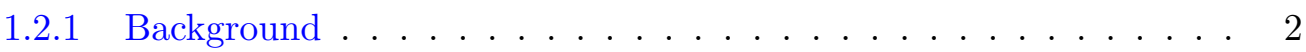

1.2 .2 The Spin Crisis . . . . . . . . . . . . . . . . . . 4

1.2 .3 Current Work . . . . . . . . . . . . . . . . . . . . 6

1.2.4 Specifics on Kinematical Regime . . . . . . . . . . . . . . 7

1.2.5 Light-Cone Coordinates . . . . . . . . . . . . . . . . . . 8

1.2.6 Brief Introduction to Generalized Parton Distributions . . . . . . . 8

1.2.7 Connection with the Energy-Momentum Tensor . . . . . . . . . . . 13

2 Hadronic Spin Decomposition $\quad 14$

2.1 The Two Decompositions and Operators Related to Angular Momentum . 14

2.2 Overview and Wave-Packet Results . . . . . . . . . . . . . . . . . . . 18

2.2 .1 Overview ......................... 18

2.2.2 The Nucleon Spin Sum Rule Developed by X. Ji . . . . . . . . . 19

2.2.3 Effective Zero Orbital Angular Momentum from Symmetry Considerations . . . . . . . . . . . . . 20

2.2.4 Azimuthal Momentum Symmetry is the Criterion for Zero OAM . 21

2.2.5 Necessity of Wave Packets and Comment on Separability of Angular Momentum . . . . . . . . . . . . . . . . . . 23 
2.2.6 Dark Angular Momentum . . . . . . . . . . . . . . . . . . . 24

2.2 .7 Another Observable . . . . . . . . . . . . . . . . . . 25

2.3 A careful wave-packet treatment . . . . . . . . . . . . . . 26

2.3.1 Relating Angular Momentum to the EMT . . . . . . . . . . . . 26

2.3 .2 Derivation of Sum Rule . . . . . . . . . . . . . . . 29

2.3 .3 Obtaining a sum-rule with wave-packets . . . . . . . . . . . . 34

2.3.4 Translation-invariance for momentum and spin and translationdependence for OAM . . . . . . . . . . . . . 35

2.4 Gravitational Form Factors . . . . . . . . . . . . . . . . . . . 37

2.4.1 The Connection with GPDs . . . . . . . . . . . . . 37

2.4.2 Checking Individual Terms for Conservation . . . . . . . . . . . . . 39

3 Deuteron $\quad 42$

3.1 Background . . . . . . . . . . . . . . . . . . 42

3.2 Energy-Momentum Tensor Decomposition . . . . . . . . . . . . . . . . 43

3.2 .1 Trace Anomaly . . . . . . . . . . . . . . . . . . . . 44

3.3 Spin One Sum Rule . . . . . . . . . . . . . . . . . . . . . . . 44

3.4 Helicity Amplitudes: Formalism . . . . . . . . . . . . . . . . . . . 51

3.4.1 Simple Examples of Transformations . . . . . . . . . . . . . . . . . 55

3.4 .2 Accessing the Compton Form Factors . . . . . . . . . . . . . 61

4 Simple Physical Arguments Related to Angular Momentum 62

4.1 Boost-like Operators . . . . . . . . . . . . . . . . . . . . 62

4.1.1 Commutation of Boost and Rotations . . . . . . . . . . . 62

4.1 .2 Degeneracy in Spin . . . . . . . . . . . . . . . . . . 64

4.1.3 Pauli-Lubanski Vector: Contribution to Light-Cone Boost or Angular Momentum? . . . . . . . . . . . . . . . . 69

4.1.4 Good and Bad Components of the Pauli-Lubanski Vector . . . . . 72

4.1.5 Longitudinal and Transverse Angular Momentum for the Nucleon and the Deuteron . . . . . . . . . . . . . . 73

4.1.6 What About Orbital Angular Momentum? . . . . . . . . . . . . 77

4.1.7 What Does a Boost Do Classically and Quantum Mechanically to Angular Momentum? . . . . . . . . . . . . . . . 77

4.1.8 Meaning of Spin and Orbital Contributions in a Stationary State . 82

4.1.9 Why Transverse Angular Momentum Dominates . . . . . . . . . . 84

4.1.10 Angular Momentum Contribution of the Magnetic Form Factor for the Proton and Deuteron . . . . . . . . . . . . . 85

4.1 .11 What Does Boosting Do to Spin Individually? . . . . . . . . . . 86

4.1 .12 Ji's Recent Argument for Gluon Spin . . . . . . . . . . . . . . . . . 86

4.1 .13 Spin-Orbit Coupling . . . . . . . . . . . . . . . . 87

4.1.14 Is There Origin Dependence in Angular Momentum? (On the Density Interpretation) $\ldots \ldots . . \ldots 88$

4.1.15 Covariance of the Off-forward Matrix Element . . . . . . . . . . 88

4.1 .16 Aside: Operator-shifting in Field Theory . . . . . . . . . . . . 89

4.1.17 Comparing the Ji and JM Decompositions of the EMT . . . . . . . 90

4.1.18 Further phenomenological development: Quark-Gluon Separation . 93

4.1 .19 Eigenstates of the Pauli-Lubanski Spin Vector . . . . . . . . . . 93

4.1 .20 Normalization of $T^{\mu \nu} \ldots \ldots \ldots$. . . . . . . . . 94 
4.1 .21 Burkardt's Torque Analysis . . . . . . . . . . . . . . . . . . . . . . . . 95

4.1 .22 On a Comment from Leader's Paper . . . . . . . . . . . . . . . 95

$\begin{array}{llr}5 & \text { Vorticity } & \mathbf{9 7}\end{array}$

5.1 Introduction and Observations . . . . . . . . . . . . . . . . 97

5.1 .1 The Nature of Vorticity . . . . . . . . . . . . . . . . . 97

5.1.2 Extension to Field Theory: Vorticity Density . . . . . . . . . . . . 100

5.1 .3 The Circulation . . . . . . . . . . . . . . . . . 100

5.1 .4 Vorticity is a Conserved Charge . . . . . . . . . . . . . . 102

5.2 Calculations: Relating Vorticity and Angular Momentum . . . . . . . . 103

5.2 .1 Involving Off-diagonal Elements of EMT . . . . . . . . . . . . 103

5.2.2 Writing Momentum as a Function of Vorticity . . . . . . . . . . . 103

5.2.3 Evaluating the Becattini Term: Redefinition of J in terms of vorticity 104

6 Conclusions and Philosophy 107

6.1 Summary of Contributions . . . . . . . . . . . . . . . . . . 107

6.2 Brief Points of Philosophy . . . . . . . . . . . . . . . . . . . . . . . . . . . 110

6.2.1 Research Perspective . . . . . . . . . . . . . . . . . . . 113

$\begin{array}{ll}\text { A Review } & 115\end{array}$

B Derivation of the Vorticity-Angular Momentum Connection 119

C Constructing the Transformation Matrices 122 


\section{List of Figures}

1.1 Relative contributions of quark-spin, quark OAM, gluon spin, gluon OAM (JM) and quark-spin, quark $O A M^{\prime}$, gluon AM (Ji) of the two decompositions of angular momentum of the proton in a simplified model, calculated by M. Burkardt. Each model sums to the same whole: total angular momentum of the proton. . . . . . . . . . . . . . 5

1.2 Deeply Virtual Compton Scattering . . . . . . . . . . . . . . . . 9

2.1 Zero angular momentum of 2-particle system at any time in simple classical example . . . . . . . . . . . . . . . . . . . . . . . . . . . 21

2.2 Gaussian wave-packet centered at $p_{0}^{z} \ldots \ldots \ldots \ldots$. . . . . . . . 31

3.1 (color online) Upper panel: Total angular momentum density distributions, $J_{q}, q=u, d$, calculated using the GPD parametrization of Ref.[6]. Theoretical error bands are included. Lower panel: Orbital angular momentum density distributions, $L_{q}, q=u, d$, obtained from Eq.(3.19), using the parametrizations from $[6]\left(J_{q}\right)$ and [39] $(\Delta q)$. In both panels the dashed lines correspond to the scale $\mu^{2} \approx 0.1 \mathrm{GeV}^{2}$ where spectator models are evaluated [3]; the full lines from our fit results are calculated at $Q^{2}=4 \mathrm{GeV}^{2} \ldots \ldots \ldots \ldots \ldots \ldots \ldots$

3.2 (color online) (Upper panel) Contributions $H+E, H$, and $E$, to the integrand in the angular momentum sum rule, Eq.(3.2). All curves were calculated at the scale $Q^{2}=4 \mathrm{GeV}^{2}$, using the parametrization from Ref.[6] for the free nucleon. (Lower panel) The ratio of nuclear to nucleon contributions to angular momentum, $H_{D} / H_{N}$ (dashes), and $H_{2} /\left(H_{N}+E_{N}\right) \equiv$ $J_{D}(x) / J_{N}(x)$, (full curve), calculated using Eq.(3.16) for the deuteron. The small hatched area represents the experimental results from Ref.[40].

4.1 Parallel/Longitudinal Boost on current loop carrying angular momentum

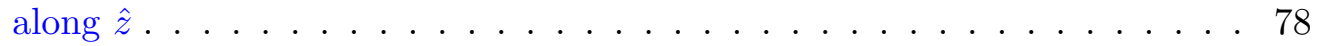

4.2 Transverse (along $-z$ ) boost on current loop carrying angular momentum

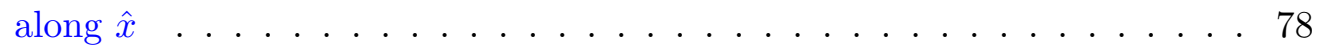

4.3 Boosting homogeneous scalar field of fixed momentum . . . . . . . . . 82

5.1 Cylinder rotates in fluid with vorticity . . . . . . . . . . . . 98

5.2 "Average" vorticity is zero: no rotation of cylinder. Top View. . . . . . . 98

5.3 Uniform field does not have vorticity . . . . . . . . . . . . . . . . . . 99 


\section{List of Tables}

3.1 Unpolarized Helicity Amplitudes (Soft Part) . . . . . . . . . . . . . . . . 60

3.2 Transverse Unpolarized Helicity Amplitudes (Soft Part) . . . . . . . . . . 60 


\section{Abbreviations}

$\begin{array}{ll}\text { AM } & \text { Angular Momentum } \\ \text { CFF } & \text { Compton Form Factor } \\ \text { DIS } & \text { Deep Inelastic Scattering } \\ \text { DVCS } & \text { Deeply Virtual Compton Scattering } \\ \text { EMT } & \text { Energy Momentum Tensor } \\ \text { GPD } & \text { Generalized Parton Distribution } \\ \text { IMF } & \text { Infinite Momentum Frame } \\ \text { OAM } & \text { Orbital Angular Momentum } \\ \text { OPE } & \text { Operator Product Expansion } \\ \text { PDF } & \text { Parton Distribution Function } \\ \text { PL } & \text { Pauli Lubanski } \\ \text { QFT } & \text { Quantum Field Theory } \\ \text { QM } & \text { Quantum Mechanics } \\ \text { WP } & \text { Wave Packet }\end{array}$




\section{Physical Constants}

$\begin{array}{lrl}\text { Charge of electron } & e=-1 \\ \text { Charge of proton } & -e=1 \\ \text { Charge of up-quark } & Q_{u}=+\frac{2}{3} \\ \text { Charge of down-quark } & Q_{d}=-\frac{1}{3}\end{array}$


Without a spiritual dimension to life, life is meaningless. That is to say that if one succumbs to the worldview that the physical world we see is the all-in-all, without subtle core truths, that takes away all the charm there is to be found in anything, including physics. It renders our creative thrills meaningless and very anticlimactically trivialized. Meditation not only stills our mind for better creative space, it makes all other pursuits more spirited, pleasurable, united and cleansed of insecurities and biases. To this end, I have found meditation on a sound vibration to be the most effective. Research shows that certain sounds (like "Ma" to indicate "mother") resonate with us deeply. Similarly, meditative sound vibrations or mantras resonate with our real constitutional make-up and are a formidable meditation technique. Regular mantra meditation helps see through our role-playing, conditioning and external labels. The whole purpose of meditation is to bring the mind under control by giving it a constructive spiritual focus and to get in touch with a deeper dimension of existence - our real selves, beyond these external identifications. I have found the (chanting and focused hearing of) the Hare Krishna mahamantra to be especially effective in my meditation, and it has so greatly enhanced all other pursuits by giving them a common essence that now I can't turn away from it. It goes,

Hare Krishna Hare Krishna Krishna Krishna Hare Hare Hare Rama Hare Rama Rama Rama Hare Hare 


\section{Chapter 1}

\section{Introduction}

\subsection{Preface: Writing Style}

Before we begin, let us make a few notes about the writing style one can expect to encounter in this work. Throughout, I will make an effort to emphasize essential physical points. I will construe rigor as using robust and intuitive physical reasoning, and not unnecessarily involved treatments. The occasions where necessary, the latter will be duly employed. We will consider pertinent examples that illustrate the concept at hand, be they of a classical nature relevant to hadronic physics (like springs or current loops), or drawn from mundane circumstances. For example, one may use the case of a government servant that may think himself/herself free over short intervals of duty, but is really bound to answer to the government. The context will be clear whenever such examples are pertinently employed. Personally, I did not wish to be constrained by a patterned approach, though at no place will physics writing norms be given up. There is a small section on philosophy surrounding this particular work, as, after all "philosophy" means "love of wisdom" and it is wisdom when beholding physical phenomena that we really seek. The use of first person will be limited to this paragraph. Certain diagrams will be hand-drawn for the appropriate pedagogical mood, and for ease of presentation of specific aspects. Finally, from time to time I have inserted some physically interesting "asides," relevant to the physics or formalism being used.

If errors persist in this document, in part due to its lengthy nature, I apologise and seek to revise them. 


\subsection{Introduction}

\subsubsection{Background}

In trying to understand one detail of physical nature, i.e. the structure of the proton, physicists have spent at least 50 years and yet have not arrived at a very good understanding of how the proton's constituents give it its measured quantum numbers. And, consequently, the problem is open for all species of hadrons. The hope is that while attempting to understand the proton, a whole lot of mysteries surrounding quark-gluon interactions/QCD will come to light. Let us, therefore, build up to the project under consideration with very brief rudimentary background from what is known about proton structure.

It has been experimentally shown that the proton is not a single particle with fixed charge. Early experiments showed that when one probes it from a close distance, it behaves like multiple centers of charge assembled together. In the naive quark model, these are simply the three fractionally charged quarks: $u, u \& d$, somewhat like 3 different species of billiard balls. Parton Distribution Functions (PDFs) tell us what the probabilities of hitting the various species of quarks in momentum space would be if these quarks were interpreted as a loose classical assembly, moving very fast together and incapable of exchanging much transverse momentum, at large momentum transfer, $Q^{2}$. However, Generalized Parton Distributions (GPDs), developed by X. Ji and A. Radyushkin [1], give us the framework to interpret the electron-proton Deeply Virtual Compton Scattering (DVCS) cross-section as a sum over different quark and gluon scattering amplitudes, also at sufficiently high virtual photon $Q^{2}$ in the asymptotic limit. Due to the fact that the invariant four-momentum of the virtual photon $(Q)$ is large, the scattering can be phenomenologically viewed as a short-time process, where the constituents are disentangled or loosely bound- though correlated by previously obtaining soft behavior- and are summed over in the cross-section. This is much like a classical spring that obeys Hooke's law: when one pulls in the middle over short distances, parts of the spring seem to be free; however, when one really begins to pull from the end (analogous to long-time viewing), one sees that the part is stiff and very much bound to the rest of the spring.

The first question that can be asked is: how effective is the original quark model? To first order, it describes the hadron we see. Before we consider the model, the only difference between the nature of electron-proton interactions and electron-positron interactions would come from the mass of the proton, if we probe from a distance. It is 
only when we come closer (i.e. at high energies or $Q^{2}$ ) with our probe do we begin to see the complex structure being represented by a single-particle model. When we consider the valence-quark model, we can say that when the probe comes closer, it "sees" the individual quarks closely and is able to affect them individually, thereby breaking apart the proton or releasing new particles from the interaction. From a distance, it merely sees the entangled bound-state represented by an identical-particle wave function. If we introduce on top of this model an "ad-hoc" strong interaction term, the quark wave function would not yield to being altered easily from a distance but would seem to interact freely with the electron only over short times or if the electron were to get very "close". That is to say, when apparently devoid of entanglement with the rest of the nucleus, like two particles in diverse labs are not entangled with each other, the parton's interaction force with the nucleus would be overridden by the probe, which would independently interact with the parton under the parton model. However, as we shall see in the context of angular momentum, this situation is far from how we understand the meaning of "weakly interacting " in quantum mechanics. This is a testament to the subtle, finer nature of hadronic/strong interactions.

Now, let us turn attention to our project - hadronic angular momentum. First we start with the basic picture. How effective are simple proton models in describing its angular momentum? Well, one can start to think of the three valence quarks in the proton to be in the $l=0$ state of orbital motion and in the $S^{z}=\frac{1}{2}$ overall spin state of three spin-half particles. Again, this model has many well-known shortcomings when scattering data are considered. And for another thing, this entanglement picture lies at cross-purposes with the parton model- the first order partonic interpretation, obtaining in the description of PDFs, where the flavored quarks are distinct semi-classical particles arranged in a weak soup in the IMF that are not entangled with each other quantum mechanically. So now we start to introduce cross-talk between effective models. We will really have to admit that to understand OAM and spin decompositions, we will need to consider entanglement between the different contributions, just like a person and their personality are inseparably separable.

Whatever our treatment is, it will be true that the structure of the proton (hadron) can be probed not just via scattering experiments, but also via observables like orbital angular momentum and spin. It should not be surprising therefore, in retrospect, that X. Ji [2] was able to connect the above two quantities at the level of generalized parton distributions (GPDs). In other words, the structure decides both the scattering behavior and the obtaining orbital motion of the partons. This is what structure means, and the 
two things are thus directly correlated.

One must consider the gauge field that is necessary for parton binding, i.e. gluons, what their role in carrying angular momentum is, if spin and orbital contributions lend to separation for quarks and gluons, whether gauge invariance is retained in parts of the operator and whether certain gauges seem to be preferred in the description of this problem in QCD. Phenomenological interpretation of proton data shows that it is overly simplistic to suppose that quarks lie in the inert kinematical state of zero orbital momentum, and this is what we would have guessed given the complexity of proton kinematical structure and partonic content. This is precisely the spin crisis: the spin of the proton is more than twice the individual quark and gluon spin contributions summed together, $\Delta \Sigma$ and $\Delta G[3]$.

\subsubsection{The Spin Crisis}

There are traditionally two ways one decomposes a hadron's spin into its constituents: the Jaffe-Manohar (JM) and the Ji decomposition. Symbolically, they are as follows:

JM: Total AM $=$ Quark-Spin $(\Delta \Sigma)+$ Quark OAM $\left(L_{q}\right)+$ Gluon Spin $(\Delta G)+$ Gluon OAM $\left(L_{g}\right)$

Ji: Total AM $=$ Quark-Spin + Quark $O A M^{\prime}\left(L^{\prime q}\right)+$ Gluon AM $\left(J_{g}\right)$

These terms shall be concretely explained in the next chapter. In the two decompositions, the quark spin term is the only common term and is by itself gauge-invariant. In the JM decomposition, there are no other gauge-invariant terms. Gluon spin is given by the famous $\Delta G$ function, which is physically interpretable only in the $A^{+}=0$ gauge as $E \times A$, the gluon spin, but there are still more subtleties regarding its meaning involving the gauge-link, which we shall not get into here. Suffice it to say that it is not gauge-invariant and does not have a clear meaning like the quark spin term $(\Delta \Sigma)$ does. In the Ji decomposition, each of the 3 terms is gauge-invariant, but the gluon total AM term is not broken into constituents involving OAM and spin. In either case, one seeks a physically meaningful interpretation of the various terms that may shed light on proton structure. Only the quark-spin term has an easy partonic interpretation, i.e. it is directly related to the spin structure function $g_{1}$. The importance of gauge-invariance is generally retained in discussions surrounding the various contributions, with the insistence that physical observables be manifestly gauge-invariant. However, this is not a 

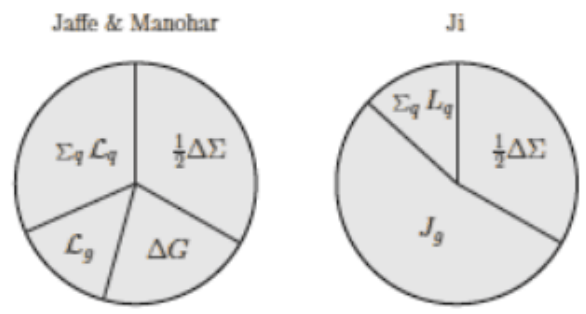

FIgURE 1.1: Relative contributions of quark-spin, quark OAM, gluon spin, gluon OAM $(\mathrm{JM})$ and quark-spin, quark $O A M^{\prime}$, gluon AM (Ji) of the two decompositions of angular momentum of the proton in a simplified model, calculated by M. Burkardt. Each model sums to the same whole: total angular momentum of the proton.

universally accepted notion when dealing with the partonic interpretation of spin, as intimated above. Certain gauges like the light-cone gauge $\left(A^{+}=0\right)$ are often used to give a physical meaning to light-cone operators [4]. Our thinking here is that even though the partonic interpretation stops at leading twist, in some sense one would like the operators to retain their meaning (e.g. quark OAM) and justify why this is so. For this to be the case, gauge-invariance would be a desirable characteristic for the particular contribution in question. In fact, we put forth in our thesis the interpretation that these operators, if correctly formulated, do in a sense represent the physical quantity they advertise and at the same time can only be regarded as inherently coupled to the other constituents. For example, separating quark and gluon contributions may be like separating water and wetness- they are in one sense separable and in another not. Similarly, spin and OAM mix non-trivially under transverse boosts (spin-orbit coupling, really).

Mathias Burkardt calculated the contributions of the pieces of the two decompositions in a simple "QED with gauge-field" model calculation [3] and presented his results in the form of two pie charts:

As part of the present work, we study angular momentum in the deuteron. We would like to study the deuteron for the following reasons:

1) The proton has already been studied in much detail and this is a way to supplement that effort.

2) The deuteron is an isoscalar bound state of a proton and a neutron, the next simplest hadron to consider. One can see how the constituents of angular momentum, partonic 
content etc. change for two nucleons taken together as opposed to one. This is done by expressing deuteron structure functions in terms of the nucleon structure functions.

3) We would like to compare the spin-one Generalized Parton Distributions (GPDs) that describe the spin of the deuteron to those of the nucleon, and see how they are each connected to $G_{M}$, the respective magnetic form factor(s) of the hadron.

4) We would like to compare and contrast the twist and structure functions in the two cases for both longitudinal and transverse spin.

The deuteron is a spin-one bound system consiting of one proton and one neutron, i.e. six valence quarks. The Pauli exclusion principle would effectively not allow a spin singlet state since this would require a spatially symmetric wave function for the proton and neutron; however, when the two are close, their finer structure and interactions determine their wave function and therefore it is not a priori required that there be such a state. In fact, no spin singlet bound state of this kind exists.

\subsubsection{Current Work}

Following are the focal areas of this work undertaken to gain a better understanding of hadron spin. The main results and conclusions will be briefly summarized in the last chapter.

1) A holistic wave-packet treatment to understand the origin of the spin sum rule, and to employ it to (re)derive these sum rules for the nucleon and the deuteron. We will look at all the interesting physical properties that arise from considering such states in special cases of symmetry, boosts, rest frame, momentum eigenstates etc.

2) Developing a spin sum rule for the deuteron; studying the spin and OAM partonic content in the deuteron and comparing the results to the nucleon; studying the different structure functions that do and do not enter the deuteron sum rule and commenting on their physical meaning; understanding more deeply how scattering/partonic helicity amplitudes and angular momentum are connected.

3) Understanding how simple physical transformations like boost affect spin and OAM, and total AM, in QCD; connecting these to their classical and non-relativistic quantum 
mechanical analogs to gain a deeper understanding of spin.

4) Efforts to calculate photon-target helicity amplitudes for the deuteron after developing a clean formalism for them that is in analogy with the proton case, with an aim to separate the momentum and helicity dependent terms from each other; locating an observable that may give easier access to the partonic content figuring in the spin 1 sum rule.

5) Studying a new observable that may deepen our understanding of angular momentum properties of hadrons-the field vorticus motion or field vorticity, and relating it to the energy-momentum tensor via symmetry and conservation properties of the tensor; commenting on the physical meaning of this preliminary study.

\subsubsection{Specifics on Kinematical Regime}

Let us first establish the conditions under which the parton model obtains in Compton Scattering. At the end we will add the intuitive ad hoc assumption of Feynman that the hadron's constituents must be moving with very high momentum in the so-called infinite momentum frame (IMF). Calling $p^{\mu}=\left(E, \overrightarrow{0}_{\perp}, p^{3}\right)$ the momentum of the incoming electron and $p^{\prime \mu}=\left(E^{\prime}, \vec{p}_{\perp}, p^{\prime 3}\right)$ the momentum of the outgoing electron, we calculate $Q^{2}$. Clearly, the outgoing electron is free to pick up transverse momentum.

$$
\begin{aligned}
q^{2} & =2\left(E^{\prime}-E\right)^{2}-\left(p^{\prime 3}-p^{3}\right)^{2}-p_{T}^{2} \\
& =2\left(p^{3} p^{\prime 3}-\sqrt{\left(p_{3}^{\prime 2}+p_{T}^{2}\right)\left(p_{3}{ }^{2}\right)}\right. \\
& =2\left[p^{3} p^{\prime 3}-p^{3} p^{\prime 3}\left(1+\frac{p_{T}^{2}}{2 p_{3}^{\prime 2}}+\ldots\right)\right] \\
\therefore Q^{2} \equiv-q^{2} & =p_{T}^{2} \frac{p_{3}}{p_{3}^{\prime}}+m^{2} \frac{p_{3}^{\prime}}{p_{3}}+m^{2} \frac{p^{3}}{p_{3}^{\prime}}
\end{aligned}
$$

This shows that for $Q^{2}$ to be large, the incoming and outgoing electron momenta not only have to be large, but one has to be significantly larger than the other. If they are about the same order, since transverse momentum is not usually big, $Q^{2}$ will not tend 
to be very large.

\subsubsection{Light-Cone Coordinates}

For an extensive review of this topic, one is referred to [4]. Light-cone coordinates appear naturally when dealing with QCD in the infinite momentum frame (as does the lightcone gauge $A^{+}=0$ ). The matrix elements of the light-cone operators and of light-cone components of Lorentz 4-vectors carry natural physical facility and interpretation in the IMF. To first order, it is fair to say that the same matrix elements one would evaluate in Cartesian coordinates in the IMF are easier and natural to calculate in their lightcone version. It is said, though, that "QCD lies on the light cone," which gets us into subtler points we shall not get into in this section. One example of this is the question whether the $0^{\text {th }}$ or the + component should be used to define angular momentum of the quarks and gluons in the hadron correctly. X.Ji uses the former canonically safe version, especially for an operator which transforms non-covariantly since it is a cross-product (AM), whereas Jaffe-Manohar prefer the latter [5].

Summarily, we will define light-cone coordinates and the general Lorentz inner product here. Given a vector $v^{\mu}=\left(v^{0}, v^{1}, v^{2} v^{3}\right)$,

$$
\begin{array}{r}
v^{+} \equiv \frac{1}{\sqrt{2}}\left(v^{0}+v^{3}\right) \\
v^{-} \equiv \frac{1}{\sqrt{2}}\left(v^{0}-v^{3}\right) \\
\vec{v}^{\perp} \equiv\left(v^{1}, v^{2}\right) \\
v^{\mu} w_{\mu}=\left(v^{+} w^{-}+w^{+} v^{-}\right)-\vec{v}^{\perp} \cdot \vec{w}^{\perp}
\end{array}
$$

\subsubsection{Brief Introduction to Generalized Parton Distributions}

Generalized Parton Distributions (GPDs) give us insight into the structure and partonic content of the proton (actually any hadron). This will be a physically motivated introduction since the details of GPD structure are not presently relevant. For an extensive treatment, the reader is advised to refer to Diehl's thorough review [4], from which the properties given below are referenced. For what are known as chiral-even GPDs, one 


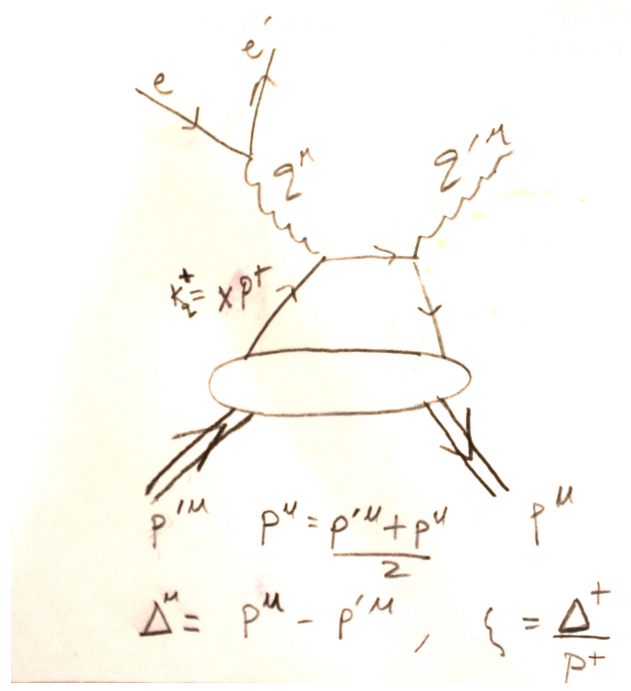

Figure 1.2: Deeply Virtual Compton Scattering

starts with the scattering amplitude of Deeply Virtual Compton Scattering (DVCS) referred to commonly as the Compton Amplitude. The process is:

$e P \rightarrow e^{\prime} P^{\prime} \gamma$

and is illustrated in the diagram below. The kinematical labels are defined via the figure with the addition $Q^{2} \equiv-q^{2}$, the invariant 4-momentum of the photon squared. The kinematical regime suitable to GPDs is the same as that for ordinary parton distribution functions (PDFs), i.e. the Bjorken limit: $Q^{2} \rightarrow \infty, P . Q \rightarrow \infty$ where $\frac{Q^{2}}{P . Q}$ remains finite. The latter can be rewritten in the rest frame of the proton as $M . \nu$, where $M$ is the mass of the proton and $\nu$ is the "energy" of the virtual photon in the rest frame of the proton.

The Compton Amplitude is first canonically obtained from first principles of field theory, and then treated in the regime of the "handbag diagram" shown below. The fully operator product expanded version of the latter in terms of functions of successive twists is performed, and these structure functions of different twists are what are known as GPDs. For example, the Compton Amplitude rewritten up to twist-2 OPE GPDs reads $[6]$ :

$$
T^{\mu \nu}=\frac{1}{2} g_{T}^{\mu \nu} \mathcal{F}_{S}^{\Lambda \Lambda^{\prime}}+\frac{i}{2} \epsilon_{T}^{\mu \nu} \mathcal{F}_{A}^{\Lambda \Lambda^{\prime}}
$$


where $g_{T}^{\mu \nu}=g^{\mu \nu}-p^{\mu} n^{\nu}-p^{\nu} n^{\mu}, \epsilon_{T}^{\mu \nu}=\epsilon_{\alpha \beta \sigma \rho} g_{T}^{\alpha \mu} g_{T}^{\beta \nu} n^{\rho} p^{\sigma} \equiv \epsilon^{-+\mu \nu}$, and $p$ and $n$ are the unit light cone vectors. The labels $S$ and $A$ refer to the coefficients of the symmetric and antisymmetric components of the Compton Amplitude in its two Lorentz indices. They also exhibit the same (anti)symmetry in the "non-helicity-flip" positive and negative helicity amplitudes [6]. This "amplitude" is contracted with photon polarization vectors, $\epsilon^{\mu}, \epsilon^{\prime * \nu}$ to obtain a physical number that can be interpreted in DVCS experiments, $f_{\Lambda_{\gamma}, \Lambda ; \Lambda_{\gamma}^{\prime}, \Lambda^{\prime}}$, where the indices $\Lambda$ represent the helicities of the photon and proton in the initial and final state, respectively. This is similar to how real and scalar helicity amplitudes are formed in DIS by contracting these objects with the Hadronic Tensor, $W^{\mu \nu}$.

The twist-2 chiral-even GPDs are defined via the following bilocal vector and axial currents:

$$
\begin{aligned}
& F_{\Lambda, \Lambda^{\prime}}^{S}(x, \zeta, t) \\
= & \int \frac{d \kappa}{2 \pi} e^{i x P^{+} \kappa}<p^{\prime}, \lambda^{\prime}|\bar{\psi}(-k n) \gamma \cdot n \psi(k n)| p^{\prime}, \lambda^{\prime}> \\
= & \frac{1}{2 \bar{P}^{+}}\left[\bar{U}\left(P^{\prime}, \Lambda^{\prime}\right)\left(\gamma^{+} H(x, \zeta, t)+\frac{i \sigma^{+\mu}\left(-\Delta_{\mu}\right)}{2 M} E(x, \zeta, t)\right) U(P, \Lambda)\right] \\
& F_{\Lambda, \Lambda^{\prime}}^{A}(x, \zeta, t) \\
= & \int \frac{d \kappa}{2 \pi} e^{i x P^{+} \kappa}<p^{\prime}, \lambda^{\prime}\left|\bar{\psi}(-k n) \gamma \cdot n \gamma^{5} \psi(k n)\right| p^{\prime}, \lambda^{\prime}> \\
= & \frac{1}{2 \bar{P}^{+}}\left[\bar{U}\left(P^{\prime}, \Lambda^{\prime}\right)\left(\gamma^{+} \gamma_{5} \widetilde{H}(x, \zeta, t)+\gamma_{5} \frac{-\Delta^{+}}{2 M} \widetilde{E}(x, \zeta, t)\right) U(P, \Lambda)\right]
\end{aligned}
$$

There are some basic properties of GPDs that are worth mentioning[4]:

1) They are real functions of 3 chosen variables. Here, they are the momentum fraction $X(x)=\frac{k^{+}}{P^{+}}, \zeta=\frac{\Delta^{+}}{P^{+}}, t=\Delta^{2}$ (GGL parametrization, further details found in [6]). Also, $H(x,-\zeta, t)=H(x, \zeta, t)$. 
2) Their first moments give the basic proton form factors for each quark (Dirac, Pauli, axial, pseudoscalar). For each quark,

$$
\begin{aligned}
& \int H^{q}(x, \zeta, t) d x=F_{1}^{q}(t) \\
& \int E^{q}(x, \zeta, t) d x=F_{2}^{q}(t) \\
& \int H^{q}(\tilde{x}, \zeta, t) d x=g_{p}^{q}(t) \\
& \int E^{q}(\tilde{x}, \zeta, t) d x=g_{A}^{q}(t)
\end{aligned}
$$

3) Forward limits are the unpolarized and polarized parton densities:

$$
\begin{gathered}
\int H^{q}(x, 0,0) d x=q(x) \\
\int H^{q}(\tilde{x}, 0,0)=\Delta q(x)
\end{gathered}
$$

4) They are Lorentz-invariant functions, i.e. they do not change with the frame of reference. This is similar to the behavior of Dirac $\gamma$ matrices- they are "universal" objects in the sense of frame and the specific process as well.

5) They evolve like PDFs, via the Altarelli-Parisi equations.

6) They figure in spin sum rules, which shall be discussed at length in the following chapters. This is because they are connected to the matrix elements of the energy momentum tensor, and how this is is shown in the first section of the next chapter.

One can think of the GPDs as functions that parametrize (since they need to be fit to experimental data and are not given by first principles due to the mathematically non-solvable nature of QCD) our ignorance of the soft interactions inside the hadron. Just like the PDFs parametrize the proton structure to first order, GPDs parametrize 
proton structure at a softer/subtler level. PDFs tell us what the partonic content of the proton is, i.e. the "density" of the various quark flavors, if the proton were seen only as an assembly of quarks free of each other's influence, and moving together very fast in a "partonic soup." If this is the relatively true picture, then these are the right functions to think about. GPDs go deeper than PDFs in two ways: they are more formally and robustly obtained; and they work at the amplitude level. The latter means that one can see the GPDs as defining the amplitude that a particular quark exits to virtually interact with the incoming photon, and then after said interaction goes back into the proton. We know the initial and final state, and we are summing over all possibilities at the amplitude level, like a path integral. All paths (helicities) of the quarks and proton are summed over to yield the final amplitude. Physically, this makes sense as a firstorder picture (i.e. picture in the absence of gluons propagating out from the soft part to exchange momentum with the quark) because the electromagnetic interaction between an electron and a hadron can only occur to lowest order with a quark via the electromagnetic vertex, which must interact with the electron and be "absorbed" back subtly by the proton to yield the observed final state. This interaction is virtual because the proton is a bound state of quarks, and it would be unnatural for the quark to become "fully unbound," even at short time scales, to interact with the electron. The short-timescale nature of the interaction is exhibited in the independent virtual quark vertices, each representing the first-order contribution of the interaction of the particular flavor field within the proton with the electron. This would have been a very sensible model in the absence of deeper information anyway, even without the operator product expansion (OPE). However, the OPE gives us this insight into hadron structure provided by GPDs.

The total helicity amplitude of proton-photon scattering in DVCS can be rewritten as a product (convolution) of the quark-proton and quark-electron amplitudes as follows [6]]:

$$
f_{\Lambda_{\gamma}, \Lambda ; \Lambda_{\gamma}^{\prime}, \Lambda^{\prime}}=\sum_{\lambda, \lambda^{\prime}} g_{\lambda, \lambda^{\prime}}^{\Lambda_{\gamma}, \Lambda_{\gamma}^{\prime}}\left(X, \zeta, t ; Q^{2}\right) \otimes A_{\Lambda^{\prime}, \lambda^{\prime} ; \Lambda, \lambda}(X, \zeta, t)
$$

where $g_{\lambda, \lambda^{\prime}}^{\Lambda_{\gamma}, \Lambda_{\gamma}^{\prime}}$ represents the subprocess $\gamma^{*}+q \rightarrow \gamma+q$, i.e. the virtual photon scattering off a quark with polarization $\lambda ; A_{\Lambda^{\prime}, \lambda^{\prime} ; \Lambda, \lambda}$ is the quark-proton helicity amplitude, and the convolution integral is represented by $\otimes \rightarrow \int_{-(\zeta+1)}^{1} d X$. 


\subsubsection{Connection with the Energy-Momentum Tensor}

This review is subsumed in the next chapter, under the third subsection "Nucleon Spin Sum Rules of X. Ji." 


\section{Chapter 2}

\section{Hadronic Spin Decomposition}

\subsection{The Two Decompositions and Operators Related to Angular Momentum}

The canonical energy-momentum tensor (EMT) is the conserved charge associated with infinitesimal translations from the Lagrangian. $T^{0 \nu}$, for each value of the index $\nu$, is a separately conserved charge, the energy and the three spatial momenta of the state respectively. In the free theory (no gluons), it is given by:

$T_{C, \text { free }}^{\mu \nu}=\frac{i}{2}\left(\bar{\psi}(x) \gamma^{\mu} \overrightarrow{\partial_{\nu}} \psi(x)+\bar{\psi}(x) \gamma^{\mu} \overleftarrow{\partial_{\nu}} \psi(x)\right)$

It is not symmetric as it stands. One symmetrizes it thus:

$T_{B, \text { free }}^{\mu \nu} \equiv \frac{1}{2}\left(T_{C, \text { free }}^{\mu \nu}+T_{C, \text { free }}^{\nu \mu}\right)$

One can call this the Belinfante EMT for the free theory. The total angular momentum is given by the appropriate cyclic component of the rank-3 tensor, $J^{k}=\frac{1}{2} \epsilon^{i j k} M^{0 j k}$,

$M^{\mu \nu \lambda}=x^{\nu} T^{\mu \lambda}(x)-x^{\lambda} T^{\mu \nu}(x)+\frac{1}{2} \bar{\psi}(x) \gamma^{\mu} \sigma^{\mu \nu} \psi(x)$,

where the second axial term accounts for the spin possessed by the quarks. This we shall refer to as $M_{C, \text { free }}^{\mu \nu \lambda}$. It is of the form " $\vec{x} \times T^{0} i$ (orbital-term) + spin-term." By effecting the same symmetry as above in the QCD Lagrangian, the canonical EMT for the interacting theory is obtained. So, the Belinfante EMT is generally formed by adding to 
this canonical EMT a piece that ensures the conservation of all four-momentum charges and that also makes it symmetric in its two indices. This piece is typically written as a 4-divergence, and is antisymmetric in the two surviving indices [7].

$\left[T_{B}^{\mu \nu} \equiv T_{C}^{\mu \nu}+\partial_{\beta} X^{\beta \mu \nu}\right.$

This is true of symmetrizing it in the interacting theory as well [7]. JM quote the fully interacting $M^{\mu \nu \lambda}$ in QCD (symmetry of infinitesimal rotations), after they throw away a superpotential term that does not contribute to its expectation value:

$M_{Q C D}^{\mu \nu \lambda}=\frac{i}{2}\left(\bar{\psi} \gamma^{\mu}\left(x^{\nu} \partial^{\lambda}-x^{\lambda} \partial^{\nu}\right) \psi+\frac{1}{2} \bar{\psi}(x) \gamma^{\mu} \sigma^{\lambda \nu} \psi(x)-2 \operatorname{Tr}\left(F^{\mu \alpha}\left(x^{\nu} \partial^{\lambda}-x^{\lambda} \partial^{\nu}\right) A_{\alpha}\right)+\right.$ $2 \operatorname{Tr}\left(F^{\mu \lambda} A^{\nu}+F^{\nu \mu} A^{\lambda}\right)$

They state that the terms correspond to quark orbital angular momentum and spin, and gluon angular momentum and spin, in that order. Only the axial quark-spin term, $\Delta \Sigma$ is gauge-invariant. The difference between canonical/Belinfante versions of $M^{\mu \nu \lambda}$ is not directly relevant to the present discussion (canonical is not directly quoted for the interacting theory, is it? Only $J_{C}$ is generally stated) at hand since they both yield the same expectation value, but they can be seen in[7]. This can be written using the Belinfante version of the EMT in a simple form, as they indeed do write:

$M^{\mu \nu \lambda}=x^{\nu} T_{B}^{\mu \lambda}-(\nu \leftrightarrow \lambda)$.

We will now analyze the difference between the two forms of Ji and JM. If one takes the component $M^{012}$, one arrives at Ji's $J^{z}$, expressed as $J_{q}+J_{g}$, where

$\vec{J}_{q}=\frac{1}{2} \int \psi^{\dagger} \vec{\Sigma} \psi d^{3} x-i \int \psi^{\dagger}(\vec{x} \times \vec{D}) d^{3} x$ and

$J_{g}=\int \vec{x} \times \vec{E} \times \vec{B}$.

Here $E$ and $B$ are obtained canonically from the gauge field and $\vec{D}$ is gauge-covariant derivative. In Ji's version, the two contributions to $J^{q}$ and the gluon contribution $J^{g}$ are each separately gauge-invariant. Most notably, there is a gauge-field interaction term in the orbital motion of the quarks as defined by Ji, which was absent in the JM case. 
This is precisely the term that renders the quark orbital angular momentum, as defined here, separately gauge-invariant.

Note that if $T^{\mu \nu}(x)$ were written in a form that was not symmetrized in $\mu, \nu$ (e.g. $T^{\mu \nu}(x) \equiv T^{\mu \nu}(x)_{q}+T^{\mu \nu}(x)_{g}$, with $\left.T^{\mu \nu}(x)_{q} \equiv \frac{i}{2} \bar{\psi}(x) \gamma^{\mu} \vec{D}^{\nu} \psi(x)\right)$, then by inserting this in the definition of $M^{\mu \nu \lambda}(x)$ in order to obtain the appropriate component $J^{i}$, we would only recover the quark orbital term of Ji, $i \int \psi^{\dagger}(\vec{x} \times \vec{D}) d^{3} x$ (and no quark-spin term). Clearly, this difference would not be accounted for in the gluon contribution, regardless of how it is written. Thus, it makes sense that the Belinfante and symmetric versions of the EMT overlap with each other.

One should notice that Ji's gluon contribution $J_{g}$ is identical as a whole, as Ji alludes, to Eqn (6.35) in JM, where JM take the component $M^{0 j k}$ for pedagogical reasons. Eqn (6.34) from the same paper makes it clear that $J_{g}$ of $\mathrm{Ji}$ is the same as the frequently quoted $S_{g}+L_{g}$ from JM's decomposition, if the "0" component is chosen. They are not inherently different, up to a third piece that disappears in pure gauge theory, since the pieces comes with an interpretation of gluon spin and orbital angular momentum from pure gauge-field theory. The axial quark-spin term for Ji and JM is also identical. Thus, the expectation value of whatever is left, JM's quark-orbital contribution, must be the same as Ji's leftover piece in $J_{q}$, i.e. $J_{q}-\Delta \Sigma$, the latter being the quark-spin term (expressed as an antisymmetrized axial term by JM).

We notice that this seems to imply that the orbital contribution taken from JM's $M_{q}^{012}$ is identical to Ji's! But they clearly differ by an interaction term. What is going on here? JM, when quoting their $M_{Q C D}^{\mu \nu \lambda}$, seem to have absorbed the gauge-field-interaction term in the third subtle $J_{g}$ piece mentioned above [8], allowing the orbital operators to be different. At any rate, the important point is that the interacting-field term does not contribute to the expectation value of the operator at twist two, as gluon GPDs do not enter at this level.

We cannot, however, a priori state that the two orbital contributions are the same at twist 2 for the reason that JM identify the $M^{+12}$ component with $J^{z}$, not $M^{012}$. JM identify the quark contribution with

$\frac{i}{2}\left(\bar{\psi} \gamma^{+}\left(x^{\nu} \partial^{\lambda}-x^{\lambda} \partial^{\nu}\right) \psi+\frac{1}{2} \bar{\psi}(x) \gamma^{+} \sigma^{\nu \lambda} \psi(x)\right)$, using $\gamma^{+}$. 
It is true the above does not make a difference to the spin contribution, but could affect the orbital (note: it is persuasively argued using parity arguments that the orbital component is also unaffected [3], i.e. $\left.M_{q-O A M}^{012}=M_{q-O A M}^{+12}\right)$. Of course, the interactngfield term, $i \bar{\psi} A^{\mu} \psi$, is ignored by Ji when formulating his sum rule, which includes only twist-two quark GPDs and no gluon GPDs, rendering the operator effectively identical to JM's. Moreover, then, this is effectively the angular momentum operator of the free quark theory summed with that of the free gluons from pure gauge theory! Just that it is sandwiched between fully interacting hadron states. This kind of "free" operator picture resembles the weakly interacting phenomenology of quarks and gluons where they are in one sense free of each other's influence when the time-interval in question is small. However, things in QCD are more subtle, as we know and shall show. ${ }^{1}$

It should be noted that JM use the $M^{+12}$-component in the partonic interpretation, even though the total's expectation value is unaffected whether one uses + or 0 , or canonical or Belinfante. However, the form of the operator is affected by both the component chosen and whether the Belinfante or canonical version of the EMT is used in defining $M^{\mu \nu \lambda}$. As stated before, there is no apparent difference between the Ji and JM orbital terms if the interaction term in Ji's quark orbital part is dropped. One should also remind oneself that if angular momentum were a covariant operator, the + component and the 0 component would a priori, for the total and partonically, give the same result in the infinite momentum frame.

$J^{z}=M^{012}$ can be broken into quark and gluon spin/orbital components, as applicable. Ji breaks it as quoted above. In his original paper, he quotes another (primed) decomposition, which frees the quark contribution completely (so it is identical to the sum of JM's quark spin and orbital terms) and absorbs the gauge field in $E$, which has the same explicit form otherwise as $J_{g}$ quoted above [2]. This is related to Burkardt's observation quoted above.

Leader claims [7] that $J_{J M, q}^{z}=J_{J i, q}^{z}$ for the nucleon generally and subsequently that this holds for all components, not just the longitudinal. What does this mean in light of the above? This result must be checked and is contradicted by Burkardt's pie chart [3]. One must also see whether this is true at all twists or just at leading twist.

\footnotetext{
${ }^{1} \mathrm{~A}$ small note is made here that in the definition of $M^{\mu \nu \lambda}(x)$ as $x^{\nu} T_{B}^{\mu \lambda}-(\nu \leftrightarrow \lambda)$, that $x^{\mu}$ must commute (contrary to a popular misplaced notion with regard to Hilbert space commutativity!) with the derivative in $T^{\mu \nu}(x)$. Our derivation yields the right result.
} 


\subsection{Overview and Wave-Packet Results}

\subsubsection{Overview}

Let us now remind ourselves of work that has already been done in hadronic angular momentum treatments before we proceed with the wave packet formalism. There are five stages to the derivation and application of the angular-momentum sum rule, as follows.

1) The expectation value of angular momentum is related to that of the energy-momentum tensor(JM).

2) There is a form-factor decomposition of this latter matrix element into Lorentz structures and the relating of them to actual numbers after sandwiching the appropriate angular momentum component between forward physical states. This gives a sum rule in terms of the EMT structure functions. This has been attempted by both Ji and JM, where the physical states used were helicity states and $J^{z}$ was purportedly calculated.

3) Finally, a connection is made between the matrix elements of the EM-tensor above and those defining GPDs in DVCS (Ji), to cast the same sum rule in terms of GPDs.

4) The angular momentum operator itself is decomposed into quark and gluon spin/orbital components. This has been done differently by JM and Ji. Ji related some of these components to quark GPDs, specifically the quark OAM.

5) Among all this, Harindranath [9] (and later [3]) have attempted to calculate the physical quantities entering angular momentum, like quark spin/OAM via a wave-function treatment under specific models. Harindranath and Kundu use a dressed quark model to represent a proton, whereas Burkardt's model does not involve gluons and does not allow for incorporation of $Q^{2}$ evolution.

We will redo 1) in a holistic wave packet treatment and give reasons as to why this is necessary. We point out a number of physically illuminating points stemming from this approach and related observations. We also stress many pertinent points that are overlooked in this important step, and address many misconceptions surrounding the various steps. We have already provided our decomposition of step 2), specifically for 
the deuteron, in [10]. We have also derived our GPD spin sum rule in 3), again for the deuteron. We clearly reviewed above all the operator forms entering step 4) to clear any confusion surrounding the meaning and usefulness of the various EMT and angular momentum operators and decompositions. We also give our comments regarding the various physical decompositions and present a new way to recast/consider angular momentum (vorticity). In a future work, we will present a pie chart for the deuteron as in 5) (e.g. quark OAM "density" has already been presented in [10]).

The phenomenological points we address below are further developed in later sections.

\subsubsection{The Nucleon Spin Sum Rule Developed by X. Ji}

Let us briefly review the work of X. Ji in developing the proton spin sum rule. Up to twist 2, the EMT for the nucleon can be decomposed into all possible Lorentz structures in the following way:

$$
\begin{aligned}
\left\langle p^{\prime}\left|T^{\mu \nu}(0)\right| p\right\rangle & =\bar{u}\left(p^{\prime}\right)\left[A_{q, g}\left(\Delta^{2}\right)\left(\gamma^{\mu}\left(p+p^{\prime}\right)^{\nu}+\gamma^{\nu}\left(p+p^{\prime}\right)^{\mu}\right)+i B_{q, g}\left(\Delta^{2}\right)\left(\left(p+p^{\prime}\right)^{\mu} \sigma^{\nu \alpha}\right.\right. \\
& \left.+\left(p+p^{\prime}\right)^{\nu} \sigma^{\mu \alpha}\right) \Delta_{\alpha} \frac{1}{2 M}+C_{q, g}\left(\Delta^{2}\right)\left(\Delta^{\mu} \Delta^{\nu}-g^{\mu \nu} \Delta^{2}\right) \frac{1}{M} \\
& \left.+\bar{C}_{q, g}\left(\Delta^{2}\right) g^{\mu \nu} M\right] u(p)
\end{aligned}
$$

Defining $P^{\mu}=p^{\mu}+p^{\prime \mu}$ and using the fact that $T^{++}(0)=\left(P^{+}\right)^{2} \int x d x \int \frac{d k}{2 \pi} e^{i x k P^{+}} \bar{\psi}(-k n) \gamma . n \psi(k n)$ [4], one can connect the gravitomagnetic form factors to the GPDs in the following way by cancelling out the factors of $P^{+}$that appear on either side. After equating coefficients of identical structures in identically valued matrix elements, and then taking the forward limit, we obtain,

$$
\begin{aligned}
& A(0)=\frac{1}{2} \int x d x H(x, 0,0) \\
& B(0)=\frac{1}{2} \int x d x E(x, 0,0)
\end{aligned}
$$

This result holds, of course, for all Lorentz components of $T^{\mu \nu}(0)$, even though the ' ++ ' component was used in the derivation. According to Ji's original paper [2], 
$\left.\left\langle p m=+\frac{1}{2}\left|J_{q, g}^{z}\right| p m=+\frac{1}{2}\right\rangle=A_{q, g}(0)+B_{q, g}(0)=\frac{1}{2} \int d x x H(x, 0,0)+E(x, 0,0)\right)$

And the quark orbital content could then be obtained by substracting from above the longitudinal spin density, $\int d x g_{1}(x)$ or $\int d x \Delta q(x)$ :

$L_{q}^{z}=\frac{1}{2} \int d x x H(x, 0,0)+E(x, 0,0)-\int d x \tilde{H}(x, 0,0)$

These are X.Ji's main early results related to the spin crisis.

\subsubsection{Effective Zero Orbital Angular Momentum from Symmetry Con- siderations}

When we consider a hadron at rest, we see a state that has a definite angular momentum projection even though its total momentum is zero. To this end, let us look at a classical example. Imagine two particles going in planar clockwise orbits of the same radius, side by side [Figure 2], such that at any time they have opposite linear momentum. They are in a continuous beat of adding to give zero momentum, as they revolve at the same frequency. Yet, these constituents each carry identical positive angular momentum. Similarly, quarks and gluons also add to give overall zero linear momentum to the hadronic state in the rest frame, yet they add to give non-zero angular momentum. Now, the real situation of partons contributing to a hadron's angular momentum (AM) is much more intricate than this one but the basic idea is clearly illustrated: for a multi-particle state, zero linear momentum does not imply zero orbital angular momentum (OAM). If a situation of this kind is possible to construct classically, one can only imagine what nature can do in more subtle (with more DOF) situations.

One can counter with a quantum-mechanical entangled two-particle state where the operator $p_{1}+p_{2}$ necessarily evalutates to zero. Eigenstates of total momentum $p$ in muliparticle interacting states can be expressed as a sum (integral) of the eigenstates of the individual particles, so that in total momentum space we have a fixed momentum $p$ :

$\Psi=\sum_{l} e^{i l x_{2}} e^{i(p-l) x_{1}}$.

Here, individually, 


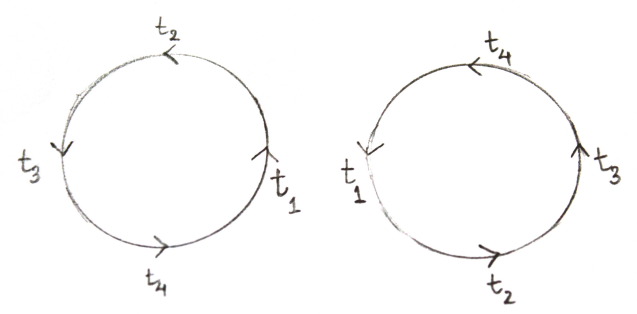

Figure 2.1: Zero angular momentum of 2-particle system at any time in simple classical example

$\left\langle p_{1}\right\rangle,\left\langle p_{2}\right\rangle \neq 0$, but

$\langle\Psi|L| \Psi\rangle=\left\langle\Psi\left|r_{1} \times p_{1}+r_{2} \times p_{2}\right| \Psi\right\rangle=0$,

since $p_{1}$ and $p_{2}$ are opposite. So, multi-particle eigenstates of zero linear momentum yield zero OAM, and furthermore there is no OAM in the direction of linear momentum. However, the general formalism is different in field theory, where the interactions are not abstracted in a potential, are field-based, non-perturbative, and hence we cannot define the orbital angular momentum as $r \times \overrightarrow{T^{o i}}$ unless dealing with the free theory. The QM particle formalism is constrained under non-relativistic first quantization and hence yields results that are not practical, even if one considered the effect of spin-orbit coupling.

\subsubsection{Azimuthal Momentum Symmetry is the Criterion for Zero OAM}

Let us explore OAM a bit further in the field theoretical context. We will see below that the expectation value of AM for a general state, evaluated with a wave packet in momentum space, can be broken into two distinct antisymmetrized terms involving the EMT.

$$
\begin{aligned}
\left\langle J^{\nu \lambda}\right\rangle & =\lim _{\mathbf{k} \rightarrow \mathbf{0}}(-\mathbf{i}) \int \mathbf{d}^{\mathbf{3}} \mathbf{p} \phi(\mathbf{p})\left\langle\mathbf{p}\left|\mathbf{T}^{\mathbf{0 \lambda}}(\mathbf{0})\right| \mathbf{p}\right\rangle \frac{\partial}{\partial \mathbf{k}_{\nu}} \phi^{*}(\mathbf{p}+\mathbf{k})-(\lambda \leftrightarrow \nu) \\
& +(-\mathbf{i}) \int \mathbf{d}^{\mathbf{3}} \mathbf{p}|\phi(\mathbf{p})|^{\mathbf{2}} \frac{\partial}{\partial \mathbf{k}_{\nu}}\left\langle\mathbf{p}+\mathbf{k}\left|\mathbf{T}^{\mathbf{0 \lambda}}(\mathbf{0})\right| \mathbf{p}\right\rangle-(\lambda \leftrightarrow \nu)
\end{aligned}
$$

where $\phi(p)$ represents the momentum distribution of the general state. The second of these disappears when an azimuthally symmetric wave packet is used, as pointed out 
in [11]. This wave-packet state may remind us of azimuthally symmetric hydrogenic wave-functions in the $l=0$ state. Since the second term vanishes with an azimuthally symmetric choice of packet (in total momentum space), we conclude that this term must be purely an OAM contribution. It is for this reason that only the first term enters the commonly quoted sum rule relation, since eigenstates of longitudinal momentum - equivalent to azimuthally symmetric peaked wave packets in total momentum- are considered [5].

This is purely an orbital contribution since spin would not disappear independent of the spin content of the individual momentum eigenstates. And since it disappears for all composite states in all field theories, spatial wave-function symmetry is no longer the appropriate necessary condition for this term to disappear. In fact the condition is promoted from spatial azimuthal symmetry to total momentum azimuthal symmetry for multi-particle states. The generalized rule becomes: When a state has azimuthal symmetry in total-momentum space, its angular momentum is independent of origin. We show this below. Alternatively, when the effective spatial wave-function of the state (like a hadronic wave function, defined as the IFT from total momentum distribution) is azimuthally symmetric, AM is independent of origin.

We show that a state's AM is independent of origin if its momentum-space wave packet is azimuthally symmetric. This of course is inclusive of the rest frame. Shifting the general state by $a^{\nu}$, one obtains

$$
\begin{aligned}
\left\langle J^{\nu \lambda}\right\rangle_{\text {shift }} & =\lim _{k \rightarrow 0} \int d^{3} x d^{3} p d^{3} p^{\prime} \phi(p) \phi\left(p^{\prime}\right)\left\langle p^{\prime}\left|x^{\nu} T^{0 \lambda}(x-a)-(\lambda \leftrightarrow \nu)\right| p\right\rangle \\
& =(-i) \lim _{k \rightarrow 0} \frac{\partial}{\partial k_{\nu}} \int d^{3} p \phi(p) \phi(p+k)\left\langle p+k\left|T^{0 \lambda}(0)\right| p\right\rangle \\
& -a^{\nu} \int d^{3} p|\phi(p)|^{2}\left\langle p\left|T^{0 \lambda}(0)\right| p\right\rangle-(\lambda \leftrightarrow \nu)
\end{aligned}
$$

The first term is independent of the shift, whereas the second term contains it. The second term disappears in the rest frame or if the wave-packet $\phi(p)$ is azimuthally symmetric even up to a phase (e.g. uniformly small or zero transverse momentum), since it contains the expectation value of transverse momentum. This is further elaborated upon in the context of shifting these angular momentum eigenstates in Chapter 4 (this sentence is heavy until one reaches that chapter). This shows that the partons are conspiring to create a state that has properties of purely spin in these cases. The corresponding 
single-particle (or non-composite multiparticle) states (e.g. hydrogen atom in an s-wave state) would be devoid of orbital angular momentum and would be independent of a shift of origin. Spin, being a pure dipole moment, should not change with an origin shift.

From the above, one can conclude that if the wave packet is azimuthally symmetric but peaked about some longitudinal momentum, the partons are stubborn to a change in their AM projection along this longitudinal direction when the origin is shifted. This is unlike their behavior in other kinds of states (those incorporating transverse momentum components in addition, where in the simplest analysis a particle would necessarily gain components in the longitudinal direction via cross-product). We showed that azimuthal symmetry should be considered in momentum space so that the its use in the context of OAM can be extended to composite or multiparticle states. All the results above are unchanged for states boosted along the direction of angular momentum measurement after the boost.

\subsubsection{Necessity of Wave Packets and Comment on Separability of An- gular Momentum}

This is why it is very important to consider the effect of wave packets. They provide the necessary rigor to derive the sum rule that has been used universally. Without it, we would get nonsensical results and infinities for standard expectation values, e.g. Ch. 6 footnote in [5], awkward delta functions in normalization, the standard angular momentum commutation relations failing as these are simultaneously the eigenstates of OAM with zero eigenvalue (shown below) etc. Also, translation invariance would give us an infinite term in the shift and we would not be able to identify the above interesting properties of general Hilbert space states. Off-forward conservation of currents like the quark momentum (considered in OPE of $T^{\mu \nu}(0)$ ) get a physical meaning in the context of wave packets, as such conservation is necessary if the current is to be conserved in a general wave-packet state. The proof showing that the transverse Pauli-Lubanski vector reduces to transverse angular momentum also relies on this treatment, and there are many other instances. It is all right to proceed from wave packets to arrive at whatever expression is appropriate for the limiting case at hand, like plane waves, but this must be our starting point for all treatments. This is also shown in another simple-minded example preceeding the derivation of the sum rule using wave packets below. 


\subsubsection{Dark Angular Momentum}

We give motivation here to the term "spin crisis," termed so as people were puzzled as to the origin of the extra contributions to proton spin beyond partonic spins. This was based on the fact that the parton model incorporates mostly longitudinal-momentumcarrying quarks with spin, and therefore it would be surprising that they would contribute orbital angular momentum in the longitudinal direction (but the difference, one needs to consider, is of the order $h$ ). However, as we know from measuring the spin of the proton, they clearly do!

Since there are clearly contributions to the proton's AM beyond partonic spin, it may be termed dark OAM. In light of the above, it is dark also in the sense that it behaves a lot like spin due to the collective subtle interaction of the partons, for appropriate wave-packet states. Moreover, in a purely free partonic picture, we would not be able to conjecture where it comes from since OAM seems to disappear in this picture altogether for, e.g., a state at overall rest. Therefore the nature of QCD enters the formulation and attempted resolution of this problem.

Let us also remember that the spin of the entire proton is measured without scattering, whereas the partonic spins are measured during shorter times. The proton spin is measured to be far greater than the partonic spins added together totally. At short time scales, we are looking at relatively free, perturbative partons whose OAM contributions are otherwise indicated (in a QM formalism) to be nonexistent and thus their AM would not totally add up to the proton's spin. Things may be different in QCD, and the how is exactly what we are after.

In the same vein, it seems premature to talk of OAM and spin separation from the operator decomposition. The spin that is measured is for short-time interactions. Since it is motivated above that there is no OAM in perturbatively treatable states at zero momentum, and since the spins don't add up to give the hadron's total momentum (as shown in experiment), we are necessarily talking of two different regimes. The field theory formalism does not accommodate this. One is the fully interacting picture and the other is the short-time picture. One may motivate this by thinking of a spring obeying Hooke's law: it is stiff when one pulls it at the end but (short-scale) portions of it are amenable to being freely pinched and pulled. The spin is not measured for the fully interacting hadron. What we know to be quark spin in the non-interacting theory cannot be a priori stated to retain its meaning in the long-time picture. If this is done, 
it must be explained how OAM would not obey this ansatz. Or, indeed, whether such a separation in the theory is permissible. Incidentally, a hint to this is given in JM's AM decomposition. If we call $\bar{\psi} \gamma^{0} \vec{r} \times \partial^{\mu} \psi$ the OAM density, this operator will be zero whenever $\bar{\psi} \gamma^{0} \partial^{\mu} \psi$, the momentum density, is zero, which would only be true in the free theory.

Demanding this separation has its roots in the formalism of quantum mechanics. Here, operators are separable and have a separate meaning for OAM and spin, even if states are entangled. In field theory (classically), one sees that spinors rotate/transform as one object. Even so, in free field theory, the operators for OAM and spin have particular distinguishable forms, for both fermions and gauge fields. However, in the interacting theory, these forms need not retain their original meaning of quark OAM, quark spin, gluon OAM etc. This is manifestly shown by the fact that these forms are not gaugeinvariant [5]. What lies at the crux of the issue is the fact that operators have a fixed meaning independent of the state in question, as long as the state lies in the same theory. The interacting theory and the free theory are not the same theory, and due to the simplification of the states in the free theory, the meaning of the operator reduces to something simple. This is easily seen for the gluon spin and OAM operators.

In fact, these different contributions can be thought to be at once the designated quantity (spin, OAM) and inseparably tied to the total angular momentum of the hadron, much like an electron is at once inseparable from the global electron field and a particular excited instance of it. We shall develop this further later.

Let us also remember throughout that we are looking for purely QCD effects on angular momentum. This is illustrated by the fact that there is no electromagnetic charge in the Lagrangian or operators: we want to know how the strong interaction causes the partons to orbit, that's all. It's a different matter that the probe we are using is electromagnetic, just like for collapsing a wave-function. If the probe has high $Q^{2}$, we are looking at short-time phenomenological manifestations of kinematical quantities, just like for perturbative structure.

\subsubsection{Another Observable}

More recently, other decompositions have been suggested that appear more robust in terms of canonical definition of the operators, but may or may not retain Ji's original gauge invariance. Our purpose here- as far as this issue goes- is not to give a 
new decomposition, but provide some phenomenological partonic insight and revisit the meaning of the decomposition(s). The simplest seemingly complete decomposition [5] is not gauge-invariant term by term, and the one that is [2] is not universally satisfactory in the assignment of appropriate physical meaning to the operators (e.g quark OAM). We claim that the separation of spin and OAM, or quark and gluon contributions, is not theoretically mandated since one is inherently coupled to the other and will give intimations as to a new observable altogether later on. To this end, we perform a general wave packet calculation (like in II C) of the expectation value of the curl of the EMT (or the vorticity of the momentum density), and relate it to AM (refer to Appendix B).

$$
\begin{aligned}
\left\langle\int d^{3} x \frac{-x^{2}}{2} \nabla \times T_{B e l}^{0 k}(x)\right\rangle^{i} & =\frac{1}{2}\left\langle J^{i}\right\rangle+\frac{1}{2}\left\langle J^{i}\right\rangle \\
& =\left\langle J^{i}\right\rangle
\end{aligned}
$$

Thus we have found a new way to express the angular momentum operator, i.e.

$J^{i}=\varepsilon_{\mu \nu \alpha \beta}^{i j k} \partial_{j} T_{B}^{0 k} x^{2}$

[Ji quark-assignment is quark-contribution, and implicitly inclusive of gluonic effects that support quarks, and are not explicit for themselves as gluon AM]

Let us now proceed to topics surrounding our wave-packet derivation.

\subsection{A careful wave-packet treatment}

\subsubsection{Relating Angular Momentum to the EMT}

Let us review how one calculates the expectation value of the spatial components of the energy-momentum tensor, $T^{0 i}(x)=P^{i}(x)$, the momentum-density operator. Here, $\Delta=p-p^{\prime}$. The state $\mid p, s>$ can be defined such that one takes a standard Lorentz boost in the direction of $\mathbf{p}$ from the rest frame spinor that has $\hat{z}$-projection of spin, $s$ (canonical state), or in any other way. Since we rely on the translation properties of 
eigenstates of momentum, the relations that follow are unaffected. So, at least for now, we will drop the spin index in what follows.

Let us introduce at this point the generic wave-packet state, which we will construct as a linear superposition of momentum eigenstates using a wave packet. The state is given by:

$$
|\Phi\rangle=\int d^{3} p \phi(p)|p\rangle
$$

where $\phi$ is any weighting function. For example, it could be centered at $p=p_{o}$, $\phi(p)=\mathcal{N} \frac{1}{a} \exp -\frac{\left(p-p_{o}\right)^{2}}{a}$.

$$
\begin{aligned}
\int d^{3} x d^{3} p d^{3} p^{\prime} \phi(p) \phi^{*}\left(p^{\prime}\right)\left\langle p^{\prime}\left|T^{0 i}(x)\right| p\right\rangle & =\int d^{3} x d^{3} p d^{3} p^{\prime} \phi(p) \phi^{*}\left(p^{\prime}\right) e^{i\left(p-p^{\prime}\right) \cdot x}\left\langle p^{\prime}\left|T^{0 i}(0)\right| p\right\rangle \\
& =\int d^{3} x \delta\left(p-p^{\prime}\right)\left\langle p^{\prime}\left|T^{0 i}(0)\right| p\right\rangle \\
& =\int d^{3} x|\phi(p)|^{2}\left\langle p\left|T^{0 i}(0)\right| p\right\rangle \\
& =2 p_{o}^{0} p_{o}^{i},
\end{aligned}
$$

if the wave packet is chosen to be a standard 3-dimensional delta function in $p$, peaked at $p_{o}: \delta^{(3)}\left(p-p_{o}\right)$, before the last line. We have used Ji's decomposition for the EMT at $x=0$ and obtained the last line. The reason for explicitly translating the operator will become clear in a moment. Also, using the wave packet above we avoid "normalization garbage" like unfitting delta functions, since a normalizing wave packet was used.

In the spirit of Jaffe-Manohar, let us calculate the expectation value of $J^{i}$ in a general state of momentum $p$ of any hadron. Then one can consider the specific cases of longitudinal or transverse momentum, longitudinal or transverse spin projection. Define [5]

$\mathbf{M}^{\mu \nu \lambda}=\mathbf{x}^{\nu} \mathbf{T}^{\mu \lambda}(\mathbf{x})-\mathbf{x}^{\lambda} \mathbf{T}^{\mu \nu}(\mathbf{x})$

One defines, sticking to the Jaffe-Manohar (JM) notation, the expectation value of $J^{i}$ through the Fourier transform of $M^{\mu \nu \lambda}$ at space-time point $x$ : 


$$
\mathcal{M}^{\mu \nu \lambda}=\lim _{k \rightarrow 0} \int d^{3} x e^{i \mathbf{k} \cdot \mathbf{x}}\left\langle\mathbf{p}^{\prime} \mathbf{s}^{\prime}\left|\mathbf{M}^{\mu \nu \lambda}\right| \mathbf{p s}\right\rangle
$$

$J^{3}=M^{012}$ etc. and in general $J^{i}=\frac{1}{2} \epsilon_{i j k} M^{0 j k}$. The expectation value for a stationary state is independent of time and thus can be calculated at any time $x^{0}$. All vectors (including those using Greek indices) used are understood to be three-vectors, so we'll drop the bold-type on them now (four-vectors, if used, will be explicitly made clear). The normalization chosen is $\left\langle p^{\prime}\right| p>=2 p^{0} \delta\left(p^{\prime}-p\right)$. Note in the preceding that initial and final spin will not be the same.

The $k \rightarrow 0$ limit is understood in the following, and taken at the end,

$$
\begin{aligned}
\mathcal{M}^{\mu \nu \lambda}= & \int d^{3} x e^{i k x}\left\langle p^{\prime}\left|x^{\nu} T^{\mu \lambda}(x)-(\lambda \leftrightarrow \nu)\right| p\right\rangle \\
& =\int d^{3} x(-i) \frac{\partial}{\partial k_{\nu}}\left(e^{i k \cdot x}\right)\left\langle p^{\prime}\left|T^{\mu \lambda}(x)\right| p\right\rangle-(\lambda \leftrightarrow \nu) \\
& =\int d^{3} x(-i) \frac{\partial}{\partial k_{\nu}}\left(e^{i k x}\right)\left\langle p^{\prime}\left|e^{i p^{\prime} x} T^{\mu \lambda}(0) e^{-i p x}\right| p\right\rangle-(\lambda \leftrightarrow \nu) \\
& =\int d^{3} x(-i) \frac{\partial}{\partial k_{\nu}}\left(e^{i k x} e^{i\left(p^{\prime}-p\right) x}\right)\left\langle p^{\prime}\left|T^{\mu \lambda}(0)\right| p\right\rangle-(\lambda \leftrightarrow \nu) \\
& =\int d^{3} x(-i) \frac{\partial}{\partial k_{\nu}}\left(e^{i\left(k+p^{\prime}-p\right) x}\right)\left\langle p^{\prime}\left|T^{\mu \lambda}(0)\right| p\right\rangle-(\lambda \leftrightarrow \nu) \\
& =\lim _{k \rightarrow 0} \lim _{p^{\prime} \rightarrow p}-i\left[\frac{\partial}{\partial k_{\lambda}} \delta^{3}\left(k+p-p^{\prime}\right)\right]\left\langle p^{\prime}\left|T^{\mu \lambda}(0)\right| p\right\rangle \\
& +\delta^{3}\left(k-p+p^{\prime}\right) \frac{\partial}{\partial k_{\nu}}\left\langle p^{\prime}\left|T^{\mu \lambda}(0)\right| p\right\rangle-(\lambda \leftrightarrow \nu) \\
= & \lim _{k \rightarrow 0} \lim _{p^{\prime} \rightarrow p}-i\left[\frac{\partial}{\partial k_{\nu}} \delta^{3}(k)\right] \mid{ }_{k=0}\left\langle p\left|T^{\mu \lambda}(0)\right| p\right\rangle
\end{aligned}
$$

How one changes the momentum $p^{\prime}$ of the final ket in taking the derivative of the matrix element affects the physical meaning of the ket and the evaluation of the matrix element (not the sum rule, as we shall derive and see). One may simply change it in the canonical state (a state generally obtained by boosting the rest-frame spinor, originally in an eigenstate of spin along $z$, by the desired momentum), or some other preferred general state. Basically, one must choose a generalized form for the ket that holds for all momenta and in this we have already made the choice of how we are going to change state with respect to transverse momentum. The prescription in the sum rule states that one just change the variable $p^{\prime}$ in the transverse direction in taking the derivative and leave the rest of the components be. This generalized mathematical form will decide the final result. This is because there are an infinite number of ways to have the same 
eigenvalue of momentum but a different state of spin (and thus kets representing this). If one took the limit $\lim _{p^{\prime} \rightarrow p}$ one would end up with the original physical state, of course.

Note that in the last step above we defined $k$ to be independent of $\Delta$. If one does not do this one cannot go from line(2) to line(3) since one is left with an extra term. Incidentally $\mathrm{JM}$ use the letter $k$ for $\Delta$ in their form-factor expansions before their derivation of the sum rule. They treat both terms in the last line as non-trivial, but throw away the first one stating that it would account for the CoM motion of the wave-packet had one not worked with eigenstates of momentum but with wave-packets from the start.

\subsubsection{Derivation of Sum Rule}

But the spirit of the above is embodied in the following wave-packet treatment, using the wave-packet state mentioned above. It is defined again as a reminder for what follows:

$$
|\Phi\rangle=\int d^{3} p \phi(p)|p\rangle
$$

where $\phi$ is any weighting function. For example, it could be centered at $p=p_{o}$, $\phi(p)=\mathcal{N} \frac{1}{a} \exp -\frac{\left(p-p_{o}\right)^{2}}{a}$.

One can prepare the state $|\Phi\rangle$ any way one wants, of course. There are many paths one can take to string together states lying in a continuum of spin-momentum space. We can choose the general helicity state and thus always stay in an eigenstate of helicity as we vary the three-momentum. Or we can use the canonical state, i.e. start with a state of longitudinal $(z)$ spin projection in the rest frame and then boost in the desired momentum direction. For example, if we choose helicity eigenstates, then the spin projection, whether $p$ is longitudinal or transverse, will be along $p$ for the state $\mid p>$. For the canonical state, we will be neither in an eigenstate of helicity or spin in any direction. Whatever method we choose, we are forming a superposition of eigenstates of momentum, constructed according to some general prescription. So our formalism with wave packets is unaltered and the result stands generally. All the relevant information yielding the expectation value of $\vec{J}$ for the wave-packet and state prescription chosen is contained within the final expression. 
A small note is made on the general canonical wave-packet state. The typical state is a superposition of abstract cousins - states related by a continuous boost from a fixed rest-frame spin-projection- and are neither eigenstates of helicity or spin. We can ensure, should we desire, that these exhaust the Hilbert space by giving them a spin index, $s$, which can take on any of the allowed values of spin projection (e.g. up/down).

$|\Phi\rangle=\int d^{3} p \Sigma_{s} \phi_{s}(p)|p\rangle_{s}$

Because they are obtained by a boost from the rest frame with any allowed spin, they are most general. When we boost back to the rest frame, it is assured that they are allowed as well as restricted to have spin projecting in any of the possible directions, which means that they exhaust the spectrum.

To obtain the $z$-axis helicity state that most people work with when constructing spin sum rules, we just have to choose a wave packet that is peaked about the desired momentum, $p_{0}^{z}$. This will reduce the wave-packet state to the usual helicity state. Now, we will have an eigestate of spin and helicity, because as $k_{T} \rightarrow 0$ the states corresponding to this transverse momentum perturbation also do not contribute to the spinor, which is now purely in a helicity state. This is true regardless of where the $k_{T}$ states live in spin-momentum space, i.e. independent of whether it is a canonically constructed state or a helicity state. So, the expectation value of angular momentum in this particular $z$-helicity state can now be equated to the spin projection number (e.g. $\frac{1}{2}$ ) on the R.H.S.

The shape of the delta wave-packet we shall choose will be azimuthally symmetric (the reason for this will become clear shortly). In 2 dimensions, this would have been a Gaussian plotted $z$ vs $x$ and then rotated about the central axis, yielding azimuthal symmetry. In 3 dimensions, the narrow Gaussian wave-packet will actually have an extra symmetric dimension and there will be spherical symmetry in the wave-packet density about $p_{0}^{z}$. This is drawn below. The darkness of the contours represents density of wave-packet at that momentum.

One must also be careful here not to get a priori lost in the physical interpretation of a state or derivative that appears in the calculation below, but of the meaning of the original expression and whether we proceeded logically and robustly to the final one. If so, the final expression gives the same result as the initial and forms the basis 


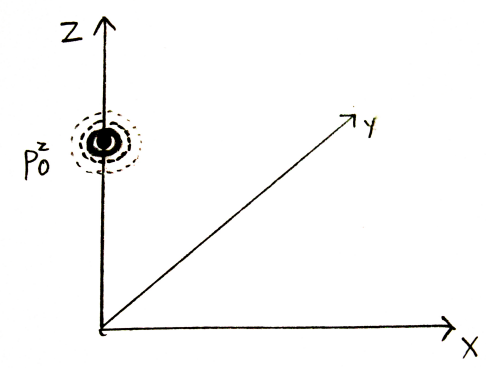

FiguRE 2.2: Gaussian wave-packet centered at $p_{0}^{z}$

for a sum rule. We will continually try to share phenomenological insights along the way.

Following is the wave-packet derivation of the sum rule - we were unaware at the time of doing this derivation that a similar approach has been taken by Shore and White [11], but the following is a physical state calculation and it specifically motivates the following observations. The normalization chosen is $<p^{\prime} \mid p>=2 p^{0} \delta\left(p^{\prime}-p\right)$. Note in the preceding that initial and final spin will not be the same. If the state has transverse momentum components, then we are no longer in an eigenstate of helicity or spin.

We insert the wave packets defined above to obtain

$$
\mathcal{M}^{\mu \nu \lambda}=\lim _{k \rightarrow 0} \int d^{3} x e^{i \mathbf{k} \cdot \mathbf{x}}\left\langle\Phi\left|M^{\mu \nu \lambda}\right| \Phi\right\rangle,
$$

and proceed analogously to section II. 


$$
\begin{aligned}
\mathcal{M}^{\mu \nu \lambda} & =\lim _{k \rightarrow 0} \int d^{3} x d^{3} p d^{3} p^{\prime} \phi(p) \phi^{*}\left(p^{\prime}\right) e^{i k x}\left\langle p^{\prime}\left|x^{\nu} T^{\mu \lambda}(x)-(\lambda \leftrightarrow \nu)\right| p\right\rangle \\
& =\lim _{k \rightarrow 0} \int d^{3} x d^{3} p d^{3} p^{\prime} \phi(p) \phi^{*}\left(p^{\prime}\right) x^{\nu} e^{i\left(k+p-p^{\prime}\right) \cdot x}\left\langle p^{\prime}\left|T^{\mu \lambda}(0)\right| p\right\rangle-(\lambda \leftrightarrow \nu) \\
& =\lim _{k \rightarrow 0} \int d^{3} x d^{3} p d^{3} p^{\prime} \phi(p) \phi^{*}\left(p^{\prime}\right)(-i) \frac{\partial}{\partial k_{\nu}} e^{i\left(k+p-p^{\prime}\right) \cdot x}\left\langle p^{\prime}\left|T^{\mu \lambda}(0)\right| p\right\rangle-(\lambda \leftrightarrow \nu) \\
& =\lim _{k \rightarrow 0} \int d^{3} p d^{3} p^{\prime} \phi(p) \phi^{*}\left(p^{\prime}\right)(-i) \frac{\partial}{\partial k_{\nu}} \delta^{3}\left(k+p-p^{\prime}\right)\left\langle p^{\prime}\left|T^{\mu \lambda}(0)\right| p\right\rangle-(\lambda \leftrightarrow \nu) \\
& =\lim _{k \rightarrow 0}(-i) \frac{\partial}{\partial k_{\nu}} \int d^{3} p d^{3} p^{\prime} \phi(p) \phi^{*}\left(p^{\prime}\right) \delta^{3}\left(k+p-p^{\prime}\right)\left\langle p^{\prime}\left|T^{\mu \lambda}(0)\right| p\right\rangle-(\lambda \leftrightarrow \nu) \\
& =\lim _{k \rightarrow 0}(-i) \frac{\partial}{\partial k_{\nu}} \int d^{3} p \phi(p+k) \phi(p)\left\langle p+k\left|T^{\mu \lambda}(0)\right| p\right\rangle-(\lambda \leftrightarrow \nu) \\
& =\lim _{k \rightarrow 0}(-i) \int d^{3} p \phi(p) \frac{\partial}{\partial k_{\nu}}\left[\phi^{*} i(p+k)\left\langle p+k\left|T^{\mu \lambda}(0)\right| p\right\rangle\right]-(\lambda \leftrightarrow \nu) \\
& =\lim _{k \rightarrow 0}(-i) \int d^{3} p \phi(p)\left\langle p+k\left|T^{\mu \lambda}(0)\right| p\right\rangle \frac{\partial}{\partial k_{\nu}} \phi(p+k)-(\lambda \leftrightarrow \nu) \\
& +(-i) \int d^{3} p \phi(p) \phi^{*}(p+k) \frac{\partial}{\partial k_{\nu}}\left\langle p+k\left|T^{\mu \lambda}(0)\right| p\right\rangle-(\lambda \leftrightarrow \nu) \\
& =\lim _{\mathbf{k} \rightarrow \mathbf{0}}(-\mathbf{i}) \int \mathbf{d}^{\mathbf{3}} \mathbf{p} \phi(\mathbf{p})\left\langle\mathbf{p}\left|\mathbf{T}^{\mu \lambda}(\mathbf{0})\right| \mathbf{p}\right\rangle \frac{\partial}{\partial \mathbf{k}_{\nu}} \phi^{*}(\mathbf{p}+\mathbf{k})-(\lambda \leftrightarrow \nu) \\
& +(-\mathbf{i}) \int \mathbf{d}^{3} \mathbf{p}|\phi(\mathbf{p})|^{\mathbf{2}} \frac{\partial}{\partial \mathbf{k}_{\nu}}\left\langle\mathbf{p}+\mathbf{k}\left|\mathbf{T}^{\mu \lambda}(\mathbf{0})\right| \mathbf{p}\right\rangle-(\lambda \leftrightarrow \nu)
\end{aligned}
$$

Note that we have neglected off-peak time-dependence here and done the calculation at some specific time.

\section{Comments}

1) The second term above is similar to the originally quoted sum rule in the literature. In fact it reduces to exactly that if the wave packet is taken to be a delta (square root of delta, really) peaked around the desired momentum $p_{0}$ :

$(-\mathbf{i})\left[\frac{\partial}{\partial \mathbf{k}_{\nu}}\left\langle\mathbf{p}_{\mathbf{0}}+\mathbf{k}\left|\mathbf{T}^{\mu \lambda}(\mathbf{0})\right| \mathbf{p}_{\mathbf{0}}\right\rangle-(\lambda \leftrightarrow \nu)\right]$.

The first term is perhaps what Jaffe calls the "center of mass" motion term of the wavepacket. This term disappears for us as pointed out below. So,

$$
\mathbf{M}^{\mu \nu \lambda}(\mathbf{x})=(-\mathbf{i})\left[\frac{\partial}{\partial \mathbf{k}_{\nu}}\left\langle\mathbf{p}_{\mathbf{0}}+\mathbf{k}\left|\mathbf{T}^{\mu \lambda}(\mathbf{0})\right| \mathbf{p}_{\mathbf{0}}\right\rangle-(\lambda \leftrightarrow \nu)\right]
$$


So when this result is applied to the spin-one hadron via GPDs [10], it yields:

$$
\mathbf{J}_{\mathbf{q}}=\frac{1}{2} \int \mathrm{d} \times \mathrm{xH}_{2}^{\mathrm{q}}(\mathrm{x}, \mathbf{0}, \mathbf{0})
$$

2) As Shore and White point out [11], the first term disappears when an azimuthally symmetric wave-packet is used. Similar to quantum mechanics, the one-particle state has no orbital angular momentum when its wave function has this property. So, this is the part that completely disappears for a one-particle noninteracting theory and is completely responsible for its orbital angular momentum. In the rest frame for such a theory and see that the first term gives no contribution at all (since the particle is at rest, it has no orbital angular momentum). One can extend this to say that this term contributes only to OAM and not to spin for any state, because, generally, if it had another part that was not an orbital contribution, it would have survived the azimuthal evaluation. This of course does not say anything about the spin phenonmenology of the second term.

This first term is not a consequence of using a wave-packet, but of using a non-azimuthallysymmetric wave-packet. An appropriately prepared momentum eigenstate will not have this contribution.

This is purely an OAM contribution since spin would not disappear for all general azimuthally symmetric states. Since this is valid for multiparticle states as well, the spatial wave-function symmetry is no longer necessary for this term to disappear. As long as the wave packet is azimuthally symmetric in total momentum space, this contribution disappears. Therefore, one can hypothesize that this is a pure OAM contribution that disappears with azimuthal momentum symmetry for any kind of state.

Also, is an interacting state with a fixed momentum unique? We can imagine degeneracy in many different free-particle states that give the same overall momentum eigenvalue. However, in the rest frame the proton has fixed spin.

3) Additionally, it would seem that the strict way to implement the sum rule is to change one of the kets' (here, the final ket) momentum in the transverse direction to obtain the sum rule and not just change the difference, $\Delta^{u}$, transversely. After all, the spinors are sandwiching the decomposition and only what is in between them is guaranteed to be a function of $\Delta^{2}$. So, the way one takes the derivative matters, because the way to vary the final ket transversely is not unique because it depends on the initial general state 
we use (canonical etc., see below).

4) What we did here is applicable equally to OAM as well, if $T_{C}$ is used instead of $T_{B}$. Each is related to AM/OAM in the following manner:

$$
M^{\mu \nu \lambda}=\int d^{3} x\left\langle p^{\prime}\left|x^{\nu} T^{\mu \lambda}(x)-(\lambda \leftrightarrow \nu)\right| p\right\rangle
$$

So, the inattentive or kind reader may ask: are total and orbital angular momentum the same? The answer, of course, is that only the total expectation value is preserved in the redefinition to the Belinfante form, not the density, which is weighted by and integrated over coordinates in position space. This is why, in the momentum sum rule, the role of the Belinfante EMT is not especially remarkable.

5) Please note that there is, as should be, origin dependence for angular momentum with smooth wave-packet states. This, and more with origin shift, is shown later. The dependence of origin here is reflected in the particular choice of space-point zero at which the EMT decomposition is done.

6) Additionally, one can use this formalism for different Gaussian wave packets, with some free parameter, to evaluate the angular momentum of the generic free-paramter state once a particular (e.g. longitudinal) case has been used to fix the sum rule and structure function coeffecients. This means we can ask (answer) the question, what is the expectation value of angular momentum of the generic wave-packet state?

7) Finally, let us note that $\phi(\mathbf{p}) \frac{\partial}{\partial \mathbf{k}_{\nu}} \phi(\mathbf{p}+\mathbf{k})\left\langle\mathbf{p}+\mathbf{k}\left|\mathbf{T}^{\mu \lambda}(\mathbf{0})\right| \mathbf{p s}\right\rangle$ is like the momentumspace density of angular momentum according to the form above.

\subsubsection{Obtaining a sum-rule with wave-packets}

As can be seen from the above, the wave-packet inherently enters the sum rule. There can be no sensible sum rule with just the eigenstates of momentum- so many standard properties like AM invariance under origin-shift for a state at rest, obtaining a finite expectation value for AM etc do not hold, just as in ordinary quantum mechanics. We 
discuss translation invariance below.

This wave-packet treatment nullifies all concerns regarding the change of spin as one changes momentum in the derivation of the sum rule. The relation obtained above is formally correct, and one can equate the RHS to $\frac{1}{2}$ if we use a nucleon wave-packet strongly peaked symmetrically about $p^{z}$, with longitudinal spin. The general form of the spinors is chosen canonically, i.e. such that when the momentum is purely along $z$, we will have an eigenstate of helicity and $\operatorname{spin}+\frac{1}{2}$ along $z$. The relative phases will be so minute (tend to zero if a delta function peaked at the desired momentum $p_{0}$ is used) that the expectation value will be unaffected.

\subsubsection{Translation-invariance for momentum and spin and translation- dependence for OAM}

For angular momentum, the ket phases from translation add up sensibly to show how an origin-shift affects the calculation and how it does not affect it when only spin is involved. Since spin is a pure dipole moment, we guess that a shift of origin in calculating the AM for a particle at rest (or seen in a longitudinally boosted frame) will not affect the result. Else, since wave-packets are off-peak in momentum, they are also not unbiased in position-space and hence a very large origin shift would affect the angular momentum. We do the following three origin-shift calculations in order: prove independence of origin for ordinary momentum, show dependence on origin for AM, and prove independence of origin for pure spin. Mathematical manipulation of derivatives in integrals involving delta functions below was possible since these are convergent quantities for smooth wave packets.

In all three cases, we shift the states by $a^{\nu}$ in the same coordinate system:

1) Expectation value of momentum: 


$$
\begin{aligned}
\mathcal{T}^{\mu \lambda} & \equiv \int d^{3} x d^{3} p d^{3} p^{\prime} \phi(p) \phi\left(p^{\prime}\right)\left\langle p^{\prime}\left|T^{\mu \lambda}(x-a)\right| p\right\rangle \\
& =\int d^{3} x d^{3} p d^{3} p^{\prime} \phi(p) \phi\left(p^{\prime}\right) e^{i\left(p-p^{\prime}\right) \cdot(x-a)}\left\langle p^{\prime}\left|T^{\mu \lambda}(0)\right| p\right\rangle \\
& =\int d^{3} p d^{3} p^{\prime} \phi(p) \phi\left(p^{\prime}\right) \delta^{3}\left(p-p^{\prime}\right)\left\langle p^{\prime}\left|T^{\mu \lambda}(0)\right| p\right\rangle e^{-i\left(p-p^{\prime}\right) \cdot a} \\
& =\int d^{3} p|\phi(p)|^{2}\left\langle p\left|T^{\mu \lambda}(0)\right| p\right\rangle \\
& =\int d^{3} p|\phi(p)|^{2}\left\langle p\left|T^{\mu \lambda}(0)\right| p\right\rangle \\
& =\int d^{3} x d^{3} p d^{3} p^{\prime} \phi(p) \phi\left(p^{\prime}\right)\left\langle p^{\prime}\left|T^{\mu \lambda}(x)\right| p\right\rangle
\end{aligned}
$$

2) Expectation value of AM:

$$
\begin{aligned}
\mathcal{M}^{\mu \nu \lambda} & =\lim _{k \rightarrow 0} \int d^{3} x d^{3} p d^{3} p^{\prime} \phi(p) \phi\left(p^{\prime}\right)\left\langle p^{\prime}\left|x^{\nu} T^{\mu \lambda}(x-a)-(\lambda \leftrightarrow \nu)\right| p\right\rangle \\
& =\lim _{k \rightarrow 0} \int d^{3} x d^{3} p d^{3} p^{\prime} \phi(p) \phi\left(p^{\prime}\right) x^{\nu} e^{i\left(k+p-p^{\prime}\right) \cdot(x-a)}\left\langle p^{\prime}\left|T^{\mu \lambda}(0)\right| p\right\rangle-(\lambda \leftrightarrow \nu) \\
& =\lim _{k \rightarrow 0} \int d^{3} x d^{3} p d^{3} p^{\prime} \phi(p) \phi\left(p^{\prime}\right)(-i) \frac{\partial}{\partial k_{\nu}} e^{i\left(k+p-p^{\prime}\right) \cdot x}\left\langle p^{\prime}\left|T^{\mu \lambda}(0)\right| p\right\rangle e^{-i\left(k+p-p^{\prime}\right) \cdot a}-(\lambda \leftrightarrow \nu) \\
& =\lim _{k \rightarrow 0}(-i) \frac{\partial}{\partial k_{\nu}} \int d^{3} p d^{3} p^{\prime} \phi(p) \phi\left(p^{\prime}\right) \delta^{3}\left(k+p-p^{\prime}\right) e^{-i\left(k+p-p^{\prime}\right) \cdot a}\left\langle p^{\prime}\left|T^{\mu \lambda}(0)\right| p\right\rangle \\
& -(-i) \int d^{3} p d^{3} p^{\prime} \phi(p) \phi\left(p^{\prime}\right) \delta^{3}\left(k+p-p^{\prime}\right) \frac{\partial}{\partial k_{\nu}} e^{-i\left(k+p-p^{\prime}\right) \cdot a}\left\langle p^{\prime}\left|T^{\mu \lambda}(0)\right| p\right\rangle-(\lambda \leftrightarrow \nu) \\
& =(-i) \lim _{k \rightarrow 0}\left[\frac{\partial}{\partial k_{\nu}} \int d^{3} p \phi(p) \phi(p+k)\left\langle p+k\left|T^{\mu \lambda}(0)\right| p\right\rangle\right. \\
& \left.-i a^{\nu} \int d^{3} p \phi(p) \phi(p+k)\left\langle p+k\left|T^{\mu \lambda}(0)\right| p\right\rangle\right]-(\lambda \leftrightarrow \nu) \\
& =(-i) \lim _{k \rightarrow 0} \frac{\partial}{\partial k_{\nu}} \int d^{3} p \phi(p) \phi(p+k)\left\langle p+k\left|T^{\mu \lambda}(0)\right| p\right\rangle \\
& -a^{\nu} \int d^{3} p|\phi(p)|^{2}\left\langle p\left|T^{\mu \lambda}(0)\right| p\right\rangle-(\lambda \leftrightarrow \nu)
\end{aligned}
$$

The first term is independent of the shift, whereas the second term contains it. The second term disappears in the rest frame or if the wave-packet $\phi(p)$ is azimuthally symmetric even up to a phase (e.g. uniformly small or zero transverse momentum), because it contains the expectation value of transverse momentum. The mathematical subtlety above is that the $k$-derivative does not commute with $\int d^{3} p^{\prime}$ because the latter will remove the $k$-dependence of the remaining exponential. Else one would erroneously conclude that OAM is independent of origin for wave-packet states! In the first wave-packet 
derivation above, the choice of origin is reflected in the fact that the decomposition is done at a particular origin, and would be different for different origins for smooth wavepackets. The phases here add up non-trivially to give origin-dependence.

3) Now, in the rest frame (where there is no OAM for non-composite states) and in states with azimuthal WP symmetry, one just has to observe that the second term above is zero.

$\int d^{3} p|\phi(p)|^{2}\left\langle p\left|T^{\mu \lambda}(0)\right| p\right\rangle-(\lambda \leftrightarrow \nu)=0$

This is a surprising result: even for composite multi-particle states, as long as the total momentum is zero, the total angular momentum is independent of origin! When we have partons, as long as the total momentum of the hadron is zero, we can treat it as a pure one-particle state (e.g. proton as Dirac fermion) with respect to spin invariance under origin-shift. This follows from the fact that since spin is a pure dipole moment, its expectation value should be independent of the origin used and is by itself equal to the total angular momentum in the rest frame.

Also note that there is no OAM in the longitudinal direction if there are no transverse momentum components, regardless of what origin is used.

\subsection{Gravitational Form Factors}

\subsubsection{The Connection with GPDs}

Let us consider $T^{\mu \nu}(x)$. The general expectation value is written

$\left\langle p^{\prime} \frac{i}{2}\left[\bar{\psi}(x) \gamma^{\mu} \overrightarrow{\partial_{\nu}} \psi(x)+\bar{\psi}(x) \gamma^{\mu} \overleftarrow{\partial_{\nu}} \psi(x)\right] p\right.$

This is decomposed by the authors for the deuteron [10] as follows at space-time point $x=0$ : 


$$
\begin{aligned}
& \left\langle p^{\prime}\left|T^{\mu \nu}\right| p\right\rangle=-\frac{1}{2} P^{\mu} P^{\nu}\left(\epsilon^{\prime *} \epsilon\right) \mathcal{G}_{1}(t) \\
- & \frac{1}{4} P^{\mu} P^{\nu} \frac{(\epsilon P)\left(\epsilon^{\prime *} P\right)}{M^{2}} \mathcal{G}_{2}(t)-\frac{1}{2}\left[\Delta^{\mu} \Delta^{\nu}-g^{\mu \nu} \Delta^{2}\right]\left(\epsilon^{\prime *} \epsilon\right) \\
\times & \mathcal{G}_{3}(t)-\frac{1}{4}\left[\Delta^{\mu} \Delta^{\nu}-g^{\mu \nu} \Delta^{2}\right] \frac{(\epsilon P)\left(\epsilon^{\prime *} P\right)}{M^{2}} \mathcal{G}_{4}(t) \\
+ & \frac{1}{4}\left[\left(\epsilon^{\prime * \mu}(\epsilon P)+\epsilon^{\mu}\left(\epsilon^{\prime *} P\right)\right) P^{\nu}+\mu \leftrightarrow \nu\right] \mathcal{G}_{5}(t) \\
+ & \frac{1}{4}\left[\left(\epsilon^{\prime * \mu}(\epsilon P)-\epsilon^{\mu}\left(\epsilon^{\prime *} P\right)\right) \Delta^{\nu}+\mu \leftrightarrow \nu\right. \\
+ & \left.2 g_{\mu \nu}(\epsilon P)\left(\epsilon^{\prime *} P\right)-\left(\epsilon^{\prime * \mu} \epsilon^{\nu}+\epsilon^{\prime * \nu} \epsilon^{\mu}\right) \Delta^{2}\right] \mathcal{G}_{6}(t) \\
+ & \frac{1}{2}\left[\epsilon^{* \prime \mu} \epsilon^{\nu}+\epsilon^{\prime * \nu} \epsilon^{\mu}\right] \mathcal{G}_{7}(t)+g^{\mu \nu}\left(\epsilon^{\prime *} \epsilon\right) M^{2} \mathcal{G}_{8}(t)
\end{aligned}
$$

If the two defining matrix elements of (a component of) the EMT at $x=0$ and the proton current were shown to be the same, then the analysis becomes simple. Since the hadron current is decomposed in Lorentz structures just like the hadron EMT is decomposed (with the latter's first and second indices identified with the "+" component [4]), their coefficients must also be equal term by term. These coefficients of course do not depend on the light-cone coordinate in question, as they are functions of the invariant $\Delta^{2}$. So, they are very generally equal to each other correspondingly. This is our third step of the sum rule derivation and application quoted above and here the hadron state used does not enter the picture. It does enter when the sum rule is formulated in the second step, i.e. when the EMT form factors are related to a number, which requires sandiwching of $J^{i}$ in between, for example, helicity eigenstates whose $z$-spin projection is known and is put on the RHS (and $i$ is here taken to be 3 ). This, then, puts a constraint on the second moment of the GPDs.

It is clear that if one wishes to formulate a longitudinal-spin sum rule one must equate the matrix element of $J$ to $\frac{1}{2}$, the longitudinal spin projection, and choose helicity states for the analysis. For this, $J^{z}$ must be chosen. That is: $\frac{\partial}{\partial k_{1}}\left\langle p_{0}+k\left|T^{02}(0)\right| p_{0}\right\rangle-(1 \leftrightarrow 2)=\frac{1}{2}$, assuming the helicity spinors are inserted above in the decomposition for $T^{\mu \nu}$ as given in [2]. Note that it is not the case, as is commonly believed, that one can just change the matrix element by $\Delta=p^{\prime}-p$, to obtain the sum rule. One must vary the ket as above in taking the $k$-derivative because the matrix element is not simply a function of $\Delta$.

$$
A_{q}(0)+A_{g}(0)=1 \frac{1}{2}\left(A_{q}(0)+A_{g}(0)+p^{+}\left(B_{q}(0)+B_{g}(0)\right)\right)=\frac{1}{2} \text { (if longitudinal used) }
$$


So, $B_{q}(0)+B_{g}(0)=0$. This ensures that the quark and gluon contributions do not individually diverge!

Next, let us relate the gravitational form factors from our decomposition to the spin-1 GPDs. We know that

$$
\begin{aligned}
& \int \frac{d \kappa}{2 \pi} e^{i x P^{+} \kappa}<p^{\prime}, \lambda^{\prime}|\bar{\psi}(-k n) \gamma \cdot n \psi(k n)| p^{\prime}, \lambda^{\prime}>=\epsilon^{\prime *} . \epsilon H_{1}+\frac{\epsilon^{\prime *} . P \epsilon \cdot n+\epsilon \cdot P \epsilon^{\prime *} . n}{P . n} H_{2} \\
& -\epsilon^{\prime *} . P \epsilon_{1} . P \frac{H_{3}}{2 M^{2}}+\frac{\epsilon^{\prime *} . P \epsilon . n-\epsilon \cdot P \epsilon^{\prime *} . n}{P . n} H_{4}+\left(\frac{4 M^{2} \epsilon^{\prime *} . n \epsilon \cdot n}{(P . n)^{2}}+\epsilon^{\prime *} . \epsilon \frac{1}{3}\right) H_{5}
\end{aligned}
$$

Using the above results, we see the following relations right away after equating the $T^{++}$ component to the above correlator $\left[\gamma^{+}\right]$:

$$
\begin{array}{r}
G_{1}+\xi^{2} G_{3}=\int d x\left(2 H_{1}(x) x-\frac{2}{3} H_{5}(x) x\right) \\
G_{2}+\xi^{2} G_{4}=\int d x 2 H_{3}(x) x \\
G_{5}=\int d x 2 H_{2}(x) x \\
\xi G_{6}=-4 \int d x H_{4}(x) x \\
-\frac{\Delta^{2}}{8 m^{2}} G_{6}+\frac{1}{2} G_{7}=\int d x H_{5}(x) x
\end{array}
$$

\subsubsection{Checking Individual Terms for Conservation}

Now we ask, which are the terms that could be non-conserving in the decomposition? But before that let us remind ourselves thoroughly what we mean by conservation here. In other words, let us explore a bit what the meaning of conserved and non-conserved terms in $T^{\mu \nu}$ is. We know that the momentum current $\left\langle T^{\mu \nu}(x)\right\rangle$ is conserved overall for any state, i.e. $\partial_{\mu}\left\langle T^{\mu \nu}(x)\right\rangle=0$. In a momentum eigenstate $T^{\mu \nu}(x)_{q, g}$ is also individually conserved. We can see this by translating the operator to $x=0$ :

$\partial_{\mu}\left\langle p^{\prime} \mid T^{\mu \nu}(x) p\right\rangle=\left(p-p^{\prime}\right)^{\mu} e^{i\left(p-p^{\prime}\right) \cdot x}\left\langle\mid T^{\mu \nu}(0)\right\rangle \rightarrow 0$ 
when $p \rightarrow p^{\prime}$. Thus, we see that in a momentum eigenstate, any current is conserved! This is of course another hint that observables should not be thought of seriously in such a treatment. Moreover, we can say that each form factor term is a conserving term since none of the terms contribute a finite value to the four-divergence. If we evaluate the operator off-forward, however, then the translation phases do not cancel. In a physical state, this will be due to the presence of off-forward cross-terms, $\left\langle p^{\prime}\left|T^{\mu \nu}(x)\right| p\right\rangle$, in evaluating the total expectation value from wave-packet integrals in the momentum basis - thus ensuring that not all operator currents are generally conserved. This is the real use of the off-forward check on current conservation. Thus, the individual contributions $T_{q, g}^{\mu \nu}$ have no reason to be separately conserved - there will be at least one non-conserving term in each. However, a quick calculation involving an arbitrary wavepacket shows that $T^{\mu \nu}$ is conserved in an off-forward sense since it must be conserved in any state (else it would be a non-zero function which has a Fourier Transform of zero).

So now in the same but generalized spirit as forward conservation, we speak of off-forward conservation. If a vector operator is not conserved in an off-forward matrix element of momentum eigenstates, then it won't be conserved for a physical wave-packet state. And if it is conserved in an off-forward sense for all momentum eigenstates, then it will be conserved for all physical states in the appropriate Hilbert space of the theory (which can be thought as composed of such basis momentum states). This motivates off-forward conservation physically rather than their just being a mathematical book-keeping variant of usual current conservation. Of course, for a symmetric energy-momentum tensor, the current index can be either.

Let us check the $\epsilon^{\prime * v} \epsilon^{u}+\epsilon^{\prime * u} \epsilon^{v}$ term, whose coefficient is our $G_{7}\left(\Delta^{2}\right)$ :

$$
\begin{aligned}
& \partial_{u}\left\langle p^{\prime}\left|T^{u v}(x)\right| p\right\rangle \\
= & \partial_{u}\left(e^{i \Delta \cdot x}\left\langle p^{\prime}\left|T^{u v}(0)\right| p\right\rangle\right. \\
= & i \Delta_{u} e^{i \Delta \cdot x}\left\langle p^{\prime}\left|T^{u v}(0)\right| p\right\rangle \\
= & i \Delta_{u} e^{i \Delta \cdot x}\left[G_{1}\left[P^{u}, P^{v}\right] \epsilon^{\prime *} \cdot \epsilon+\ldots G_{7}\left(\epsilon^{\prime * v} \epsilon^{u}+\epsilon^{\prime * u} \epsilon^{v}\right)\right] \\
= & 0+i\left(p_{u}^{\prime} \epsilon^{\prime * v} \epsilon^{u}-p_{u} \epsilon^{* * u} \epsilon^{v}\right)
\end{aligned}
$$

This $G_{7}$ term is manifestly not conserved for all components, but it is clearly conserved for longitudinal spin. 
It is conserved for longitudinal spin, not in general. It is not conserved in the same sense as the second Mellin moment of $H_{5}$, so there is no problem having it in the "conserving" part of the decomposition. The Mellin moment's being "conserving" is related to the fact that each GPD term defining the total quark electromagnetic current has to be conserved. This one goes to zero in fact, so is clearly conserved when integrated with respect to momentum fraction, which one does to obtain current at $x=0$.

Let us address gauge-invariance briefly, vis-a-vis conserved quantities. Gauge-invariance of an operator does not mean its conservation as 4-current. If the Lagrangian of the theory is gauge-invariant (i.e. the theory/EOMs are gauge-invariant), then $\partial_{\mu} j^{\mu}=0$ for the current of the theory. In QED, the electromagnetic current is conserved, and this conservation is promoted to quantum conservation in the sense of the expectation value of the divergence of this operator being zero. Thus, the theory (and so, presumably, all associated observable operators) being gauge-invariant implies the conservation of a particular current. The gauge-invariance of operators does not have a separate consequence for a different current, but is itself a consequence of the gauge-invariance of the theory. Observable operators are usually gauge-invariant and that comes from a different yet appealing classical symmetry, but the current corresponding to a particular observable need not be conserved. What if an observable operator were not a vector? Then which quantity related to it would be conserved? Similarly, even though the $G_{7}$ term is not manifestly conserved, it is still allowed to be gauge-invariant (especially because it is connected to a GPD!). 


\section{Chapter 3}

\section{Deuteron}

\subsection{Background}

The basics have been given in the introduction. So, now we proceed with the structure functions of the deuteron. The deuteron current can be decomposed into 3 form factors, $G_{1}, G_{2}$ and $G_{3}[12]$ :

$$
\begin{aligned}
& \int \frac{d \kappa}{2 \pi} e^{i x P^{+} \kappa}<p^{\prime}, \lambda^{\prime}|\bar{\psi}(-k n) \gamma \cdot n \psi(k n)| p^{\prime}, \lambda^{\prime}> \\
& =\epsilon^{\prime *} . \epsilon G_{1}+\frac{\epsilon^{\prime *} \cdot P \epsilon \cdot n+\epsilon \cdot P \epsilon^{\prime *} \cdot n}{P \cdot n} G_{2} \\
& -\epsilon^{\prime *} . P \epsilon_{1} \cdot P \frac{G_{3}}{2 M^{2}}
\end{aligned}
$$

The form factors $G_{i}(t)=\int H_{i}(x, t, \zeta) d x$ for $i=1,2,3$. The other two integrate to zero, i.e. $\int H_{4}(x, t, \zeta) d x=\int H_{5}(x, t, \zeta) d x=0$.

These are the analogs of the Dirac and Pauli form factors for the nucleon, and combine to give the charge, magnetic (dipole) and electric quadrupole form factors $\left(G_{C}, G_{M}\right.$ and $G_{Q}$ respectively) in the following way: 


$$
\begin{aligned}
& G_{C}(t)=G_{1}(t)+\frac{2}{3} \eta G_{Q} \\
& G_{M}(t)=G_{2}(t) \\
& G_{Q}(t)=G_{1}(t)-G_{2}(t)+(1+\eta) G_{3}(t)
\end{aligned}
$$

where $\eta=\frac{t}{2 m^{2}}$ and $m$ is the mass of the deuteron.

The quadrupole moment appears since the deuteron is not spherically symmetric like the proton is. A spherically symmetric particle's potential does not generate an electric quadrupole moment.

\subsection{Energy-Momentum Tensor Decomposition}

The EMT for the deuteron at a particular space-time point (0) can be decomposed in the following way, for the quark and the gluon individually as before (here, $q, g$ indices have been suppressed below) using the $J^{P C}$ quantum number rules from the OPE of the spin-one matrix element:

$$
\begin{aligned}
& \left\langle p^{\prime}\left|T^{\mu \nu}\right| p\right\rangle=-\frac{1}{2} P^{\mu} P^{\nu}\left(\epsilon^{\prime *} \epsilon\right) \mathcal{G}_{1}(t) \\
- & \frac{1}{4} P^{\mu} P^{\nu} \frac{(\epsilon P)\left(\epsilon^{\prime *} P\right)}{M^{2}} \mathcal{G}_{2}(t)-\frac{1}{2}\left[\Delta^{\mu} \Delta^{\nu}-g^{\mu \nu} \Delta^{2}\right]\left(\epsilon^{\prime *} \epsilon\right) \\
\times & \mathcal{G}_{3}(t)-\frac{1}{4}\left[\Delta^{\mu} \Delta^{\nu}-g^{\mu \nu} \Delta^{2}\right] \frac{(\epsilon P)\left(\epsilon^{\prime *} P\right)}{M^{2}} \mathcal{G}_{4}(t) \\
+ & \frac{1}{4}\left[\left(\epsilon^{\prime * \mu}(\epsilon P)+\epsilon^{\mu}\left(\epsilon^{\prime *} P\right)\right) P^{\nu}+\mu \leftrightarrow \nu\right] \mathcal{G}_{5}(t) \\
+ & \frac{1}{4}\left[\left(\epsilon^{\prime * \mu}(\epsilon P)-\epsilon^{\mu}\left(\epsilon^{\prime *} P\right)\right) \Delta^{\nu}+\mu \leftrightarrow \nu\right. \\
+ & \left.2 g_{\mu \nu}(\epsilon P)\left(\epsilon^{\prime *} P\right)-\left(\epsilon^{\prime * \mu} \epsilon^{\nu}+\epsilon^{\prime * \nu} \epsilon^{\mu}\right) \Delta^{2}\right] \mathcal{G}_{6}(t) \\
+ & \frac{1}{2}\left[\epsilon^{* \prime \mu} \epsilon^{\nu}+\epsilon^{\prime * \nu} \epsilon^{\mu}\right] \mathcal{G}_{7}(t)+g^{\mu \nu}\left(\epsilon^{\prime *}\right)
\end{aligned}
$$

There are seven conserving terms (i.e. they do not violate current conservation even if the quark and gluon parts of the EMT are taken separately, in all aspects for the 
components in question at the very least) up to twist 2 and one non-conserving term, $G_{8}$, is inserted analogous to Ji's $\bar{C}$ term [2].

\subsubsection{Trace Anomaly}

It is sometimes mentioned that the OPE should yield all traceless terms. Let us research this a little bit. What is interesting to note is that the dilatation current, $D^{\mu}=T^{\mu \nu} x_{\nu}$, must be overall non-conserved due to the QCD trace anomaly, i.e. $\partial_{\mu} D^{\mu}=T_{\mu}^{\mu}$, which is not zero. However, this does not mean that it cannot be conserved for particular terms. Some algebra yields:

$\partial_{\mu} D^{\mu}=\partial_{\mu} T^{\mu \nu} x_{\nu}+T_{\mu}^{\mu}$

This means that at least some individual terms will have to violate dilatation current conservation. There will be some terms that will conserve momentum and be traceless (and hence conserve dilatation current by the above equation), but there will be some that conserve momentum but are not traceless. The non-conserving term $G_{8}$ can have a trace but it will not give rise to the trace anomaly, because $G_{8, q}=-G_{8, g}$. This means that at least one of the individual-momentum (quark or gluon)-conserving terms will not conserve dilatation and will have a trace. Thus, the OPE cannot yield all traceless terms. This also shows that there is a requirement that some terms be individual-momentumconserving, else we would not recover the trace anomaly.

\subsection{Spin One Sum Rule}

We now derive a sum rule for the total quark angular momentum of a spin-one hadronic system within a gauge invariant decomposition of the hadron's spin. We also show that the total angular momentum can be measured through deeply virtual Compton scattering experiments using transversely polarized deuterons. In the following, some background information will be repeated in this section more extensively for proper context, not for redundancy purposes. The work that follows in this section was written collaboratively [10]. 
A crucial, outstanding question in $\mathrm{QCD}$ is the proton spin puzzle. A number of experiments performed since the '80s, including the most recent HERMES, Jefferson Lab and Compass measurements, have confirmed that only about $30 \%$ of the proton spin is accounted by quarks, and that the quark contribution is dominated by the valence component (see review in [21]). Current efforts, both in theory and experiment, are therefore directed towards determining the contributions of the Orbital Angular Momentum (OAM) of the quarks, as well as of the spin and OAM of the gluons. Sum rules were derived that relate the Energy Momentum Tensor's (EMT) form factors to the nucleon angular momentum [2, 5]. ${ }^{1}$ In [5], starting from the classical/canonical form of the EMT, it is possible to identify the four contributions from the quark and gluon OAM and spin components. Of these only the quark and gluon spin terms appear among the observables for hard scattering processes. On the other side, the result derived in [2], uses the symmetric, Belinfante form of the EMT and leads to different definitions of the angular momentum components, $J_{q}=L_{q}+\Delta \Sigma$, and $J_{g}$. These can, in principle, be measured through Deeply Virtual Compton Scattering (DVCS) (see also [19]). However, the interpretation of these components in terms of unintegrated parton angular momentum density distributions is not straightforward. The values of the observables will therefore differ in the two approaches [3].

Motivated by the challenge of the spin puzzle on one side, and by the feasibility of DVCS type experiments, we decided to investigate the angular momentum sum rules for hadronic systems of different spin which are provided, in practice, by nuclear targets. In this contribution we present a sum rule for the total angular momentum in a spin one nucleus, the deuteron. The sum rule is of particular relevance because it involves only one Generalized Parton Distribution (GPD), namely

$$
J_{q}=\frac{1}{2} \int d x x H_{2}^{q}(x, 0,0) .
$$

$H_{2}^{q}(x, \xi, t)$ 's first moment is equal to the deuteron magnetic form factor $G_{2}(t) \equiv G_{M}(t)$ [14]. This expression can be compared to the nucleon sum rule [2],

$$
J_{q}=\frac{1}{2} \int d x x\left[H_{q}(x, 0,0)+E_{q}(x, 0,0)\right]
$$

where the first moment of the GPD sum $H_{q}(x, \xi, t)+E_{q}(x, \xi, t)$ is the nucleon magnetic form factor, $F_{1}(t)+F_{2}(t) \equiv G_{M}(t)$. Similar to the proton GPD $E, H_{2}$ does not have a forward partonic limit.

\footnotetext{
${ }^{1}$ Alternative procedures to obtain explicit gauge invariant operators for spin and orbital angular momentum of quarks and gluons were given in $[24,25]$. Their discussion is beyond the scope of this paper, see however [7].
} 
In what follows we outline the fundamental steps of the derivation. We start from the expression for angular momentum in QCD,

$$
J^{i}=\frac{1}{2} \epsilon^{i j k} \int d^{3} x M^{0 j k}
$$

where the tensor $M^{0 i j}$ is the angular momentum density given in terms of the symmetric, gauge-invariant, and conserved (Belinfante) EMT as $M^{\alpha \mu \nu}=T^{\alpha \nu} x^{\mu}-T^{\alpha \mu} x^{\nu}$. Notice that $T^{\mu \nu}$ has separate gauge invariant contributions from quarks and gluons [2], along with their interaction through the gauge-covariant derivative.

$$
\begin{aligned}
T^{\mu \nu} & =T_{q}^{\mu \nu}+T_{g}^{\mu \nu}=\frac{1}{2}\left[\bar{\psi} \gamma^{(\mu} i \overrightarrow{\left.D^{\nu}\right)} \psi+\bar{\psi} \gamma^{(\mu} i \overleftarrow{D^{\nu)}} \psi\right] \\
& +\frac{1}{4} g^{\mu \nu} F^{2}-F^{\mu \alpha} F_{\alpha}^{\nu}
\end{aligned}
$$

The connection of GPDs to the angular momentum becomes apparent by first writing down the matrix element of $T_{q, g}^{\mu \nu}$ for a spin-one system in terms of gravitational form factors as,

$$
\begin{aligned}
& \left\langle p^{\prime}\left|T^{\mu \nu}\right| p\right\rangle=-\frac{1}{2} P^{\mu} P^{\nu}\left(\epsilon^{\prime *} \epsilon\right) \mathcal{G}_{1}(t) \\
- & \frac{1}{4} P^{\mu} P^{\nu} \frac{(\epsilon P)\left(\epsilon^{\prime *} P\right)}{M^{2}} \mathcal{G}_{2}(t)-\frac{1}{2}\left[\Delta^{\mu} \Delta^{\nu}-g^{\mu \nu} \Delta^{2}\right]\left(\epsilon^{\prime *} \epsilon\right) \\
\times & \mathcal{G}_{3}(t)-\frac{1}{4}\left[\Delta^{\mu} \Delta^{\nu}-g^{\mu \nu} \Delta^{2}\right] \frac{(\epsilon P)\left(\epsilon^{\prime *} P\right)}{M^{2}} \mathcal{G}_{4}(t) \\
+ & \frac{1}{4}\left[\left(\epsilon^{\prime * \mu}(\epsilon P)+\epsilon^{\mu}\left(\epsilon^{\prime *} P\right)\right) P^{\nu}+\mu \leftrightarrow \nu\right] \mathcal{G}_{5}(t) \\
+ & \frac{1}{4}\left[\left(\epsilon^{\prime * \mu}(\epsilon P)-\epsilon^{\mu}\left(\epsilon^{\prime *} P\right)\right) \Delta^{\nu}+\mu \leftrightarrow \nu\right. \\
+ & \left.2 g_{\mu \nu}(\epsilon P)\left(\epsilon^{\prime *} P\right)-\left(\epsilon^{\prime * \mu} \epsilon^{\nu}+\epsilon^{\prime * \nu} \epsilon^{\mu}\right) \Delta^{2}\right] \mathcal{G}_{6}(t) \\
+ & \frac{1}{2}\left[\epsilon^{* \prime \mu} \epsilon^{\nu}+\epsilon^{\prime * \nu} \epsilon^{\mu}\right] \mathcal{G}_{7}(t)+g^{\mu \nu}\left(\epsilon^{\prime *} \epsilon\right) M^{2} \mathcal{G}_{8}(t)
\end{aligned}
$$

where $t=\Delta^{2}, P=p+p^{\prime}$ and $\Delta=p^{\prime}-p$, and $\epsilon, \epsilon^{\prime}$ are the polarization vectors of the deuteron in the initial and final helicity states, respectively. There are seven conserved independent form factors, $\mathcal{G}_{i}(t), i=1,7$, and an additional non conserved term, $g^{\mu \nu}\left(\epsilon^{\prime *} \epsilon\right) M^{2} \mathcal{G}_{8}(t)$. In analogy with the nucleon case [30, 31], the enumeration of the independent deuteron EMT form factors, as well its Lorentz structure, was obtained using the partial wave formalism and crossing symmetry (details on our method for counting the form factors are presented in [32] (nucleon) and in an upcoming paper [33] (deuteron)).

Following a point raised in Ref.[19], we carefully used a wave-packet approach to derive the relation between $J^{z}$ and the EMT [5]. From Eq.(3.4), and using Eq.(3.6) for a spin 
one system,

$$
J_{q, g}^{z}=\frac{1}{2} \mathcal{G}_{5}(0)
$$

One can now connect the gravitational form factors with the coefficients of the correlator for (unpolarized) DVCS. For a spin one system one can write this in terms of five unpolarized GPDs (from the Lorentz symmetric part of the hadronic tensor) [14],

$$
\begin{aligned}
& \int \frac{d \kappa}{2 \pi} e^{i x \kappa P . n}\left\langle p^{\prime}, \lambda^{\prime}|\bar{\psi}(-\kappa n) \gamma \cdot n \psi(\kappa n)| p, \lambda\right\rangle \\
& =-\left(\epsilon^{\prime *} . \epsilon\right) H_{1}+\frac{(\epsilon . n)\left(\epsilon^{\prime *} . P\right)+\left(\epsilon^{\prime *} . n\right)(\epsilon . P)}{P . n} H_{2} \\
& -\frac{(\epsilon . P)\left(\epsilon^{\prime *} . P\right)}{2 M^{2}} H_{3}+\frac{(\epsilon . n)\left(\epsilon^{\prime *} \cdot P\right)-\left(\epsilon^{\prime *} . n\right)(\epsilon . P)}{P . n} H_{4} \\
& +\left\{4 M^{2} \frac{(\epsilon . n)\left(\epsilon^{\prime *} . n\right)}{(P . n)^{2}}+\frac{1}{3}\left(\epsilon^{\prime *} . \epsilon\right)\right\} H_{5}
\end{aligned}
$$

where $n$ is a light-like vector. It follows that by expanding the matrix element on the left hand side of Eq.(3.8) and taking the second moment with respect to $x$ one can find the following relation between the second moments of the GPDs $H_{i}$ and the form factors $\mathcal{G}_{i}$

$$
\begin{aligned}
& 2 \int d x x\left[H_{1}(x, \xi, t)-\frac{1}{3} H_{5}(x, \xi, t)\right]=\mathcal{G}_{1}(t)+\xi^{2} \mathcal{G}_{3}(t) \\
& 2 \int d x x H_{2}(x, \xi, t)=\mathcal{G}_{5}(t) \\
& 2 \int d x x H_{3}(x, \xi, t)=\mathcal{G}_{2}(t)+\xi^{2} \mathcal{G}_{4}(t) \\
& -4 \int d x x H_{4}(x, \xi, t)=\xi \mathcal{G}_{6}(t) \\
& \int d x x H_{5}(x, \xi, t)=-\frac{t}{8 M_{D}^{2}} \mathcal{G}_{6}(t)+\frac{1}{2} \mathcal{G}_{7}(t)
\end{aligned}
$$

For $t=0$ then one finds the sum rule relation between the deuteron GPD $H_{2}$, and the angular momentum $J_{q, g}$, shown in Eq.(3.2),

$$
J_{q, g}=\frac{1}{2} \int d x x H_{2}^{q, g}(x, 0,0) .
$$

This sum rule, which was derived following the same steps as for the spin $1 / 2$ case, is both the main result and the starting point of our paper. We now ask the questions: i) what is the parton content of $\mathrm{H}_{2}$, and $i$ ) can $\mathrm{H}_{2}$ be extracted from experiment with sufficient accuracy? In order to explain the partonic sharing of angular momentum in the deuteron we start from a picture in terms of bound nucleons. Eq.(3.8) can be written in terms of "quark-nucleus" helicity amplitudes that depend on $\xi, t$ and $Q^{2}$ while implicitly 


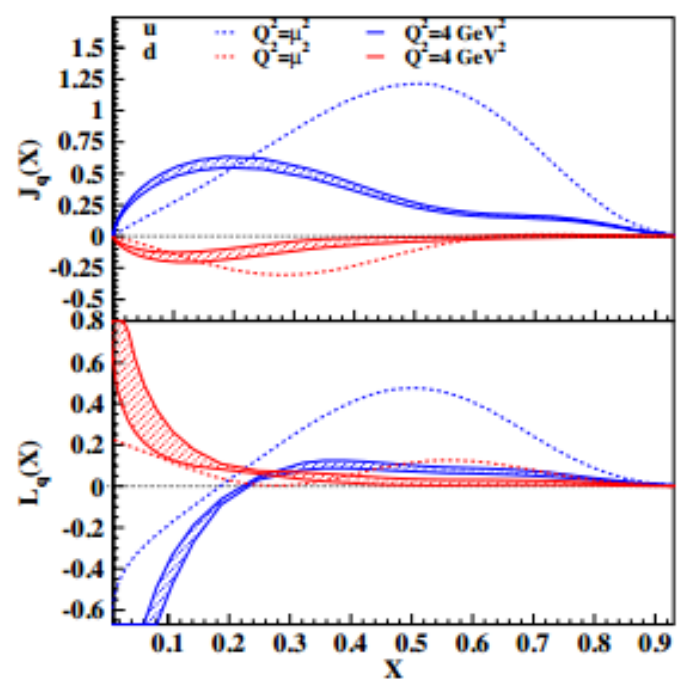

FIgURE 3.1: (color online) Upper panel: Total angular momentum density distributions, $J_{q}, q=u, d$, calculated using the GPD parametrization of Ref.[6]. Theoretical error bands are included. Lower panel: Orbital angular momentum density distributions, $L_{q}, q=u, d$, obtained from Eq.(3.19), using the parametrizations from $[6]\left(J_{q}\right)$ and [39] $(\Delta q)$. In both panels the dashed lines correspond to the scale $\mu^{2} \approx 0.1 \mathrm{GeV}^{2}$ where spectator models are evaluated [3]; the full lines from our fit results are calculated at $Q^{2}=4 \mathrm{GeV}^{2}$.

convoluting over the unobserved quark and nucleon momenta,

$$
C_{\Lambda^{\prime} \lambda_{q}^{\prime}, \Lambda \lambda_{q}}=\sum_{\lambda_{N}, \lambda_{N}^{\prime}} B_{\Lambda^{\prime} \lambda_{N}^{\prime}, \Lambda \lambda_{N}} \otimes A_{\lambda_{N}^{\prime} \lambda_{q}^{\prime}, \lambda_{N} \lambda_{q}}
$$

where $A_{\lambda_{N}^{\prime} \lambda_{q}^{\prime} ; \lambda_{N}, \lambda_{q}}$ and $B_{\lambda^{\prime}, \lambda_{N}^{\prime} ; \lambda, \lambda_{N}}$, are the quark-nucleon [6], and nucleon-deuteron helicity amplitudes, respectively, $\Lambda, \lambda_{N}, \lambda_{q}$, being the deuteron, nucleon, and quark helicities. $H_{2}$ can be explicitly evaluated from Eq.(3.15) using the convolution formalism that was developed in [35], taking care of the angular structure for the deuteron [13]. For $H_{2}(x, 0,0)=H_{2}$, only the $\left\{\Lambda^{\prime}, \Lambda\right\} \equiv\{1,1\},\{0,1\}$ deuteron helicity components contribute [13, 33],

$$
\begin{aligned}
& H_{2}=2 \sum_{\lambda_{q}}\left(C_{1 \lambda_{q}, 1 \lambda_{q}}-\frac{1}{\sqrt{2 \tau_{D}}} C_{1 \lambda_{q}, 0 \lambda_{q}}\right) \\
& \approx \int_{0}^{M_{D} / M} d z f^{\mathbf{1 , 1}}(z) H_{N}(x / z, 0,0)+f^{\mathbf{0 , 1}}(z) E_{N}(x / z, 0,0),
\end{aligned}
$$

where $H_{N}=H_{u}+H_{d}, E_{N}=E_{u}+E_{d}$, are the isoscalar nucleon GPDs, the kinematical variables, $x=k^{+} /\left(P_{D}^{+} / 2\right), z=p^{+} /\left(P_{D}^{+} / 2\right), p=|\mathbf{p}|, \tau_{D}=\left(t_{0}-t\right) / 2 M_{D}^{2}$, with $t_{0}=$ $-4 \xi^{2} M_{D}^{2} /\left(1-\xi^{2}\right)$, involve the quark, nucleon and deuteron four-momenta, $k_{\mu}, p_{\mu}$, and 
$P_{D, \mu}$, respectively,

$$
\begin{gathered}
f^{\mathbf{1}, \mathbf{1}}(z)=2 \pi M \int_{p_{\min }(z)}^{\infty} d p p \sum_{\lambda} \chi_{\mathbf{1}}^{* \lambda_{N_{1}}^{\prime} \lambda_{N_{2}}}(z, p) \chi_{\mathbf{1}}^{\lambda_{N_{1}} \lambda_{N_{2}}}(z, p) \\
f^{\mathbf{0 , 1}}(z)=4 \pi M \int_{p_{\min }(z)}^{\infty} d p p \sum_{\lambda} \chi_{\mathbf{0}}^{* \lambda_{N_{1}}^{\prime} \lambda_{N_{2}}}(z, p) \chi_{\mathbf{1}}^{\lambda_{N_{1}} \lambda_{N_{2}}}(z, p) .
\end{gathered}
$$

where $\lambda_{N_{1}}\left(\lambda_{N_{1}}^{\prime}\right)$ are the initial (returning) nucleons' helicities, $\lambda_{N_{2}}$ is the spectator nucleon one, the sum index is $\lambda=\left\{\lambda_{N_{1}}, \lambda_{N_{1}}^{\prime}, \lambda_{N_{2}}\right\} ; \chi_{\Lambda}^{\lambda_{N_{1}}, \lambda_{N_{2}}}(z, p)$ is the deuteron wave function [36, 37],

$$
\begin{aligned}
\chi_{\Lambda}^{\lambda_{N_{1}}, \lambda_{N_{2}}}(z, p) & =\mathcal{N} \sum_{L, m_{L}, m_{S}}\left(\begin{array}{ccc}
j_{1} & j_{2} & 1 \\
\lambda_{N_{1}} & \lambda_{N_{2}} & m_{S}
\end{array}\right)\left(\begin{array}{ccc}
L & S & J \\
m_{L} & m_{S} & \Lambda
\end{array}\right) \\
& \times Y_{L m_{L}}\left(\frac{\mathbf{p}}{p}\right) u_{L}(p) .
\end{aligned}
$$

In Eq.(3.18), $j_{1}=j_{2}=1 / 2, S=J=1 ; Y_{L m_{L}}$ depends on $\cos \theta=[M(1-z)-$ $E] / p, M$ being the nucleon mass and $E$ the deuteron's binding energy, consistently with the formalism for describing deep inelastic processes from nuclear targets [38] in the approximation where the quarks' $k_{\perp}$ dependence is trivially integrated over, and no off-shell effects are considered [35].

Our results are shown in Figures 3.1 and 3.2. In Fig.3.1 we present the proton $u$ and $d$ quarks components of both the total angular momentum density (upper panel), and the orbital angular momentum density (lower panel),

$$
L_{q}(x)=J_{q}(x)-\frac{1}{2} \Delta q(x),
$$

$\Delta q(x)$ being the quark polarized density, and $J_{q}(x)$ being the integrand in Eq.(3.3). Both the unpolarized and polarized $u$ and $d$ quarks GPDs used in the calculation are from the parametrization of Ref.[6]. The importance of perturbative QCD evolution is evident from the comparison of results at an initial low scale used e.g. in spectator models, $Q^{2}=\mu^{2} \approx 0.1 \mathrm{GeV}^{2}$, and evolved to $Q^{2}=4 \mathrm{GeV}^{2}$ (see discussion in [41]). As a consequence of the Regge behavior of $\Delta q$, the OAM density is peaked at low $x$. Our values for the proton's angular momentum components are: $J_{u}=0.286 \pm 0.011$, $J_{d}=-0.049 \pm 0.007, L_{u}=-0.104 \pm 0.087, L_{d}=0.088 \pm 0.031$ at $Q^{2}=4 \mathrm{GeV}^{2}$.

The total angular momentum density of quarks in the deuteron is compared to the nucleon one in Fig.3.2. The upper panel shows the isoscalar combination, $J_{N}(x)=$ 


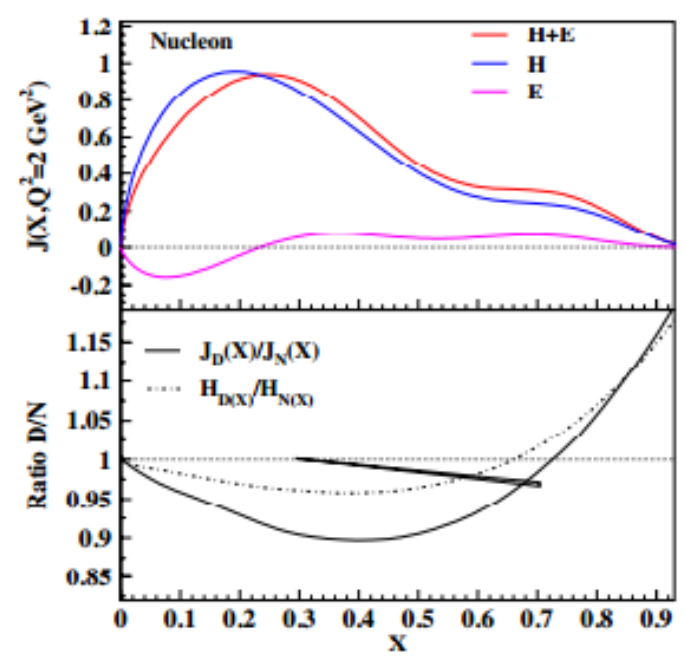

Figure 3.2: (color online) (Upper panel) Contributions $H+E, H$, and $E$, to the integrand in the angular momentum sum rule, Eq.(3.2). All curves were calculated at the scale $Q^{2}=4 \mathrm{GeV}^{2}$, using the parametrization from Ref.[6] for the free nucleon. (Lower panel) The ratio of nuclear to nucleon contributions to angular momentum, $H_{D} / H_{N}$ (dashes), and $H_{2} /\left(H_{N}+E_{N}\right) \equiv J_{D}(x) / J_{N}(x)$, (full curve), calculated using Eq.(3.16) for the deuteron. The small hatched area represents the experimental results from Ref.[40].

$J_{u}(x)+J_{d}(x)$ at $Q^{2}=4 \mathrm{GeV}^{2}$. In the absence of nuclear effects, i.e. if the deuteron were treated as two independently moving nucleons, in Eq.(3.16), $f^{11}(z)=f^{01}(z)=\delta(1-z)$, and $H_{2}=H+E$. Even including nuclear effects, the deuteron angular momentum is dominated by the GPD $H$. The separate dependences of the various components in the deuteron, and their impact on angular momentum are illustrated in the lower panel of Fig.3.2, representing the ratio of the nuclear to nucleon contributions to angular momentum, $H_{D} / H_{N}$ (dashes), and $H_{2} /\left(H_{N}+E_{N}\right) \equiv J_{D}(x) / J_{N}(x)$, (full curve). As in the forward case [42], we find that the distinct angular dependence of the D-wave component plays a non trivial role (more details will be given in [33]) producing a most striking effect through the GPD $E$. Its impact is however suppressed. A similar effect also can be shown for $H_{5}(x, 0,0) \equiv b_{1}$, in agreement with the model calculations of [42].

How does this affect the spin sum rule? On one side, in a deuteron target, we observe that the angular momentum is dominated by the GPD $H$. If the nuclear effects were found to be small, as predicted within a "standard" nuclear model, - nucleons bound by exchanged mesons - the deuteron target would provide an easier access to total angular momentum. On the other side, any deviation from the standard nuclear model predictions presented here would signal a different origin of OAM, perhaps related to gluon components, and would therefore be extremely interesting. The question of whether the quarks' OAM can actually be measured for a deuteron target is therefore mandatory. 
While observables were presented in [43] that contain several deuteron GPDs, none of them is sensitive to $\mathrm{H}_{2}$. Here we suggest the measurement of the deuteron target transverse spin asymmetry, $A_{U T}$, which we derive in terms of GPDs as,

$$
A_{U T} \approx-\frac{4 \sqrt{\tau_{0}}}{\Sigma} \Im m\left[\mathcal{H}_{1}^{*} \mathcal{H}_{5}+\left(\mathcal{H}_{1}^{*}+\frac{1}{6} \mathcal{H}_{5}^{*}\right)\left(\mathcal{H}_{2}-\mathcal{H}_{4}\right)\right]
$$

where $\tau_{0}=\tau(\xi=0), \Sigma$ is the sum of the transversely polarized target cross sections, and $\mathcal{H}_{i}$, are the Compton form factors for the corresponding GPDs. One can see that the term containing $\mathcal{H}_{2}$ should dominate the asymmetry, given the expected smallness of $\mathcal{H}_{5}[13,14]$.

In conclusion, we analyzed the question of OAM in a spin one hadronic system. We derived a sum rule whereby the second moment of the GPD $H_{2}$ gives the total angular momentum, $\mathrm{H}_{2}$ being the same GPD whose first moment gives the magnetic moment. Nuclear effects evaluated within a standard model for the deuteron give $H_{2} \approx H+E$, that is the quarks' angular momenta in the deuteron, and hence their OAM, are predicted to be similar to the sum of the neutron plus proton taken alone. This cancellation is consistent with the smallness of the deuteron magnetic moment, reflecting the approximate cancellation between the proton and neutron magnetic moments. If found in experiment, deviations from this standard behavior which is calculable to high precision and under control, could be a signal of other degrees of freedom such as six quark components, or $k_{\perp}$ dependent re-interactions beyond the collinear convolution considered here. In either situation studying spin one hadronic systems might shed light on the elusive gluon angular momentum components. Finally, we show that measuring angular momentum in the deuteron can be at reach in future experimental facilities with high enough energy and luminosity, through transverse spin observables.

\subsection{Helicity Amplitudes: Formalism}

We now explore the helicity amplitudes for DVCS from a spin-1 hadron, for the purpose of identifying observables that would give access to quark orbital angular momentum for spin-1 hadrons. We know that $\frac{1}{2} \int d x H_{2}(x, 0,0) x=1$ and we will try to explore the various possiblities of accessing the magnetic form factor GPD, $H_{2}$, of the deuteron. The observables in consideration are the amplitudes for photon-deuteron scattering as used in DVCS, given by $f_{\Lambda_{\gamma} \Lambda, \Lambda_{\gamma}, \Lambda^{\prime}}$; where $\Lambda_{\gamma}$ denotes the helicity of the initial (virtual) photon, $\Lambda$ denotes the initial helicity of the deuteron, $\Lambda_{\gamma^{\prime}}$ denotes the helicity of the outgoing photon and $\Lambda^{\prime}$ the final helicity of the deuteron. The momentum four-vectors 
for the photon, initial state of the deuteron and the final state of the deuteron are, respectively, $q^{u}, p^{u}$ and $p^{u^{\prime}}$ and are not chosen in any particular frame for the moment. The quark-current correlation function, which contains all the information on the soft contribution to the unpolarized helicity amplitudes, reads [14],

$$
\begin{aligned}
& \int \frac{d \kappa}{2 \pi} e^{i x P^{+} \kappa}<p^{\prime}, \lambda^{\prime}|\bar{\psi}(-k n) \gamma \cdot n \psi(k n)| p^{\prime}, \lambda^{\prime}> \\
& =\epsilon^{\prime *} \cdot \epsilon H_{1}+\frac{\epsilon^{\prime *} \cdot P \epsilon \cdot n+\epsilon \cdot P \epsilon^{\prime *} \cdot n}{P \cdot n} H_{2} \\
& -\epsilon^{\prime *} \cdot P \epsilon_{1} \cdot P \frac{H_{3}}{2 M^{2}}+\frac{\epsilon^{\prime *} \cdot P \epsilon \cdot n-\epsilon \cdot P \epsilon^{\prime *} \cdot n}{P . n} H_{4} \\
& +\left(\frac{4 M^{2} \epsilon^{\prime *} \cdot n \epsilon \cdot n}{(P . n)^{2}}+\epsilon^{\prime *} \cdot \epsilon \frac{1}{3}\right) H_{5}
\end{aligned}
$$

This correlator, which contains the unpolarized GPDs, contributes to the symmetric (in Lorentz indices) soft part of the helicity amplitude. Another correlation function appears, related to the axial-vector current. We however disregard it in what follows since it does not contribute to the angular momentum sum rule.

The quark-current correlator can be written in the form

$$
\epsilon^{\mu}(p, \Lambda) \epsilon^{\prime * \nu}\left(\Lambda^{\prime}, p^{\prime}\right) L_{\mu \nu}\left(p^{\prime}, p\right)
$$

where $\epsilon_{\mu(\nu)}$ is the polarization Lorentz four-vector of the spin-1 deuteron and $L_{\mu \nu}$ is a rank-2 tensor, explicitly

$$
\begin{aligned}
L_{u v} & =g_{u v} H_{1}(x)+\frac{n_{u} P_{v}+n_{v} P_{u}}{P^{+}} H_{2}(x) \\
& -\frac{P_{u} P_{v}}{2 m^{2}} H_{3}(x)+\frac{n_{u} P_{v}-n_{u} P_{v}}{P^{+}} H_{4}(x) \\
& +\left[\frac{4 m^{2} n_{u} n_{v}}{P^{+2}}+\frac{g_{u v}}{3}\right] H_{5}(x)
\end{aligned}
$$

Moreover, each term in the quark-current can be written in the form

$$
\epsilon^{\mu}(p) \epsilon^{\prime * \nu}\left(p^{\prime}\right) L_{\mu \nu}^{i}\left(p^{\prime}, p\right)
$$


and $L_{u v}$ above can of course be rewritten in terms of the particular $L_{u v}^{i}$ 's $(\mathrm{i}=1, \ldots, 5)$ as

$L_{u v}=\Sigma L_{u v}^{i}$, where

$$
\begin{aligned}
L_{u v}^{1} & =g_{u v} H_{1}(x) \\
L_{u v}^{2} & =\frac{n_{u} P_{v}+n_{v} P_{u}}{P^{+}} H_{2}(x) \\
L_{u v}^{3} & =-\frac{P_{u} P_{v}}{2 m^{2}} H_{3}(x) \\
L_{u v}^{4} & =\frac{n_{u} P_{v}-n_{u} P_{v}}{P^{+}} H_{4}(x) \\
L_{u v}^{5} & =\left[\frac{4 m^{2} n_{u} n_{v}}{P^{+2}}+\frac{g_{u v}}{3}\right] H_{5}(x)
\end{aligned}
$$

The Lorentz structure comes from the particle's four-momenta and general longitudinal light-cone four-vectors like $n^{u^{\prime}}=(1,0,0,-1)$ [14].

Now, we recast the current as

$$
\begin{aligned}
& V_{\Lambda^{\prime} \Lambda} \\
= & \mathbf{K}_{\mu \nu \nu}\left(\mathbf{p}^{\prime}, \mathbf{p}\right) \epsilon^{\mu \prime}(\mathbf{0}) \epsilon^{\prime * \nu^{\prime}}(\mathbf{0}) \\
= & \Sigma K_{\mu^{\prime} \nu^{\prime}}^{i}\left(p^{\prime}, p\right) \epsilon^{\mu^{\prime}}(0, \Lambda) \epsilon^{\prime * \nu^{\prime}}(0, \Lambda),
\end{aligned}
$$

where $\mathbf{K}_{\mu^{\prime} \nu^{\prime}}\left(\mathbf{p}^{\prime}, \mathbf{p}\right) \equiv \mathbf{S}_{\mu^{\prime} \nu^{\prime}}^{\mu \nu}\left(\mathbf{p}^{\prime}, \mathbf{p}\right) \mathbf{L}_{\mu \nu}\left(\mathbf{p}^{\prime}, \mathbf{p}\right)$ is a rank-2 tensor that is contracted with the rest frame polarization vectors of the spin-1 target. Term by term, $K_{\mu^{\prime} \nu^{\prime}}^{i}\left(p^{\prime}, p\right) \equiv$ $S_{\mu^{\prime} \nu^{\prime}}^{\mu \nu}\left(p^{\prime}, p\right) L_{\mu \nu}^{i}\left(p^{\prime}, p\right)$. (Here, $x=\frac{k^{+}}{P^{+}}$and $\left.\Delta=p^{\prime}-p\right)$. $S_{\mu^{\prime} \nu^{\prime}}^{\mu \nu}$ is a rank-4 tensor that Lorentz-transforms the product $\epsilon_{\mu}(0) \epsilon_{\nu}(0)$ to their desired momenta, using a combination of appropriate boosts and rotations. It can be written in this way: $S_{\mu \nu}^{\mu^{\prime} \nu^{\prime}}=T_{\mu^{\prime}}^{\mu} T_{\nu^{\prime}}^{\nu}$, where the $T$ matrices are Lorentz transformation matrices. It is explicitly processdependent. Thus, it is a direct tensor product of two Lorentz transformations, and is the cost of writing the polarization vectors in their rest frame. So, the quark-current can be rewritten in terms of just the rest frame polarization vectors and a rank-two tensor. The primed Lorentz index is used here for polarization vectors in the rest frame and the primed field index is used to indicate final state. As shown above, this decomposition is also valid for each term in the current. For calculating helicity amplitudes, these restframe polarization four-vectors are chosen to have spin projection $(+1,0$ or- 1$)$ along the 
$z$-axis, and then they are constructed to their desired general form using the tensor $S_{\mu^{\prime} \nu^{\prime}}^{\mu \nu}$

Aside: We have used here the fact that the form of the polarization vector only depends on the spin and momentum of the hadron in any frame, and thus we can construct it by boosting from rest to the appropriate frame, and use the result in any frame. For example, we may have two different hadrons with different momenta in the same frame, but their boosted-from-rest free-field form can be used here for both the hadrons.

For example, here is $K_{\mu^{\prime} \nu^{\prime}}^{1}$ for general longitudinal scattering (initial and final deuteron momenta $p^{\mu}=\left(E, 0, p_{3}\right), p^{\prime \mu}=\left(E^{\prime}, 0, p_{3}^{\prime}\right)$ respectively $)$ :

$$
K_{\mu^{\prime} \nu^{\prime}}^{1}=\frac{H 1}{m^{2}}\left[\begin{array}{cccc}
-p^{3} p^{\prime 3}+E E^{\prime} & 0 & 0 & E p_{3}^{\prime}-E^{\prime} p_{3} \\
0 & -1 & 0 & 0 \\
0 & 0 & -1 & 0 \\
E^{\prime} p_{3}-E p_{3}^{\prime} & 0 & 0 & p^{3} p^{\prime 3}-E E^{\prime}
\end{array}\right]
$$

Of course, we can incorporate photons into $L_{\mu \nu}$ as well and preserve this structure of the helicity amplitude.

Let us go one step further and construct a general formula for the soft helicity amplitudes as a function of the helicity of the deuteron, $\Lambda=-1,0$ or 1 . We realize that the polarization vector $\epsilon^{\mu}$ (which equals $(0,1, i, 0),(0,-1, i, 0),(0,0,0,1)$ for spins $+1,-1,0$ respectively) can be written generally in the rest frame as:

$\epsilon^{\mu}(0, \Lambda)=(0, \Lambda, i|\Lambda|, 1-|\Lambda|)$

Thus, $V_{\Lambda^{\prime} \Lambda}=K_{\mu^{\prime} \nu^{\prime}} \epsilon^{\mu^{\prime}}(0, \Lambda) \epsilon^{\prime * \nu^{\prime}}\left(0, \Lambda^{\prime}\right)$.

Thus, we have stripped the momentum dependence from the helicity dependence in the helicity amplitudes. Now, we have a general form for the polarization vectors in any frame. Denoting $p^{\mu}=\left(E, p_{T}, p_{3}\right)\left(|p|\right.$ is the magnitude of the three-momentum, $p_{3}$ is the momentum along $z, p_{T}$ is the transverse momentum component to the z-axis and the deuteron is in a helicity eigenstate), 


$$
\epsilon^{\mu}\left(\vec{p}=\left(p_{T}, p 3\right), \Lambda=s 1\right)=\left(\frac{|p|(1-|s 1|)}{\sqrt{2} m}, \frac{p_{T} p_{3} s 1}{\sqrt{2} p_{T}|p|}+\frac{p_{T} E(1-|s 1|)}{\sqrt{2} m|p|}, \frac{i p_{T}|s 1|}{\sqrt{2} p_{T}},-\frac{p_{T} s 1}{\sqrt{2}|p|}+p_{3} E \frac{(1-|s 1|)}{\sqrt{2} m|p|}\right)
$$

In particular, for a deuteron moving along the $z$-axis with momentum $p_{3}$, we obtain:

$$
\epsilon^{\mu}\left(\vec{p}=\left(0, p_{3}\right), \Lambda=s 1\right)=\left(\frac{p_{3}(1-|s 1|)}{\sqrt{2} m}, \frac{s 1}{\sqrt{2}}, \frac{i|s 1|}{\sqrt{2}}, E \frac{(1-|s 1|)}{\sqrt{2} m}\right)
$$

Another advantage of this formulation, besides a common formulation for all hadronic helicity amplitudes and neatness of expression, is that we have separated the momentum and spin-dependent parts of the helicity amplitude. We only need ask what is happening to the rest-frame spin projection to account for changes in the overall helicity amplitude. The rest-frame spin direction is never mixed with perpendicular boosts and is cleanly retained in the construction. We obtain the general formula for all 9 helicity amplitudes (the reason for the number 9 of twist-two spin-one GPDs). The coefficient of $H_{1}$ in the general longitudinal scattering case (initial deuteron momentum $p^{3}$ and final $p^{\prime 3}$ ) only is given below for reasons of compactness:

$$
\begin{aligned}
& \frac{p^{\prime 3} s 1 s 2}{\left.2 p^{\prime 3}-\frac{p 3 p^{\prime 3}}{(} 1-A b s(s 1)\right)(1-A b s(s 2))} 2 m^{2} \\
+ & \frac{\sqrt{m^{2}+p 3^{2}} p^{\prime 3} \sqrt{m^{2}+p f 3^{2}}(1-A b s(s 1))(1-A b s(s 2))}{2 m^{2} p^{\prime 3}}-\frac{1}{2} A b s(s 1) A b s(s 2)
\end{aligned}
$$

\subsubsection{Simple Examples of Transformations}

So, what is the structure of $S_{\mu^{\prime} \nu^{\prime}}^{\mu \nu}$ ? It depends, of course, on the initial and final momenta of our spin-1 target, which decide precisely what observable we are seeking. Our goal is to identify an observable which gives us relatively easy access to the GPD $H_{2}$. It is important to note here that momentum and spin do not transform in the same way: four-momentum of course transforms like a four-vector under Lorentz Transformations, but spin does not. We exploit the fact that spin is unchanged when one boosts along the axis of spin-projection and produce states that are eigenstates of helicity. First, let us look at these amplitudes in a Breit frame, just as an example, in which the momenta lie along and opposite to $\hat{z}$. Here 


$$
S_{\mu^{\prime} \nu^{\prime}}^{\mu \nu}=\Lambda_{\mu}^{\mu^{\prime}}(-|p| \hat{z}) \Lambda_{\nu}^{\nu^{\prime}}(+|p| \hat{z})
$$

where $\Lambda_{\mu}^{\mu^{\prime}}$ is the Lorentz Boost Matrix and $|p|$ is the magnitude of the three-momentum (z-momentum here) of the hadron. These are given in Table 1.

All the helicity amplitudes are of course Lorentz Invariant - meaning that the same process when viewed from a different Lorentz frame, boosted or rotated, though not a helicity amplitude as boosts and rotations do not commute, will be the same probability amplitude as it is just the same process from a different "vantage point" - and hence rotating to a frame where the helicity states point in an arbitrary direction will not change the amplitudes. However, it is instructive to start working in a generalized Breit Frame nevertheless and then proceed to a frame with final transverse momentum components and compare the two results. One Lorentz-transformation matrix will act on the initial polarization vector (in a state of definite spin projection in $z$ : we will choose $\Lambda=+1$ in the rest frame), rotate it to polar angles $\phi$ and $\theta$ and then give it momentum in along this direction by means of a boost. The other matrix will rotate and boost the final polarization vector (again, chosen to be the same spin for simplicity) to a direction opposite the above rotated vector. The first matrix will be a left-to-right product of the boost matrix taken along the $z$-axis, i.e. $\Lambda_{u}^{u^{\prime}}(-|p| \hat{z}), R_{y}(\theta)$ and $R_{z}(\phi)$, where $R_{i}$ denotes the 4-dimensional Lorentz matrix that rotates counterclockwise about axis $i$ by the specified angle. Here, the initial deuteron momentum is given by $p^{\mu}=\left(E, p_{1}, p_{2}, p_{3}\right)$ and the final by $p^{\prime \mu}=\left(E^{\prime}, p_{1}^{\prime}, p_{2}^{\prime}, p_{3}^{\prime}\right)$.

The matrix is:

$$
T_{\mu^{\prime}}^{\mu}=R_{z}(\phi) R_{y}(\theta) \Lambda_{\mu^{\prime}}^{\mu}(-|p| \hat{z})
$$

where $|p|=\sqrt{\left(p_{1}\right)^{2}+\left(p_{2}\right)^{2}+\left(p_{3}\right)^{2}}$ is the magnitude of the 3-momentum of the hadron and the indices on the right-hand-side have loosely been assigned to the boost matrix alone. In matrix form,

$$
T_{\mu^{\prime}}^{\mu}=\left[\begin{array}{cccc}
1 & 0 & 0 & 0 \\
0 & \cos (t) \cos (\phi) & -\sin (\phi) & \cos (\phi) \sin (t) \\
0 & \sin (\phi) \cos (t) & \cos (\phi) & \sin (\phi) \sin (t) \\
0 & -\sin (t) & 0 & \cos (t)
\end{array}\right]\left[\begin{array}{cccc}
E / m & 0 & 0 & |p| / m \\
0 & 1 & 0 & 0 \\
0 & 1 & 0 & 0 \\
|p| / m & 0 & 0 & E / m
\end{array}\right]
$$


where $\cos (t)=\frac{p_{3}}{|p|}, \tan (\phi)=\frac{p_{2}}{p_{1}}, m$ is the mass of the deuteron and $E=\sqrt{m^{2}+|p|^{2}}$ its energy. This gives

$$
T_{\mu^{\prime}}^{\mu}=\left[\begin{array}{cccc}
\frac{E}{m} & 0 & 0 & \frac{|p|}{m} \\
\frac{p_{1}}{m} & \frac{p_{1} p_{3}}{p_{T}|p|} & -\frac{p_{2}}{p_{T}} & \frac{E p_{1}}{m|p|} \\
\frac{p_{2}}{m} & \frac{p_{2} p_{3}}{p_{T}|p|} & \frac{p_{1}}{p_{T}} & \frac{E p_{2}}{m|p|} \\
\frac{p_{3}}{m} & -\frac{p_{T}}{|p|} & 0 & \frac{E p_{3}}{m|p|}
\end{array}\right],
$$

where $p_{T}=\sqrt{\left(p_{1}\right)^{2}+\left(p_{2}\right)^{2}}$ and $b=\sqrt{1-\left(\frac{p_{3}}{|p|}\right)^{2}}$. Since the rotation is about the $y$ and $z$ axes, the matrix components look different in terms of the 3 directions. They would not treat all vectors the same due to the order of operations we chose. It is true, however, that this matrix rotates a rest-frame momentum four-vector to point in the direction given by the final momentum vector $\vec{p}=\left(p_{1}, p_{2}, p_{3}\right)$ without any bias to a particular direction. This is evident by its first column only. That is,

$T_{\mu^{\prime}}^{\mu}(m, 0,0,0)=\left(E, p_{1}, p_{2}, p_{3}\right)$

(transpose of row-vectors again implied in notation). Note that to obtain a polarization vector with helicity in exactly the opposite direction, one does not simply multiply by a negative $3 \mathrm{x} 3$ identity matrix as for ordinary vectors like momentum, which has components only in the plane of rotation. One rotates the boosted positive helicity $(z)$ polarization vector by $\pi$ about the $x$-axis (thus negating its $y, z$ components), and then proceed with the same rotations as for the above case. Basically, one is getting a state with momentum and spin that point opposite to that $\left|+p_{3} s_{z}=+1\right\rangle$ helicity eigenstate and then rotating the frame like usual to end up with it still pointing opposite to the rotated positive helicity vector. So, we get two states pointing opposite to each other in momentum and spin.

Aside: One can boost parallel to the spin direction before or after a rotation - the vector will clearly be the same. It is seen above that boosts longitudinal to the spin direction and any rotation commute, the trick being that the boost axis, though always parallel to the spin direction, is different when the rotation is done before the boost vs after.

$$
T_{\nu^{\prime}}^{\nu}=R_{z}(\phi) R_{y}(\theta) R_{x}(\pi) \Lambda_{\nu^{\prime}}^{\nu}(-|p| \hat{z})
$$




$$
T_{\nu^{\prime}}^{\nu}=\left[\begin{array}{cccc}
\frac{E}{m} & 0 & 0 & \frac{|p|}{m} \\
-\frac{p_{1}}{m} & \frac{p_{1} p_{3}}{p_{T}|p|} & \frac{p_{2}}{p_{T}} & -\frac{E p_{1}}{m|p|} \\
-\frac{p_{2}}{m} & \frac{p_{2} p_{3}}{p_{T}|p|} & -\frac{p_{1}}{p_{T}} & -\frac{E p_{2}}{m|p|} \\
-\frac{p_{3}}{m} & -\frac{p_{T}}{|p|} & 0 & -\frac{E p_{3}}{m|p|}
\end{array}\right],
$$

Then we have $S_{\mu \nu}^{\mu^{\prime} \nu^{\prime}}=T_{\mu^{\prime}}^{\mu} T_{\nu^{\prime}}^{\nu}$ for this frame.

It is clear that $p_{z}$ appears differently in the transformation because of the choice of initial polarization vector. Incidentally, if there is a phase from a rotation that we have not accounted for, it will only be an overall phase since each term has the same objects that are to be transformed identically.

One now sees that this decomposition is in perfect analogy with the spin $\frac{1}{2}$ case. As in [6], we see that terms in the soft part of these amplitudes for the proton take the form

$$
\bar{u}\left(p^{\prime}, \Lambda^{\prime}\right)\left[p^{\prime}, p\right] u(p, \Lambda)
$$

where $u(p)$ is the usual four-component Dirac spinor and $\left[p^{\prime}, p\right]$ contains dotted Dirac gamma matrices and scalars. This expression can be conveniently written in terms of rest-frame spinors as $\bar{u}(0)\left[p^{\prime}, p\right] u(0)$, where $\left[p^{\prime}, p\right]$ now denotes a revised matrix which incorporates the effect of boosting the spinors from their rest frame and is a function of the initial and final target four-momentum. Our $K_{\mu / \nu /}\left(p^{\prime}, p\right)$ is precisely that.

Since $V_{\lambda^{\prime} \lambda}$ are the unpolarized GPDs, they do not necessarily have to enter amplitudes for polarized scattering. In fact, as we will shortly see, most of them do not enter scattering in a longitudinal frame. From the above relations, we can solve for $\mathrm{H}_{2}$ in terms of the other GPDs:

$H_{2}=\frac{m^{2}}{2|p|^{2}}\left[\mathbf{V}_{\mathbf{0 0}}-H_{1}\left(-1-2 \frac{|p|^{2}}{m^{2}}\right)+2 \frac{|p|^{2}}{m^{2}} H_{3}\left(1+\frac{|p|^{2}}{m^{2}}\right)-2 H_{4}|p| \frac{\sqrt{m^{2}+|p|^{2}}}{m^{2}}+\frac{2}{3} H_{5}\left(1-2 \frac{|p|^{2}}{m^{2}+|p|^{2}}\right)\right]$ 
where $V_{00}$ is the observable quantity (after integration with respect to $x$ ) and the other GPDs are accessible via their Compton Form Factors from other processes [13].

It is important to note that if the momenta are large, then the amplitude would tend to diverge unless the Compton Form Factors suppressed the result by at least $\frac{1}{\Delta^{2}}$ (in this frame it is convenient to see since $\Delta^{2}=|p|^{2}$ ). Now, the expression above is of course Lorentz Invariant, but not manifestly so. Boosting to a more familiar frame (large boost along $-\hat{z}$ ), and with $p$ and $p^{\prime}$ now temporarily referring to the hadron momenta in this frame, one finds

$$
\epsilon^{\prime *} \cdot \epsilon=\frac{p_{3} \cdot p_{3}^{\prime}-E \cdot E^{\prime}}{m^{2}}=-1-2 \frac{|p|^{2}}{m^{2}}+H . O .
$$

Writing the above in terms of more familiar variables,

$$
\epsilon^{\prime *} . \epsilon=\frac{-p^{+}}{p^{\prime+}}=-\frac{1+\xi}{1-\xi}
$$

One finds that $\xi \rightarrow 1$ in any frame that is boostable to a Breit Frame with large momenta along the 3 -axis (not all off-forward processes are boostable to a large-momenta Breit frame). Thus in all such infinite-momentum frames, one sees that the CFFs must be at least linear in $1-\xi$. The different coefficients in $V_{00}$, in longitudinal off-forward scattering, are given as follows:

$$
\begin{aligned}
\left(H_{1}\right) \epsilon^{\prime *} . \epsilon & -\frac{1+\xi}{1-\xi}\left(H_{1}\right) \\
\left(H_{2}\right) \frac{\epsilon^{\prime *} . P \epsilon \cdot n+\epsilon \cdot P \epsilon^{\prime *} . n}{P . n} & \frac{2 \xi}{1-\xi}\left(H_{2}\right) \\
\left(H_{3}\right) \epsilon^{\prime *} \cdot P \epsilon_{1} \cdot P \frac{1}{2 M^{2}} & {\left[\frac{2 \xi}{1-\xi}+2 \frac{\xi^{2}}{1-\xi^{2}}\right]\left(H_{3}\right) } \\
\left(H_{4}\right) \frac{\epsilon^{\prime *} \cdot P \epsilon \cdot n-\epsilon \cdot P \epsilon^{\prime *} \cdot n}{P . n} & \frac{2 \xi}{1-\xi}\left(H_{4}\right) \\
\left(H_{5}\right)\left(\frac{4 M^{2} \epsilon^{\prime *} \cdot n \epsilon \cdot n}{(P . n)^{2}}+\epsilon^{\prime *} \cdot \epsilon \frac{1}{3}\right) & \frac{2}{3}\left(H_{5}\right)
\end{aligned}
$$




\begin{tabular}{c|c|c|c}
\hline Amp & Fwd & Longitudinal & General \\
\hline$V_{++}$ & $H_{1}-\frac{H_{5}}{3}$ & $H_{1}-\frac{H_{5}}{3}$ & $\frac{H_{1}}{2}\left(1+\frac{p^{\prime 3}}{\left|\vec{p}^{\prime}\right|}\right)+\frac{H_{2} p_{T}^{2}}{2\left|\vec{p}^{\prime}\right|\left(p^{+}+p^{\prime+}\right)}+\frac{H_{3} p_{T}^{2} p^{3}}{4 m^{2}\left|\vec{p}^{\prime}\right|}-\frac{H_{4} p_{T}^{2}}{\left.2\left|\vec{p}^{\prime}\right| \mid p^{+}+p^{\prime+}\right)}-\frac{H_{5}}{6}\left(1+\frac{p^{\prime 3}}{p^{0}}\right)$ \\
$V_{0+}$ & 0 & 0 & *insufficient space* \\
$V_{+0}$ & 0 & 0 & *insufficient space* \\
$V_{00}$ & $H_{1}+\frac{2 H_{5}}{3}$ & & *insufficient space* \\
$V_{+-}$ & 0 & 0 & $\frac{H_{1}}{2}\left(1-\frac{p^{\prime 3}}{\left|\vec{p}^{\prime}\right|}\right)-\frac{H_{2} p_{T}^{2}}{2\left|\vec{p}^{\prime}\right|\left(p^{+}+p^{\prime+}\right)}+\frac{H_{3} p_{T}^{2} p^{3}}{4 m^{2}\left|\vec{p}^{\prime}\right|}+\frac{H_{4} p_{T}^{2}}{2\left|\vec{p}^{\prime}\right|\left(p^{+}+p^{\prime+}\right)}+\frac{H_{5}}{6}\left(\frac{p^{\prime 3}}{p^{\prime 0}}-1\right)$ \\
\hline
\end{tabular}

TABLE 3.1: Unpolarized Helicity Amplitudes (Soft Part)

\begin{tabular}{c|c}
\hline Amp & Transverse \\
\hline$V_{++}$ & $\frac{H_{1}}{2}+\frac{H_{2} p_{T}}{2\left(p^{+}+p^{\prime+}\right)}+\frac{H_{3} p_{3} p_{T}}{4 m^{2}}-\frac{H_{4} p_{T}}{2\left(p^{+}+p^{\prime+}\right)}-\frac{H_{5}}{6}$ \\
$V_{+0}$ & $\frac{H_{1} p_{T} p_{0}^{\prime}}{\sqrt{2} m p_{T}^{\prime}}-\frac{H_{2} p_{T}^{2}}{\sqrt{2} m\left(p^{+}+p^{\prime+}\right)}+\frac{H_{3} p^{0} p_{T}^{2}}{2 \sqrt{2} m^{3}}+\frac{H_{4} p_{T}^{2}}{\sqrt{2} m\left(p^{+}+p^{\prime+}\right)}-\frac{H_{5} p^{0}}{\sqrt{2} m}$ \\
$V_{+-}$ & $\frac{H_{1}}{2}-\frac{H_{2} p_{T}}{2\left(p^{+}+p^{\prime+}\right)}-\frac{H_{3} p_{3} p_{T}}{4 m^{2}}+\frac{H_{4} p_{T}}{2\left(p^{+}+p^{\prime+}\right)}-\frac{H_{5}}{6}$ \\
$V_{0+}$ & $-\frac{H_{1} p^{\prime}}{\sqrt{2} m}+\frac{H_{2} p_{3}}{\sqrt{2} m}\left(p^{+}-\frac{p^{\prime+}}{p^{+}+p^{\prime+}}\right)-\frac{H_{3} p^{2} p^{0}}{2 \sqrt{2} m^{3}}+\frac{H_{4} p_{3}}{\sqrt{2} m}\left(p^{+}+\frac{p^{\prime+}}{p^{+}+p^{\prime+}}\right)+\frac{H_{5}}{\sqrt{2} m}\left(\frac{p^{0}}{3}-\frac{4 m^{2} p^{+}}{\left(p^{+}+p^{\prime+}\right)^{2}}\right)$ \\
$V_{00}$ & *Insufficient Space* \\
\hline
\end{tabular}

TABle 3.2: Transverse Unpolarized Helicity Amplitudes (Soft Part)

In Table 1, we see all the five independent unpolarized amplitudes. The second column lists amplitudes for forward longitudinal scattering, the third column lists amplitudes for general longitudinal scattering in terms of momenta $p^{3}$ and $p^{\prime 3}$ and the fourth column gives amplitudes for the case that the final state also has a transverse component, $p_{T}$. Table 2 lists the special case of purely transverse scattering, i.e. $\xi=0$. It is easily checked that the amplitudes above reduce to their tabular counterparts in the appropriate limits (in particular to eqns 97-98 of [14] in the forward limit). We can correctly solve for $\mathrm{H}_{2}$ using any combination of the independent helicity amplitudes listed.

In the forward limit, it is easy to check that they reduce to Cano-Pire eqns 97-98 in the appendix of their "Deep Electroproduction" paper (2003). It is interesting that $V_{+0}$ is the only amplitude that has no direct transverse-momentum dependence ( $V_{00}$ does). So, the fact that the final spin projection does not have a particular direction (0) added with the fact that the initial spin projection does have one, gives us a form for the amplitude that does not directly depend on the final transverse momentum. Also, the forms are not as complicated if the final helicity is 0 , but do get complicated somehow if the initial helicity is zero. 


\subsubsection{Accessing the Compton Form Factors}

Thus, we have outlined all the soft scattering amplitudes. If one wishes to have access to a particular Compton Form Factor $(\mathrm{CFF}), \int d x \frac{H_{i}\left(x, t=-\Delta^{2}, \zeta\right)}{x-\zeta+i \epsilon}$, one just needs to reversesolve the above in terms of the helicity amplitudes, as a function of $t, \zeta$ and the observed amplitude itself, $V_{\Lambda^{\prime} \Lambda}$. Note that if one just used the longitudinal scattering amplitudes (in any frame, Breit or other), one does not get 5 linearly independent equations to solve, as most of the amplitudes are zero. Also, all longitudinal helicity amplitudes except $V_{00}$ are independent of kinematical variables. However, the CFFs can be accessed at the same kinematical value as originally desired by using the 3 effective degrees of freedom $\left(p^{3}, p^{\prime 3}, p_{T}\right)$ to arrive at the $t, \zeta$ of our choosing. In fact, even 2 degrees of freedom suffice, so the purely transverse scattering amplitudes can also be used. Then, the CFFs can be written as a general function of the desired $t, \zeta$ after solving the above 5 amplitudes for them and the experiment can be designed according to the specific target momenta these two variables translate into.

Since $t$ and $\zeta=\Delta^{+} p^{+}$remain the same under the above transformation of variables from the case of longitudinal scattering to purely transverse scattering, one can imagine this to be a Lorentz transformation from the original frame. However, the coefficients to the CFFs in the soft amplitude do change with the choice of frame, as the spins are rotated to give us helicity states in all frames. It is not a real Lorentz transformation, though, as $\zeta$ does not change despite a transverse component to the boost yielding the purely transverse frame. The formalism for GPDs guarantees that the observed helicity amplitudes will be forced to change in a way that is in agreement with the GPDs remaining fixed in value.

These polarization vectors have information about the spin, just like the Dirac spinor does for the proton. At a certain momentum (resulting from a particular tranformation of the spinor from the rest frame with fixed spin) one has a certain form for the spinor, with a certain expectation value of spin. It is generally not an eigenstate of spin or helicity unless one boosts along the direction of initial spin projection. The situation is the same for the polarization vectors, where their rest-frame form unambiguously tells us the spin direction. For example $\epsilon^{+-}(0,1,+-i, 0)$ tell us the spin direction that map to the polarizations of classical optics. One should note that the helicity amplitudes are "frame-invariant" only in the IMF, and thus the invariant form can't really be advertised as such. 


\section{Chapter 4}

\section{Simple Physical Arguments Related to Angular Momentum}

In this chapter, we shall go over some simple physically pertinent observations related to hadronic angular momentum, and some small relevant calculations. A lot of the observations stand alone, though they are all connected to each other in spirit. They will involve looking at spin and orbital angular momentum in different reference frames, in position and momentum space, and will use various symmetry and homogeneity arguments.

\subsection{Boost-like Operators}

\subsubsection{Commutation of Boost and Rotations}

Boosts and rotations referring to the same axis commute for Dirac spinors. This is seen as follows:

1) The state $\left|+_{R}\right\rangle$, the spin-up state in the $\hat{z}$ basis, and $\left|{ }_{R}\right\rangle$, the spin-down state in the rest frame, are both eigenstates of $J^{z}$. This is seen by azimuthal symmetry under a rotation about the $z$ axis effected on these states by the rotation operator $J^{z}$, which returns to us the same state with a phase of rotation. The spin projection is also unaffected by a parallel boost (along $z$-axis) by azimuthal symmetry. There is no preferred transverse direction available. 
Thus, the eigenvalue of $J^{z}$ acting on the boosted state is the same as that in the rest frame, again by azimuthal symmetry in the boosted frame (and by spin quantization). This means that the two operators commute.

The above arguments apply directly to all eigenstates of $J^{z}$ for any system- boosts and rotations commute for a complete basis (the $\hat{z}$ basis). This means that the boost and rotation operators referencing the same axis generally commute! Thus, this is true not just for 4-vectors as is generally known but for spinors as well.

2) Due to quantization, the value of the spin of a proton cannot change either along that axis. If the spin of the system is higher, even then it will not change and this will be shown shortly.

3) In other words, a state, $|\chi\rangle$, when boosted by the spin $\frac{1}{2}$ Lorentz Transformation matrix, does not change its expectation value of spin in the direction of boost:

$\left\langle\chi\left|\Lambda_{\frac{1}{2}}^{-1} J^{z}\right| \Lambda_{\frac{1}{2}} \chi\right\rangle=\left\langle\chi\left|\Lambda_{\frac{1}{2}}^{-1}\right| \Lambda_{\frac{1}{2}} J^{z} \chi\right\rangle=\left\langle\chi\left|J^{z}\right| \chi\right\rangle$

Thus, the same angular momentum of a state is unchanged along the direction of boost.

4) This means that $\left\langle x+\left|J^{z}\right| x+\right\rangle=0$ before and after boosting. ( $|x+\rangle$ is referring to the boosted- to- positive- $z$-momentum transverse-spin state, projecting in the $x$ direction in the rest frame). It is zero before the boost again by azimuthal symmetry.

This fact that can be seen another way:

$$
\begin{aligned}
\left\langle x+\left|J^{z}\right| x+\right\rangle & =\left\langle+\left|J^{z}\right|+\right\rangle+\left\langle-\left|J^{z}\right|-\right\rangle \\
& +\left\langle+\left|J^{z}\right|-\right\rangle+\left\langle-\left|J^{z}\right|+\right\rangle \\
& =0
\end{aligned}
$$

We have used the linearity of boosts above by rewriting the $|x+\rangle$ state as a boost acting on the rest-frame transverse-spin state, and rewritten the latter in terms of $z$-spin 
eigenfunctions. This was then boosted. It is clear that the first two terms on the RHS above cancel and the last two terms are zero by orthogonality.

5) All of the above is after all intuitive: angular momentum should not change in the direction of boost since it is described by quantities behaving like $\vec{r} \times \vec{p}$, and one cannot add a component parallel to the direction of change of momentum. This holds true in the quantum theory as well, even though transverse projection distribution would change and does depend on the longitudinal state.

6) However, all bets are off when speaking of boosts transverse to the direction of measurement. In the rest frame, $\left\langle x+\left|J^{x}\right| x+\right\rangle=\frac{1}{2}$. However, it is intuitively obvious that boosting in a direction transverse to spin projection will mix spin directions. In any Lorentz Transformation, fields and coordinates have transformation laws, and there is no obvious symmetry here that dictates that transverse spin projection will remain the same. One may argue that the above matrix element can be rewritten, after using azimuthal symetry again, as:

$\left\langle x+\left|J^{x}\right| x+\right\rangle=\left\langle+\left|J^{x}\right|-\right\rangle+\left\langle-\left|J^{x}\right|+\right\rangle$,

and thus by symmetry about the $z$ axis the two matrix elements above should disappear. However, they are off-forward elements, not a physical observable by themselves, and can involve phases that give physical values. After all, we have made a choice of basis where there is arbitrariness of phase relative to the right-handed coordinate system etc. Indeed, in the rest frame, these two terms, $\left\langle x_{R}+\left|J^{x}\right| x_{R}+\right\rangle+\left\langle x_{R}+\left|J^{x}\right| x_{R}+\right\rangle$, add to give $\frac{1}{2}$, where the subscript $R$ denotes the rest frame.

\subsubsection{Degeneracy in Spin}

This is surprising from the point of view of non-relativistic QM. Two spin $\frac{1}{2}$ (or any) states that have the same expectation value of spin projection in one direction have a different one in another! The state is not uniquely given, it seems, by a distribution of spin in one direction. Indeed, the answer is that boosting brings out a degeneracy in the $z$ axis projection of orbital angular momentum, and creates an overlap of states of different orbital quantum number $l$ with the same $m_{z}$ projection. This retains the longitudinal spin projection but changes the transverse projection.

Also, one could speculate whether the reason for a larger transverse angular momentum 
than longitudinal (as will be shown) is that azimuthal symmetry may not hold in a helicity state (in this situation, some partons are moving along faster than others on either side of the central axis, in the $z$ direction). Therefore, there could be degeneracy in a state of definite angular momentum projection, i.e. there could be many states with the same hadron helicity, both in the rest-frame and the fast-moving frame. Thus, two ways of realizing degeneracy in hadron states have been suggested. However, the author does not take the latter too seriously as of now.

When one probes deeper the following important causes for the above results emerge.

\section{1) Boosts Do Not Affect Magnetic Quantum Number, $m$}

When one boosts parallel to the projection of angular momentum (say, orbital), one does not affect the probability density in the transverse (azimuthal) direction(s). The azimuthal dependence goes as $e^{i m \phi}$ before and after the boost. The shrinking/Lorentz contraction of the probability density is due to the fact that now the polar-angle dependence (due to orbital quantum number $l$ ) changes and thus the new wave function is different, as expected. There is no relative phase, however, as $e^{i m \phi}$ is pulled out of the sum:

$e^{i m \phi} \Sigma c_{l} Y_{l}$

where $Y_{l}$ above denotes the polar part of the wave-function. Due to this fact, azimuthal symmetry is retained, and the angular momentum projection still cannot prefer any particular transverse direction. If the boost was transverse to the spin projection, however, the expectation value will change, as stated before.

Further, note that any azimuthally symmetric wave function is necessarily a combination of $m=0$ states since in the absence of other azimuthal dependence, the coefficients of states of non-zero $m$ are given by $\int d \phi e^{i m \phi}=0$, and hence the contribution of states of other $m$ quantum numbers is zero. Moreover, if the wave function is azimuthally symmetric up to a phase, then this means that it is a combination of states of fixed $m$ only with no mutual phase, and is an eigenstate of this value of orbital angular momentum. This applies in field theory as well for all hadrons including the proton. 
So, the claim made earlier can be enhanced: Any state that is azimuthally symmetric up to a phase has no center of mass motion term and is independent under a shift of (longitudinal) origin.

\section{2) Angular Momentum is Translation-Invariant Along Projection Direction}

If one shifts one's origin along the direction of spin projection, the projection does not change. This is easily seen by the fact that $Y_{l}^{m}$ - the angular wave-function- is a function of $\cos (\theta)=\frac{z}{r}$, the polar angle, for eigenstates of $L^{z}$, which has no derivative in the $z$ direction. This means that $Y_{l}^{m}(z)$ and $Y_{l}^{m}\left(z-z_{0}\right)$ will be treated the same way by $L^{z}$ and if one is an eigenstate the other will also be. This can be generally applied to a superposition of states in the "m basis" and the expectation value of $L^{z}$ for any state will be unchanged.

This means that in any order, one can boost and translate along the direction of spin projection and not affect the angular momentum in that direction! This is like the classical case, where a parallel shift of origin would not affect our result in the direction in question. This also explains how experimentalists can, with impunity, measure spin projection of a particle without direct reference to a particular origin, even for a state containing different momenta. What lies at the heart of this observation is the fact that the translation operator (momentum) and the rotation operator (angular momentum) for the same axis commute:

$\left\langle\psi\left|e^{-i a p^{z}} L^{z} e^{i a p^{z}}\right| \psi\right\rangle=\left\langle\psi\left|L^{z}\right| \psi\right\rangle$.

\section{3) Any Shift Works for the Proton}

As shown before, in the case of the proton, any shift of origin for the azimuthally symmetric state will not affect its angular momentum. Granted there are no explicit quantum numbers in QFT, but this fact of independence of shift in origin can be used to identify states that are eigenstates of $L^{z}$. So, for appropriate wave-packets this condition will hold and can be used to probe specific eigenstates of angular momentum for a composite state.

It can be stated here that any state with specific quantum numbers $l, m$ for orbital angular momentum can be written as a wave-packet superposition of momentum eigenstates in NRQM and in QFT (non-relativistically speaking) the same wave packets can be used 
to construct Hilbert states with the same quantum numbers. We say same quantum numbers because the momentum operator translates the state in either theory/affects momentum eigenstates the same way and the expectation value of orbital angular momentum is thus unchanged for the same wave packet. We can use the tools of section 3 to calculate the expectation value of angular momentum for any given wave-packet state.

It is also interesting to note that the hadron can both be an eigenstate of energy and momentum, which is unusual for states in other fully interacting theories. The fact that it has degeneracy in the projection of angular momentum is also not surprising, then, especially as in QCD the orbital nature of the angular momentum of the composite hadron comes to the fore and it stops behaving like an effective elementary fermion of definite spin. One may even question why spin should transform in a way under boosts such that its eigenvalue $\left(s=\frac{1}{2}\right.$ ) overall remains fixed, especially if its transformation properties are similar to orbital angular momentum. However, spin is different from OAM in many counts, including the fact that it is non-zero in the rest frame and does not have a spatially explicit character (it is neither localized nor non-localized as it exists for a spatially diffuse wave function as one single observable quantity. It is an observable by itself that can be thought of initially as being point-like, but ultimately should be thought of as something that makes no reference to the spatial physical characteristics of the particle, but is an independently meaningful physical quantity by itself).

\section{Essential Conclusions Regarding OAM Same in NRQM and QFT}

The non-relativistic QM formalism is certainly different from that of QFT, but when both the refinements of field theory and relativity are added to NRQM, conclusions regarding orbital angular momentum are unaffected. This is shown by the fact that shifting these states in either theory yield the same conclusions, and the essential arguments establishing boost invariance of longitudinal angular momentum are unchanged as well. For example, in a scalar field theory with no spin, $\langle p|\phi(x)| p\rangle$ is more or less treatable as a probability density and the transverse probability density to the boost direction should not change as it is a directly physically observable quantity in a given frame.

As shown in Chapter 2, when one translates the origin of a state, its angular momentum changes by the term: 
$a^{\nu} \int d^{3} p|\phi(p)|^{2}\left\langle p\left|T^{\mu \lambda}(0)\right| p\right\rangle-(\lambda \leftrightarrow \nu)$

This means that if the wave packet is azimuthally symmetric even up to a phase (as all eigenstates of orbital angular momentum, $L^{z}$, are), then the expectation value is unchanged by a shift of (longitudinal) origin! This is a remarkable result. The field theory where this is proven necessarily contains spin via the Dirac spinor and is thus a promotion of the theory of orbital angular momentum, where the same conclusions hold in NRQM, to a theory of azimuthally symmetric wave packets. If the shape of the wave packet in momentum space in field theory is a certain way, then the observables behave the same way as they would for the same shape of packet in NRQM. So, it is the shape of the wave packet (which is unchanged for successive laddered eigenstates of orbital angular momentum in either theory, as momentum operator is responsible for shifting momentum eigenstaes in both cases) that becomes the general concept under consideration. Like traditionally well-behaved dipole moments now, eigenstates of angular momentum resist changing their eigenvalue under translations.

Thus, the core concepts of orbital motion and its conservation are exhibited in the nonrelativistic version of the theory as well, i.e. it is brought out in nature without need for the crutch of relativistic refinement.

Aside:

Can we take the phase arising from rotating an eigenstate of momentum in the $i$ direction about axis $i$, seriously? For a spin $\frac{1}{2}$ particle, this means that to get to the same state, without the phase, one would have to rotate the state by $4 \Pi$. This seems bizarre, then, since rotating the state is equivalent to rotating the frame of reference, and taking a joyful about turn after measuring one's state, only to find that we have altered the phase, is unexpected! This might as well mean that the state can change phase just lying there, at least up to $e^{i \pi}$. If the phase is to be taken seriously, it can be taken seriously only modulo $\pi$. Either that, or there is a higher unphysical dimension where the same point as we see it is actually two different points (!).

Aside (Constants of Motion):

Here is a simple proof of why an operator that commutes with the Hamiltonian does not change in time, i.e. its expectation value is fixed for all states. 


$$
\begin{aligned}
& \left\langle\psi\left|e^{i H t} O e^{-i H t}\right| \psi\right\rangle \\
= & \left\langle\psi\left|e^{i H t} e^{-i H t} O\right| \psi\right\rangle \\
= & \langle\psi|O| \psi\rangle
\end{aligned}
$$

What this means in field theory is that conserved charges, whose time derivatives are zero after integration of charge density over all space, commute with the Hamiltonian. This is why their epectation values do not change in time.

Aside:

If the potential is rotationally symmetric about some axis, then angular momentum about that axis is conserved classically as well as quantum-mechanically. This is be-

cause $L^{z}=-i \frac{\partial}{\partial \phi}$ commutes with the Hamiltonian that is azimuthally symmetric and thus is a constant of motion.

\subsubsection{Pauli-Lubanski Vector: Contribution to Light-Cone Boost or Angular Momentum?}

Yuan and Ji [15] say that $J_{q}$ [2] refers to the total quark transverse angular momentum in a hadron polarized in the same transverse direction. Lorce and Leader[16] disagree and state that it must be longitudinal, since no simple partonic interpretation exists for the transverse case since it is higher twist physics. They also say that the transverse spin sum rule would be frame-dependent due to spin-orbit coupling due to the noncommutation of boosts and rotations, unlike Ji's original sum rule. Let us probe this statement in detail. Since in general, boosts and spin projection direction do not commute, i.e. a boost in general does not leave the spin-projection direction intact in the new frame, a transverse spin rule would presumably make reference to the choice of frame. Ji's $J_{q}$ only makes reference to the second moment of GPDs, which is frame-independent.

The picture is simpler in the case of longitudinal AM (hadron longitudinally polarized), as the angular momentum is unchanged by a boost in the direction along its projection (rotation and boost with reference to the same axis commute). The spin is unchanged 
as we know, and no additional orbital component is added along the direction of the boost. This aspect, too, makes the partonic interpretation more plausible since there would be little difference in spin physics in such a frame of reference from the hadron's rest frame. Just like in the free Dirac theory one easily identifies a piece that resembles orbital motion and a piece that represents intrinsic spin from the total angular momentum operator, one can posit an angular momentum separation more easily in the rest frame of the interacting theory of the hadron- without the mixing of spin and orbital motion due to boosts. At a subtle level, this is really related to the fact that momentum (or orbital motion) and spin transform differently under boosts and thus behave the way non-commuting observables do. They do not just "slide through" the transformation identically.

Traditionally, $P_{q, g}^{+} \equiv \int d x^{-} d^{2} \vec{x} T_{q, g}^{++}$etc. Now, regarding the debate whether it is $J^{+i} \equiv M^{++i}=x^{+} T^{+i}-x^{i} T^{++}$or $J^{-i} \equiv M^{+-i}$ that contributes to transverse angular momentum, Leader and Lorce state that it is the latter, since it is a twist-three object, which is frame-dependent and for which a partonic interpretation is difficult. Thus,

$\left\langle J^{-i}\right\rangle=\int x^{-}\left\langle P_{q}^{i}\right\rangle-x^{i}\left\langle P_{q}^{-}\right\rangle d x^{-} d^{2} \vec{x}$

is the operator that contributes to transverse angular momentum $\left(P_{q}\right.$ is taken to be density here). They state without proof that Ji's original sum rule applies to the longitudinal angular momentum component of quarks in a longtidunally polarized hadron.

However, Ji et al are proposing a whole new way of looking at transverse angular momentum: not via the transverse-spin operator, but by means of the Pauli-Lubanksi vector. In Cartesian coordinates for a state with momentum along $z$,

$$
\begin{aligned}
W_{\mu} & =\frac{1}{2} \varepsilon_{\mu \nu \rho \sigma} p^{\nu} J^{\rho \sigma} \\
\Longrightarrow W_{2} & =p^{3} J^{01}+p^{0} J^{13} \\
& =p^{+} J^{-1}-p^{-} J^{+1}
\end{aligned}
$$

In the second-to-last line, the first operator is instant-form boost and the second, transverse angular momentum. We will show that in taking the expectation value of the 
matrix element, the first does not contribute and we are left precisely with transverse AM. In the last line, the first quantity is the transverse angular momentum operator on the light cone, and the second one is the light-front boost operator, in the quantized theory [16]. It is sufficient to show that the first pair reduce to transverse angular momentum $J^{2}$, and is consistent with taking the expectation value of these operators on the light cone. Let us presently demonstrate this.

$$
\begin{aligned}
J^{01} & \equiv \int M^{001} d^{3} x \\
& =\int d^{3} x x^{0} T^{01}(x)-x^{1} T^{00}(x) \\
& =\lim k \rightarrow 0\left[\frac{\partial}{\partial k^{1}}\left\langle p+k\left|T^{00}(0)\right| p\right\rangle-x^{0} A(0) k^{1} p^{0}\right] \\
& =0
\end{aligned}
$$

Therefore, $W^{2}=E J^{2}$, with even the correct normalization for plane-wave states! The boost operator is defined as a three-dimensional integral consistent with the correct definition of angular momentum in the second term of the Pauli-Lubanski vector. Incidentally, the above proof would have been impossible without using wave packets as a starting point.

The light-front boost multipled by $p^{-}, p^{-} J^{+1}$, is the same order as $p^{+} J^{-1}$ in the PauliLubanski (PL) vector. The PL vector, for azimuthally symmetric states, reduces to tranverse AM along the component chosen. One should be careful when taking Cartesian components of non-covariant operators like $J$ on the light-cone. While we started with $W^{2}$, if we took things straightaway on the light cone, we would get a boost on the light cone in the 1 direction and light-cone angular momentum component from $J^{-1}$. So, one would interpret AM in the 2 direction $\left(J^{2}=W^{2}\right.$, as shown above) as a combination of a boost along the 1 direction and mixed angular momentum components on the light cone. What is the physical use of this? After all, taking $J^{2}\left(J^{3} 1\right)$ on the light cone does not yield the above. What is the physical meaning of these in the context of an operator that is not manifestly covariant, but is expressed as a curl? Why should one take its components on the light-cone directly, if the physical meaning is more manifest in the instant form and unmixed? These are questions that are important to consider. 
Aside: The boost operator is given by $\int d^{3} x J^{0 i}$ (and not $\int d^{4} x$ ) because:

1) It would be unnatural to take all of time into account to construct a instant-form boost.

2) This operator is at the Lagrangian level (pre $\int d t$ ) and in a non-trivial way (via field and momentum densities) contains information about how to change the state upon boosting.

If its time-dependence is explicit, at the end of a physical calculation, it shall not retain a physical meaning by itself. This is because the state transforms according to its projection at a certain time, and certain results may depend on difference in evolution times, but not the time variable itself.

\subsubsection{Good and Bad Components of the Pauli-Lubanski Vector}

The merit of the Pauli-Lubanski vector is that it picks out the "good" component of the spinors only and projects off the bad component. The transverse AM operator, $J^{\perp}$, however does not do this. How, then, are there expectation values the same? This can be seen as follows. Let $|\Psi\rangle=|\Psi\rangle_{g}+|\Psi\rangle_{b}$ be an eigenstate of transverse AM in the rest frame.

$$
\begin{aligned}
W^{\perp}|\Psi\rangle & =W^{\perp}\left(|\Psi\rangle_{g}+|\Psi\rangle_{b}\right) \\
& =a J^{0 \perp}|\Psi\rangle+b J^{\perp}|\Psi\rangle \\
& =\left(A|\Psi\rangle_{g}-d|\Psi\rangle_{b}\right)+\left(c|\Psi\rangle_{g}+d|\Psi\rangle_{b}\right)
\end{aligned}
$$

(In the rest frame $c=d$.) We see above that $W^{\perp}$ has projected out the good component of the state only. However, when the expectation value is taken, the part corresponding to the boost goes to zero for the state in question (in our case the azimuthally symmetric state) and the part that remains and seems to project onto both the good and the bad component is the transverse AM operator. We should remember that we require: 
1) The PL vector should only project out the good component.

2) The general boosted state should not be an eigenstate of $J^{\perp}$.

3) The expectation value of the boost operator alone should vanish.

For all this to be the case:

1) Either we have to be in the rest frame $(c=d)$. But since this is generally true, it must be true that for azimuthally symmetric states, $J^{\perp}$ projects out only the good component of the spinor. Thus $d=0$.

2) The boost operator must annihilate such states, showing that its "eigenvalue" for such states is zero. Thus, $A=0$. So, we claim that the "boost expectation value" (the observable analog of boost, really, like AM is the analog of rotations) should disappear in our state.

\subsubsection{Longitudinal and Transverse Angular Momentum for the $\mathrm{Nu}-$ cleon and the Deuteron}

Ji et al [15] claim that the expectation value of $J^{z}$ in a longitudinally polarized nucleon is of higher-twist than the expectation value of $J^{T}$. Let us calculate these quantities in order and identify the suppression factor in each, while also checking the veracity of the expressions quoted in the literature. First, let us calculate $\left\langle J^{z}\right\rangle$ in a wave-packet state peaked longitudinally. Let us also remind ourselves that we are never in an eigenstate of transverse spin once we boost from the rest frame state of transverse spin projection longitudinally. We use the sum rule developed in Chapter 2:

$$
\begin{aligned}
& \mathbf{M}^{\mu \nu \lambda}(\mathbf{x})=(-\mathbf{i})\left[\frac{\partial}{\partial \mathbf{k}_{\nu}}\left\langle\mathbf{p}_{\mathbf{0}}+\mathbf{k}\left|\mathbf{T}^{\mu \lambda}(\mathbf{0})\right| \mathbf{p}_{\mathbf{0}}\right\rangle-(\lambda \leftrightarrow \nu)\right], \text { so that } \\
& \left\langle p\left|J^{z}\right| p\right\rangle=\frac{\partial}{\partial k^{1}}\left\langle p+k\left|T^{02}(0)\right| p\right\rangle-\frac{\partial}{\partial k^{2}}\left\langle p+k\left|T^{03}(0)\right| p\right\rangle \text { etc. }
\end{aligned}
$$


and using $u_{+}\left(p^{\prime}\right)=\left(1,0,-\frac{\left|p^{\prime}\right|}{E+m} \cos \frac{\theta}{2},-\frac{\left|p^{\prime}\right|}{E+m} \sin \frac{\theta}{2} e^{-i \phi}\right), u_{+}(p)=\left(1,0, \frac{p^{z}}{E+m}, 0\right)$

and $u_{-}(p)=\left(0,1,0, \frac{p^{z}}{E+m}\right)$,

where $\cos \theta=\frac{p^{z}}{|p|}, \cos \phi=\frac{k^{1}}{\sqrt{\left(k^{1}\right)^{2}+\left(k^{2}\right)^{2}}}$ etc. are spherical coordinates describing the momentum $p^{\prime \mu}=\left(E^{\prime}, k^{1}, k^{2}, p^{3}+k^{3}\right)$. Additionally, $p^{\mu}=\left(E, 0,0, p^{3}\right)$, and we obtain up to twist two for the nucleon the following results bearing in mind the twist-two decomposition of the gravitomagnetic form factors for the nucleon given in 2.2.2:

$\left\langle p m_{z}=\frac{1}{2}\left|J^{z}\right| p m_{z}=\frac{1}{2}\right\rangle=0$

In IMF,

$\left\langle p m_{z}=\frac{1}{2}\left|J^{z}\right| p m_{z}=\frac{1}{2}\right\rangle=0$

(We are working in the Dirac basis, so don't need further to account for $p^{0}$ normalization).

$\left\langle p m_{z}=\frac{1}{2}\left|J^{1}\right| p m_{z}=\frac{1}{2}\right\rangle=0$

( + terms involving derivatives in $\mathrm{A}$, which must be zero since by azimuthal symmetry this matrix element must be zero).

$\left\langle p m_{x}=\frac{1}{2}\left|J^{1}\right| p m_{x}=\frac{1}{2}\right\rangle=2 \operatorname{Re}\left\{\left\langle p m_{z}=+\frac{1}{2}\left|J^{1}\right| p m_{z}=-\frac{1}{2}\right\rangle\right\}=2 B(0)$

$\left\langle p m_{y}=\frac{1}{2}\left|J^{2}\right| p m_{y}=\frac{1}{2}\right\rangle=2 B(0)$ (by natural extension of above)

$\left\langle p m_{y}=\frac{1}{2}\left|J^{1}\right| p m_{y}=\frac{1}{2}\right\rangle=5 A(0)+4 B(0)(\mathrm{IMF})$

(Same leading term as previous matrix element- interesting) 
Notes:

$\left|m_{x}=\frac{1}{2}\right\rangle \equiv|+\rangle+|-\rangle$ etc

$\left|m_{y}=\frac{1}{2}\right\rangle \equiv|+\rangle+i|-\rangle$ etc

It seems that the derivative of the structure functions should be zero with respect to transverse momenta at $\Delta^{2}=0$.

It seems that the longitudinal angular momentum is indeed relatively suppressed by $p^{+}$ compared to transverse AM, as claimed by Ji et al [15]. We should ignore one factor of $E$ or $p^{+}$anyway since it is from the normalization of the spinors. So, when we introduce a factor of $\frac{1}{p^{+}}$for dimensional correctness, we see above that $J^{T}$ survives, but $J^{z}$ does not. And $J^{T}$ is frame-independent in our result in the IMF (goes as $B(0) / m$ ). The exact form of the result for transverse AM differs from Ji's evaluation of the Pauli-Lubanski vector, though.

Aside:

In preparing the wave-packet state, one can include arbitrarily small contributions from transverse momentum components, $\vec{k}$, in the mostly longitudinal wave-packet one generally uses in the spin sum rule. When taking the derivative with respect to $k^{2}$ at $k^{2}=0$, when $k^{1}$ in the nucleon spinor is non-zero yet very small, there is an ambiguity as to which of the two variables should be taken to be smaller, i.e. taken to zero first. In the usual definition of a derivative, this question does not come up since the constants of differentiation are finite quantities that are usually not infinitesimally small, and if they are meant to be smaller than the parameter varied in taking the derivative, they can be taken to be zero.

For us, the varied (derivative) paramter $\left(k_{x}, k_{y}\right.$ etc) is really independent of the wavepacket paramter $\left(k_{x}, k_{y}\right.$ etc) and to avoid all ambiguity the varied parameter is taken to be arbitrarily small while the other is fixed to be small. For example, if $k_{x}$ is the varied paramter, the derivative is calculated at $k_{x}=0$, and $k_{y}$ is allowed to be slightly larger.

Deuteron (IMF): 


$$
\begin{aligned}
& \left\langle p m_{z}=+1\left|J^{z}\right| p m_{z}=+1\right\rangle=6 \sqrt{2} i \frac{\left(p^{+}\right)^{2}}{m} G_{5}(0) \\
& \left\langle p m_{x}=+1\left|J^{x}\right| p m_{x}=+1\right\rangle=-\frac{1}{4 m^{2}}\left(p^{+}\right)^{3}\left(G_{5}(0)+G_{1}(0)\right)+\text { H.O. }
\end{aligned}
$$

This gives us the important result that transverse angular momentum is dominant over the longitudinal in both the nucleon as well as in the deuteron.

Notes:

Only the relative order of $p^{+}$in the different expectation values is important just now. Accounting for the extra $P^{0}$ in normalization will drop the result down one order in momentum $p^{+}$.

The Lorentz Transformation Matrix used for boosting the deuteron state (which takes us to a state of large longitudinal momentum $p^{z}$ and small transverse momenta $k^{x}, k^{y}$ ) is

$$
\Lambda_{v}^{v^{\prime}}=\left[\begin{array}{cccc}
\gamma & -\frac{k^{x}}{m} & -\frac{k^{y}}{m} & -\frac{p^{z}}{m} \\
-\gamma \frac{k^{x}}{m} & 1+(\gamma-1) \frac{\left(k^{x}\right)^{2}}{p^{2}} & (\gamma-1) \frac{k^{x} k^{y}}{p^{2}} & (\gamma-1) \frac{k^{x} p^{z}}{p^{2}} \\
-\gamma \frac{k^{y}}{m} & (\gamma-1) \frac{k^{x} k^{y}}{p^{2}} & 1+(\gamma-1) \frac{\left(k^{y}\right)^{2}}{p^{2}} & (\gamma-1) \frac{k^{y} p^{z}}{p^{2}} \\
-\gamma \frac{p^{z}}{m} & (\gamma-1) \frac{k^{x} p^{z}}{p^{2}} & (\gamma-1) \frac{k^{y} p^{z}}{p^{2}} & 1+(\gamma-1) \frac{\left(p^{z}\right)^{2}}{p^{2}}
\end{array}\right]
$$

where $p^{2} \equiv\left(k^{x}\right)^{2}+\left(k^{y}\right)^{2}+\left(p^{z}\right)^{2}$.

It is worth asking here why a deeper/general separation (beyond leading twist) of the different OAM contributions should at all be plausible. There seem to be no deeper reasons to justify it, as stated before. In a sense, it is like asking what the contribution of the molecular elements of water and the liquidity of their phase is individually to its wetness. In classical field treatments, one may sometimes be able to separate the momentum of electric fields and electrons. For the momentum sum rule one can separate the contributions since a probabilistic momentum-based model of PDFs is available and one may do this for quarks up to leading twist only. But no such model exists for angular momentum overall (since PDFs are, already, an expected "value" or representation of the theory, one cannot get the expected angular momentum decomposition from them) and other effects need to be taken into account. Besides, if there is a partonic interpretation only up to twist two, and they are otherwise inherently entangled at deeper levels 
of the theory, then why should one insist on separate gauge invariance of the operators or their individual expectation values?

\subsubsection{What About Orbital Angular Momentum?}

Polyakov et al [17] show that $L^{z}=-\int d x G_{3}(x) x=\int d x x(H(x)+E(x))-\int d x \tilde{H}(x)$, where $G_{3}$ is the twist-3 GPD that appears first in the transverse correlator. This shows that orbital angular momentum is a subleading twist operator longitudinally, since there is no explicit factor of $P^{+}$befitting a spin-2 (2 Lorentz indices) operator. Yet, it can be expressed as a moment of a twist-3 GPD.

The transverse analog is $L^{\perp}=P^{+} \int d x(H+E) x-\int d x g^{T}$. There is no corresponding sum rule for this outlined in the literature and seems highly unlikely given the mixing of twists/order of $P^{+}$above. Note that $g^{T}$ is the forward limit of a twist-3 GPD.

\subsubsection{What Does a Boost Do Classically and Quantum Mechanically to Angular Momentum?}

Imagine a classical current-carrying loop with dipole moment (angular momentum) along $\hat{z}$. If one boosts along the $z$-axis, there is no change in the $z$-projection of the orbital angular momentum of the current, but a contribution in the transverse direction is added. The first claim is seen if one chooses an origin that lies at the center of the loop in the new frame. As transverse directions are not affected by the boost, the width of the loop remains the same and the only change in momentum can be in the $z$ direction for points around the loop. At any given time in the new frame, the particles in the loop will be at the same relative transverse position as in the old frame, with the same transverse momenta. The current in the new frame will simultaneously form a circular loop again, as all points/currents on the loop will lie at the same position on the $z$ axis in the new frame, and thus occur simultaneously as well. In the quantum mechanical analog, as parallel boosts do not affect angular momentum, one may suppose that its projection in this direction as well as in the transverse direction must remain the same. However, this is not the case and is addressed in another section.

Imagine now the current loop having angular momentum in the transverse direction only, say $x$. A longitudinal boost (along $z$ ) will now shrink its orbital contribution in 


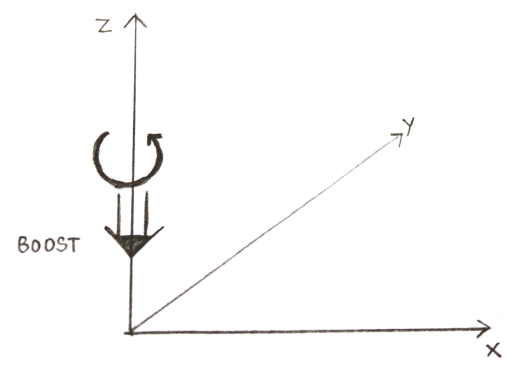

FiguRE 4.1: Parallel/Longitudinal Boost on current loop carrying angular momentum along $\hat{z}$

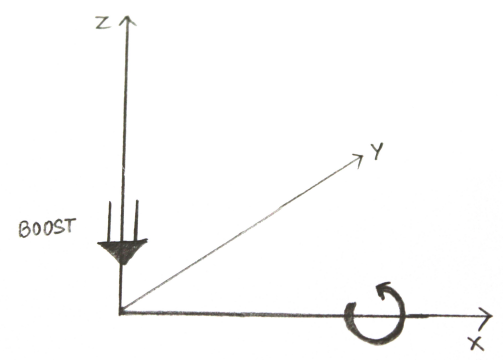

FiguRE 4.2: Transverse (along $-z$ ) boost on current loop carrying angular momentum along $\hat{x}$

the $x$ direction, provided we use a moving origin that again remains at the center of the loop. Otherwise, it is arbitrary what origin we are referring to. A back-of-the-envelope calculation reveals that the effect of increasing momentum is countered heavily by the Lorentz contraction of the loop ( by order $\gamma$ ). In the QCD analog, one can ask how exactly a relativistic boost would affect the spin projection of the particle. One possiblity is that it shrinks to 0 like in the classical case, i.e. its distribution of spin up and down becomes uniform in the transverse direction. This would also make it blind to whether originally we started in an $m_{l}=+1$ or $m_{l}=-1$ angular momentum state, as the loop (particle wave function) would be highly shrunk now. Another possibility is that it changes it in a frame-dependent way. We shall explore this in the following sections.

In the case of a point-like particle, which would presumably behave more like the classical analog, these effects are seen. The transverse expectation value of spin shrinks to 0 in the infinite momentum frame, as the contribution of the GPD $H(x, 0,0)$ (unpolarized PDF $\left.F_{1}(x)\right)$ disappears, as seen in the earlier sections. The contribution of $E(x, 0,0)$ 
is always absent in the structure of a point-like particle. So, $E(x, 0,0)$ represents deviations in the field theory of hadrons from their classical/quantum counterparts. For a scalar field, there would be less of a difference from the classical picture as well. It seems here that a particle moving very fast tends towards being a helicity eigenstate, even if it did not start off this way, and this tendency is exhibited seemingly above by the fact that the transverse spin distribution is uniform and thus projects out spin along the positive $z$-axis. One might speculate that just as very fast-moving particles fall into a helicity eigenstate like massless particles and conserve the chiral current, by plausible extension perhaps point-like particles in QCD tend towards acquiring characteristics helicity eigenstates. However, this conclusion is negated by the commutation of $J^{z}$ and boosts along $z$, fixing the expectation value of $z$ (longitudinal) angular momentum.

In the previous chapter, it was shown that the contributions of the GPD $E$ cancel for up and down quarks when summing their total angular momentum. This will happen for both longitudinal and transverse angular momentum, then, since $E^{u}$ and $E^{d}$ appear with the same coefficients in the angular momentum sum rule. So, the classical heuristic argument above would make a lot of sense if the contribution of longitudinal and transverse angular momentum disappears up to twist two altogether. This would happen if the contribution of the GPD $H$ is anyway subleading in the sum rule.

Aside:

However, orbital angular momentum (and spin) is a subtly manifest quantity in the quantum picture. As position and momentum cannot be simultaneously known or zoned into, in position or momentum space, it is one order subtler than the position-space or momentum-space eigenvalue distribution. Therefore, it is also possible a boost may not affect it as one may think from a classical standpoint. Spin is even subtler than OAM since there is no explicit spatial wave function that gives rise to the magnetic dipole moment that it is. It transforms according to its intrinsic value of $s$, and is non-zero even in the rest frame, unlike OAM which is zero in the rest frame and is not constrained to any intrinsic maximum value. Spin is not a spatially localized concept or one that makes reference to space, and so it transforms differently from both classical angular momentum and orbital angular momentum in QM. It exists in a spatially non-localized state as a fixed observable quantity, yet for purpose of understanding one can begin by thinking of spin as an infinitesimally small current loop with a fixed magnetic dipole moment at a given point.

\section{When Does a Boost Affect Angular Momentum?}


It is obvious by azimuthal symmetry that in a helicity state, the exepctation value of transverse angular momentum will be 0, i.e. $\left\langle p m_{z}\left|J^{x}\right| p m_{z}\right\rangle=0$.

Let us now consider the expectation value of longitudinal angular momentum in a state which would be transversely polarized in the rest frame. $\Lambda$ will be the matrix that boosts along the $-\hat{z}$ direction to put us in the infinite momentum frame. Working in the $z$-basis as usual, and up to normalization constants,

$$
\begin{aligned}
\left\langle\Lambda \xi_{\hat{x}}\left|J^{z}\right| \Lambda \xi_{\hat{x}}\right\rangle & =\left\langle\Lambda \uparrow\left|+\left\langle\Lambda \downarrow\left(J^{z}\right) \mid \Lambda \uparrow\right\rangle+\right| \Lambda \downarrow\right\rangle \\
& =\left\langle p m_{z}=1\left|J^{z}\right| p m_{z}=1\right\rangle+\left\langle p m_{z}=-1\left|J^{x}\right| p m_{z}=-1\right\rangle \\
& +\left\langle p m_{z}=-1\left|J^{z}\right| p m_{z}=1\right\rangle+\left\langle p m_{z}=1\left|J^{z}\right| p m_{z}=-1\right\rangle \\
& =\frac{1}{2}-\frac{1}{2}+2 \operatorname{Re}\left\{\left\langle p m_{z}=1 \mid p m_{z}=-1\right\rangle\right\} \\
& =0
\end{aligned}
$$

Thus, we have shown that a longitudinal $(-\hat{z})$ boost does not affect the expectation values of angular momentum in certain situations. It does not affect the transverse or longitudinal angular momentum expectation value in a helicity eigenstate, and it does not affect the longitudinal expectation value in any state.

$$
\begin{aligned}
\left\langle\Lambda \xi_{\hat{x}}\left|J^{x}\right| \Lambda \xi_{\hat{x}}\right\rangle & =\left\langle\Lambda \uparrow\left|+\left\langle\Lambda \downarrow\left(J^{x}\right) \mid \Lambda \uparrow\right\rangle+\right| \Lambda \downarrow\right\rangle \\
& =\left\langle p m_{z}=1\left|J^{x}\right| p m_{z}=1\right\rangle+\left\langle p m_{z}=-1\left|J^{x}\right| p m_{z}=-1\right\rangle \\
& +\left\langle p m_{z}=-1\left|J^{x}\right| p m_{z}=1\right\rangle+\left\langle p m_{z}=1\left|J^{x}\right| p m_{z}=-1\right\rangle \\
& =2 \operatorname{Re}\left\{\left\langle p m_{z}=1\left|J^{x}\right| p m_{z}=-1\right\rangle\right\} \\
& =2 \operatorname{Re}\left\{(-i)\left\langle p m_{z}=1\left|J^{y}\right| p m_{z}=-1\right\rangle\right\}
\end{aligned}
$$

In going to the last line, we have used $J^{x}=i\left[J^{y}, J^{z}\right]$ and the hermiticity of these operators.

A boost should affect a state in a homogeneous way if it was homogeneous in all of space to begin with. That is, the contributions of the boost at different "points" to 
angular momentum in the new frame should not be different in such a state. A scalar field wave-function of a momentum eigenstate is a simple example. In such states, the expectation value of orbital angular momentum seems unaffected by a boost, as this would yield a different momentum eigenstate that is homoegeneous in space (see figure below). Similarly, for a spin $\frac{1}{2}$ particle, one can boost the spinor using the appropriate representation of the Lorentz group, and we would end up with a different momentum eigenstate- which would be homogeneous in space. When one integrates the "pure orbital part," (however defined) in principle, over all space, each coordinate has a negative analog that cancels its contribution, the momentum eigenvalue being held the same the whole time.

Also, again considering only orbital effects in principle, when a state that is azimuthally symmetric is boosted along $-\hat{z}$, the angular momentum would not change in the $z$ direction. This is also due to the homogeneity in space of the state before the boost, and hence if "all points are affected the same way," there is no change in AM when we integrate over all space. In fact, in this particular example, there is no orbital AM $(m=0)$ as the state is azimuthally symmetric.

Aside:

Incidentally, the canonical commutation relation between OAM operators, $L^{i}$ would no longer hold for plane wave states (definition of $\vec{L}$ is of course now a matter of debate, yet this is a real problem if the concept of orbital angular momentum is to be defined at all) since they would be simultaneous eigenstates of $L^{x}, L^{y}$ and $L^{z}$, another indication that plane wave states cannot be taken seriously by themselves. This is obvious if we consider the proton in the rest frame, where there is no extrinsic directional bias. One simultaneously knows that the OAM in different directions is zero. $[x, p]$ can not be simultaneously known but $\left[L^{x}, L^{y}\right]$ can be in this case, as the latter are functions of both position and momentum. Again, this shows that angular momentum is one order subtler than position and momentum.

Also, such a state does not behave correctly under boosts. For example, a scalar field boosted along the direction in which the momentum is zero would yield zero momentum in this direction even after the boost pictorially, as the Lorentz contraction of a straight line wave-function is still a straight line! 


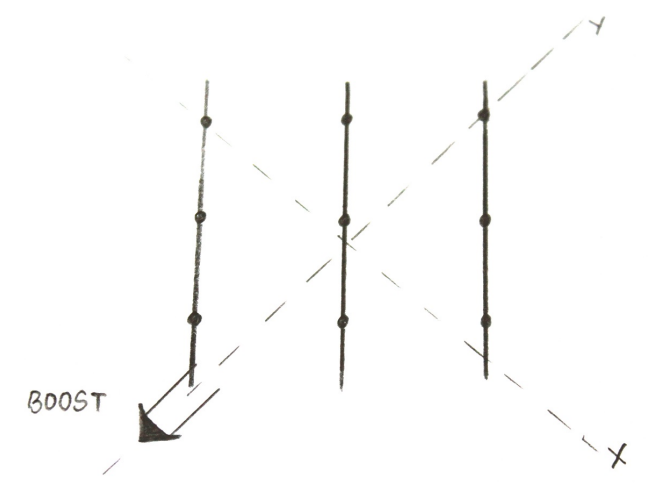

FiguRE 4.3: Boosting homogeneous scalar field of fixed momentum

Aside:

A multipole expansion can be done on the expectation value of the field $A^{\mu}(x)$ in powers of $\frac{1}{r}$, the distance from the origin being $r$, to separate out its monopole, dipole, quadrupole contributions just like classically. This can be done for any quantum field theory independently of its equations of motion. This will enable us to pick out matrix elements that appear in general form as coefficients of these powers of $\frac{1}{r}$ as monopole, dipole etc. based on simply the expectation value of the field throughout space. This would be the quantum version of the multipole expansion.

Aside: How does one distinguish a zero-eigenvalue state from the fact that the state is merely annihilated by the operator? One makes the ansatz that if the operator represents an observable (as opposed to a ladder/creation operator etc.), then it yields identically a 0 eigenvalue when sandwiched between instances of this state.

\subsubsection{Meaning of Spin and Orbital Contributions in a Stationary State}

Orbital Angular Momentum lies phenomenologically beyond the parton model (where we do not consider transverse momentum of the quarks, $k_{T}$ ). This is how the "spin crisis" got its name, since one expected all the spin of the proton to come from parton spins, as there can be no longitudinal partonic OAM from the parton model. This has been motivated further in Chapter 2 with the consideration of corresponding quantum mechanical states to the proton of zero OAM. It is important to note that the angular 
momentum sum rule relies on twist-2 GPDs, some of which have a direct partonic interpretation in the forward limit. Though the parton model accommodates/is related to the total angular momentum operator via the operator product expansion (OPE), it is not a good model for probing angular momentum distribution. The parton model accommodates large collinear partonic momentum and fixes the parton spin to determine the scattering cross-section. It is a good model for cross-section data, but not for orbital angular momentum. If it were, the sum of the spins of the valence quarks would give the angular momentum of the proton, but that is far from the case (less than $\frac{1}{2}$ )! Even when one interprets $H$ and $E$ via helicity amplitudes $A_{\Lambda^{\prime} \lambda^{\prime} \Lambda \lambda}$ [4], only the spin-flip of the quarks comes into question, not their OAM, though this is one level deeper than the first-order partonic interpretation.

One makes the ansatz that the spin represented by spin PDFs retain their meaning when we talk about spin and orbital content making up the total angular momentum of the hadron. After all, there is $k_{T}$ (orbital motion/coupling possiblities etc) in the quarks to second order. So, let us take note of the subtle entry that the magnetic moment, $G_{M}$, makes in the sum rule. Since quarks are really indistinguishable particles and are entangled, spin PDFs represent only a first order treatment. The fact that their dipole-moment contribution, $G_{M}$, describes the total hadron spin means that there is entanglement between these fermions which leads to the dipole-dipole interaction from the interaction Hamiltonian figuring in the spin content. Let us remind ourselves that $G_{M}=2\left(F_{1}(0)+F_{2}(0)\right)$, a combination of the Dirac and Pauli form factors. It is also a factor that determines the strength of $\vec{\mu}$, the dipole moment of the fermion, which couples to the magnetic field potential in scatering.

For a stationary state, the interaction term would not enter unless there was coupling between the various degrees of freedom. If the spins were independent, this term would not enter, precise and unaltered in $J$. One may argue that this would be true for the electron as well- that $G_{M}$ would describe its angular momentum. This is true because the decomposition of the EMT would be the same for the electron as it is for QCD up to twist 2, and the OPE involving twist-2 GPDs would also be the same, as no explicit $Q^{2}, q$ dependence enters in these terms. In this case, $G_{M}$ would represent spin-orbit coupling. The hadron can thus be thought of as many independent quarks with spinorbit coupling adding up to give the total angular momentum or one can infer from the presence of the magnetic form factor that there would be coupling between the various quarks at a deeper level. This term can only possibly come from coupling between the partons, showing that their angular momentum has non-separability (as well as separability) built in. In fact, it shows that the "dark" OAM was always there (via this 
coupling) and it is not just the individual spins adding up to give the total spin of the proton.

The proton is a stationary state. From what we have seen from inelastic scattering experiments, the proton seems not to have much degeneracy. The quarks, at least, are identical particles and in principle antizymmetrized overall, explaining this fact. This means that expectation values of observable quantities, including angular momentum and spin, should not change in time. However, this does not mean that they are not entangled. The partons need reference to to each other's angular momentum content to define their own, in principle. Beyond first order, one starts to appreciate this more when probing the proton spin sum rule.

\subsubsection{Why Transverse Angular Momentum Dominates}

It seems physically very reasonable that transverse angular momentum dominates its longitudinal counterpart for both the proton and the deuteron. It is one order higher in $P^{+}$in the infinite momentum frame. If one views the hadron moving very fast in this frame, and the partons moving in a collinear fashion in the same direction with limited transverse momentum, then one sees that it is much easier for them to have partial/complete orbits about the transverse axis than the longitudinal axis. One way in which this is seen is that the longitudinal component that needs to be large is already fixed by the hadron and the error associated with the exactness of this large momentum gives us transverse angular momentum. Ignoring azimuthal symmetry for the moment, boosting to a frame that is the center of momentum for the fast-moving quarks, one would see quarks moving with substantial momenta in opposite directions longitudinally, giving large transverse angular momentum. For obtaining large longitudinal angular momentum, one would require both transverse momenta $\left(k_{x}, k_{y}\right)$ to be substantial. However, it is the subtantial longitudinal momentum near the central transverse axis of the proton/hadron that leads to $J^{\perp}\left(T^{03}\right)$ be of leading order and $J^{z}\left(T^{0 \perp}\right)$ be subleading.

All of this said, it does seem unlikely that there would be a degeneracy associated with what should be an azimuthally symmetric state about the $z$ axis: the hadron moving along in the IMF.

One could attempt here to guess which helicity amplitudes should describe longitudinal and transverse angular momentum expectation values, just based on intuition about 
spin. Then, one can later compare them to the actual helicity amplitudes that appear in the expectation value. It is a reasonable guess that the partonic propensity to flip spin must be related to transverse partonic angular momentum.

\subsubsection{Angular Momentum Contribution of the Magnetic Form Factor for the Proton and Deuteron}

Let us explore how angular momentum is related to the magnetic form factor, $G_{M}$, in the case of either hadron. A priori, one would guess them to be closely related since the dipole moment of a particle of state would naturally be related to its scattering properties off a magnetic field. In the case of the proton, supposedly, for a longitudinally polarized state:

$J^{z}=\frac{1}{2} \int d x x(H(x, o, o)+E(x, 0,0))$,

where $\int d x H(x, 0,0)=F_{1}(0), \int d x E(x, 0,0)=F_{2}(0)$ and $G_{M}=2\left(F_{1}(0)+2 F_{2}(0)\right)$.

For the deuteron, again longitudinally polarized:

$J^{z}=\frac{1}{2} \int d x x\left(H_{2}(x, 0,0)\right)$,

$\int d x H_{2}(x, 0,0)=G_{M(d)}$

This is what we would expect. For both hadrons, the behavior of the form factor is the same. The longitudinal angular momentum is essentially given by moments of the GPDs defining the magnetic form factor in either case, when a longitudinally polarized state is chosen. However, this is not the case for transverse angular momentum in a transversely polarized state (boosted from rest-frame transverse spin projection), as shown in the second section. These contributions are somehow hidden in the longitudinal case. This difference needs to be explained, as the classical magnetic field chosen to derive this form factor as the main contributor to the scattering cross-section did not have a preferred direction. But when calculating angular momentum, it directly enters only in the longitudinal expectation value and not the transverse one, for both the proton and the deuteron. 


\subsubsection{What Does Boosting Do to Spin Individually?}

We ask the question: what happens to the expectation value of OAM and spin if one boosts transverse to the angular momentum projection from 1) the rest frame? 2) the frame boosted parallel to the angular momentum projection? In both these frames, prior to the transverse boost, the angular momentum is the same. It is clear that since transverse boosts do not commute, the momentum of the final state would depend on the initial. Hence, OAM would be different in the two final cases above. One might posit, however, that spin, being intrinsic, and should be affected the same way by the transverse boost in the two cases. The answer is that it is affected differently in the two cases. Intrinsic motion is affected by explicit boosts, just like OAM is. Even though OAM does not change (about the appropriate moving origin, of course) in the direction parallel to the boost, the character of this boost does determine how a subsequent boost will affect OAM. In QED, this is seen with the canonical spinor (e.g. Peskin and Schroeder's standard field theory textbook), where the two-component spinor does not transform linearly under boosts. It is easy to imagine why this would hold true in the more complicated case of strong interactions.

The spinor reads:

$u(p)=(\sqrt{p . \sigma} \xi, \sqrt{p . \bar{\sigma}} \xi)$,

where $\xi$ is the two-component spin projection in the rest frame. It is easy to see, therefore, that a transverse boost affects the spin projection in a way that is dependent on the initial longitudinal momentum.

\subsubsection{Ji's Recent Argument for Gluon Spin}

$\left\langle\left|S^{z}\right|\right\rangle$ has a meaning in all frames. For pure Abelian gauge theory, the photon spin is given by $E^{\perp} \times A^{\perp}$. This same expression would hold for the gluon as well, canonically. X.Ji notes that in the $A^{+}=0$ gauge, gluon spin $\Delta G$ is equal to $E^{\perp} \times A^{\perp}$ [18]. The latter expression is not gauge-invariant. For longitudinal spin to equal the above value in pure gauge theory in every frame is non-trivial. For the cross-product to be unchanged under longitudinal boosts is also non-trivial, as $E^{\perp}$ changes under longitudinal boosts. However, Ji's argument is that it is highly non-trivial for this to occur in the fully interacting theory, unless $E^{\perp}$ were an independent observable, with specific transformation 
properties. It is generally not, as under a Lorentz transformation, the meaning of the perpendicular field component is not individually retained; there is inherent mixing with the other components. There is no "test charge" that responds differently to the perpendicular component. Only in the infinite momentum frame does $E^{\perp}$ begin to behave like an independent observable, as a (longitudinal -here all boosts are longitudinal) boost here can only change this component of $E$ in a standard universal way, according to the Lorentz transformation properties under such a boost. ( $E^{z}$ is unchanged, of course.) So, he says that gluon spin does have a physically relevant meaning in the IMF and in the $A^{+}=0$ gauge.

\subsubsection{Spin-Orbit Coupling}

There are three principal categories in which one can view motion-spin coupling:

1) OAM/motion does not affect spin. Example: boosting to (viewing in) a frame moving parallel to spin projection.

2) OAM affects spin but statistics do not change in time. Example: stationary state of a Hamiltonian involving spin-orbit coupling.

3) OAM affects spin in a way that statistics change in time (not a stationary state). In quantum field theory, though, conservation laws dictate that total angular momentum is a constant of motion. Similarly in non-relativistic QM as well, the OAM and spin operators commute with the Hamiltonian, at least one without angular momentum coupling of any kind.

Incidentally, a fourth category, related to the second, would be the case of viewing the same situation in a different reference frame. Example: a very slow incremental (adiabatic) boost in any direction, and then leaving the state be. In time its expectation values will settle to become time-independent, once all reference to the frame- external to the Hamiltonian- has subsided. Also, there is a subtlety in the first point, as shown above. The longitudinal boost will not explicitly affect the spin, but will pave the way for the way a transverse boost will affect it. 


\subsubsection{Is There Origin Dependence in Angular Momentum? (On the Density Interpretation)}

Let us ask the question whether there is any bias/reference to a particular origin in treating the matrix element of the EMT at a particular space-time point (Belinfante or otherwise). This is a natural question since we formulated our sum rule in momentum space, which has no reference to position in the state itself. Some thought would make one question why a seemingly homogeneous state (up to a phase that has no Hilbert-space dependence) would evaluate to different densities at different points in space without any a priori reason. After all, this difference is not just a relative translation phase between the two points, but a different physical value of the momentum contained in the fields in these distant pockets of space. This is seen when the diverse phases are integrated, their difference is only due to the separation distance between two points.

$$
\int \phi^{*}\left(p^{\prime}\right) \phi(p)\left\langle p^{\prime}\left|T^{\mu \nu}(a)\right| p\right\rangle=\int \phi^{*}\left(p^{\prime}\right) \phi(p) e^{i \Delta a}\left\langle p^{\prime}\left|T^{\mu \nu}(0)\right| p\right\rangle
$$

When one constructs a wave-packet peaked in momentum space about some momentum $p_{0}$, one gets a small origin-dependent term (this was shown before). Practically speaking, in an experiment, one creates this condition of a peaked momentum space distribution even in a physically small region. This is due to natural constants enabling such convenient scaling on both sides, when ones goes from a peaked Gaussian in Fourier (momentum) space (having a plane-wave representation in position space) to an off-peak Gaussian (having a relatively localized position).

\subsubsection{Covariance of the Off-forward Matrix Element}

A question was raised in the literature as to the correctness of the off-forward covariant form of the tensor decomposition $\left\langle p^{\prime}\left|T^{\mu \nu}\right| p\right\rangle$. This objection is not substantial. This form is covariant for the same reason that the Dirac equation is covariant. Lorentz transforming on the indices $\mu, \nu$ above: 


$$
\begin{aligned}
\Lambda_{\mu}^{\mu^{\prime}} \Lambda_{\nu}^{\nu^{\prime}}\left\langle p^{\prime}\left|T^{\mu \nu}\right| p\right\rangle & =\bar{u}\left(p^{\prime}\right) \Lambda_{\mu}^{\mu^{\prime}} p^{\mu} \Lambda_{\nu}^{\nu^{\prime}} \gamma^{\nu} u(p) \\
& =\bar{u}\left(p^{\prime}\right) \Lambda_{\mu}^{\mu^{\prime}} p^{\mu} \Lambda^{-\frac{1}{2}} \gamma^{\nu} \Lambda^{\frac{1}{2}} u(p) \\
& =\bar{u}\left(\bar{p}^{\prime}\right) \Lambda_{\mu}^{\mu^{\prime}} p^{\mu} \gamma^{\nu} \Lambda^{\frac{1}{2}} u(p) \\
& =\bar{u}\left(\bar{p}^{\prime}\right) \Lambda_{\mu}^{\mu^{\prime}} p^{\mu} \gamma^{\nu} u(\bar{p}),
\end{aligned}
$$

which is nothing but the same operator (the energy-momentum tensor) evaluated in the boosted frame. Thus, the form is correct. If there was a lower index involved, one would use the same arguments as above after rewriting $\gamma_{\nu}=g_{\mu \nu} \gamma^{\mu}$ and contracting with the metric tensor $g_{\mu \nu}$ at the end.

That form is covariant and the off-forward spinor product $\bar{u}\left(q^{\prime}\right) u(q)=\left(E_{q}^{\prime} E_{q}\right)^{\frac{1}{2}}$ is invariant. Of course. if one implements Lorentz transformations correctly on the spinors in going to a different frame one does not both change the momentum variable of the spinor and transform the spinor with $\Lambda_{f} r a c 12$ : one just does the latter (the Dirac wavefunction transforms differently from its bare Fourier component, the Dirac eigenspinor). Keeping this in mind, the above form is physically meaningful and robust, as the momenta transform correctly as four vectors.

\subsubsection{Aside: Operator-shifting in Field Theory}

One may suppose that shifting in operator algebra is as simple as follows. Say we have a state defined by $|\psi\rangle=T^{\mu \nu}(x)\left|\psi_{0}\right\rangle$. We then shift the operand (ket) by an amount $a^{v}$ to the left in position space, and then we act with the same operator at the space-time point $x^{v}-a^{v}$, seemingly to preserve the original result. That is, one may think

$|\psi\rangle=T^{\mu \nu}(x)\left|\psi_{0}\right\rangle=T^{\mu \nu}(x-a)\left|e^{i P . a} \psi_{0}\right\rangle$

where $P^{\mu}$ is the momentum operator. However, would the expectation value also not be constrained to be the same if we shifted the state by that amount but also evaluated the operator at the point where the original field orientation would now "be"? After all, the expectation value is a physical quantity devoid of phases and would have to be the 
same. Thus,

$\left\langle\psi_{0}\left|T^{\mu \nu}(x)\right| \psi_{0}\right\rangle=\left\langle\psi_{0} e^{-i . P . a}\left|T^{\mu \nu}(x-a)\right| e^{i . P . a} \psi_{0}\right\rangle$

This implies that

$\left\langle\psi_{0}\left|T^{\mu \nu}(x-a)\right| e^{i P . a} \psi_{0}\right\rangle=\left\langle\psi_{0} e^{-i P . a}\left|T^{\mu \nu}(x-a)\right| e^{i P . a} \psi_{0}\right\rangle$

which clearly cannot be true. In fact, one can see how if one used wave packets to calculate the above matrix elements in the momentum basis one would end up with an extra phase factor of $e^{i p . a}$ integrated over all values of $p^{i}$ in the RHS, for arbitrary $\phi(p)$, when compared to the LHS, in the equation above.

This shows that shifting an operator in space is like shifting in time- it takes into account both the "bra" and the "ket" a priori, like in the Heisenberg picture where the operator is shifted in time from both sides.

\subsubsection{Comparing the Ji and JM Decompositions of the EMT}

Let us try to compare to see if there is any resemblance between the decompositions given by JM [5] and that by $\mathrm{Ji}[2]$ for the expectation value of the EMT at $x=0$, $\left\langle p^{\prime}\left|T^{\mu \nu}(0)\right| p\right\rangle$, for the proton. In particular, since there are only two terms in JM's decomposition, let us see how they may compare to the first two terms in Ji's decomposition. JM write:

$\left\langle p^{\prime}\left|T^{\mu \nu}(0)\right| p\right\rangle=A_{0}\left(\Delta^{2}\right)\left(p+p^{\prime}\right)_{\mu}\left(p+p^{\prime}\right)_{\nu}+A_{1}\left(\Delta^{2}\right) i\left(\varepsilon_{\mu \alpha \beta \sigma}\left(p+p^{\prime}\right)_{\nu}+\varepsilon_{\nu \alpha \beta \gamma}\left(p+p^{\prime}\right)_{\mu}\right) \Delta^{\alpha}\left(p+p^{\prime}\right)^{\beta} s^{\sigma}$

where $s^{\sigma}$ is the traditional spin-vector with the expectation value of spin-projection noted in the spatial components. It has already been argued that the above decomposition cannot be covariant [19]. Ji writes his with Dirac spinors for the proton thus, making it explicit in his notation that the decomposition is valid for both quarks and gluons [2]: 


$$
\begin{aligned}
\left\langle p^{\prime}\left|T^{\mu \nu}(0)\right| p\right\rangle & =\bar{u}\left(p^{\prime}\right)\left[A_{q, g}\left(\Delta^{2}\right)\left(\gamma^{\mu}\left(p+p^{\prime}\right)^{\nu}+\gamma^{\nu}\left(p+p^{\prime}\right)^{\mu}\right)+i B_{q, g}\left(\Delta^{2}\right)\left(\left(p+p^{\prime}\right)^{\mu} \sigma^{\nu \alpha}\right.\right. \\
& \left.+\left(p+p^{\prime}\right)^{\nu} \sigma^{\mu \alpha}\right) \Delta_{\alpha} \frac{1}{2 M}+C_{q, g}\left(\Delta^{2}\right)\left(\Delta^{\mu} \Delta^{\nu}-g^{\mu \nu} \Delta^{2}\right) \frac{1}{M} \\
& \left.+\bar{C}_{q, g}\left(\Delta^{2}\right) g^{\mu \nu} M\right] u(p)
\end{aligned}
$$

where $\mathrm{M}$ is the mass of the proton.

Let us first compare the different terms for helicity states, with spin along $z$. We will show one or two calculations explicitly for the second term from each decomposition, and the rest are given in Table 1. Writing $\bar{P}=p+p^{\prime}$ and working in the Chiral representation,

1. Helicity States (spin and momentum along z), $u^{\dagger}(p)=\left(\sqrt{E-p^{3}}, 0, \sqrt{E+p^{3}}, 0\right)$.

a) $\mu=0, \nu=1$, comparing second terms,

Ji (second term):

$$
\begin{aligned}
& \bar{u}\left(p^{\prime}\right) i B_{q, g}\left(\Delta^{2}\right)\left(\left(p+p^{\prime}\right)^{\mu} \sigma^{\nu \alpha}\right. \\
= & i \bar{u}\left(p^{\prime}\right) \gamma^{0}\left(\bar{P}^{0} \sigma^{10} \Delta_{0}+\bar{P}^{0} \sigma^{13} \Delta_{3}\right) u(p) \\
= & 0
\end{aligned}
$$

In going to the last line, we used the fact that in the Chiral representation, the surviving off-diagonal transverse-component $\sigma$ matrices will flip the spinor $u(p)$ in spin-space, making the spinor product $\bar{u}\left(p^{\prime}\right) u(p)=0$.

JM (second term) 


$$
\begin{aligned}
& A_{1}\left(\Delta^{2}\right) i\left(\varepsilon_{\mu \alpha \beta \sigma}\left(p+p^{\prime}\right)_{\nu}+\varepsilon_{\nu \alpha \beta \gamma}\left(p+p^{\prime}\right)_{\mu}\right) \Delta^{\alpha}\left(p+p^{\prime}\right)^{\beta} s^{\sigma} \\
= & \bar{P}^{0} \bar{P}^{3} \Delta^{0} s^{(2)} \\
= & 0
\end{aligned}
$$

So, they are identical for case a).

2. Canonical $x$-states, i.e. states with spin-projection along $x$-axis in rest frame but boosted to momentum along $z$-axis, $u_{x}^{\dagger}(p) \equiv \frac{1}{\sqrt{2}}\left(\sqrt{E-p^{3}}, \sqrt{E+p^{3}}, \sqrt{E+p^{3}}, \sqrt{E-p^{3}}\right)$

a) $\mu=0, \nu=1$, comparing second terms,

Ji (second term):

$$
\begin{aligned}
& \bar{u}\left(p^{\prime}\right) i B_{q, g}\left(\Delta^{2}\right)\left(\left(p+p^{\prime}\right)^{\mu} \sigma^{\nu \alpha}\right. \\
= & -\frac{i}{2} 2 \bar{P}^{0}\left(\Delta^{0}+\Delta^{3}\right) \sqrt{p^{0}-p^{\prime 3}} \sqrt{p^{0}-p^{3}} \\
= & -i \bar{P}^{0} \Delta^{+} \sqrt{p^{\prime-} p^{-}}
\end{aligned}
$$

JM (second term):

$$
\begin{aligned}
& A_{1}\left(\Delta^{2}\right) i\left(\varepsilon_{\mu \alpha \beta \sigma}\left(p+p^{\prime}\right)_{\nu}+\varepsilon_{\nu \alpha \beta \gamma}\left(p+p^{\prime}\right)_{\mu}\right) \Delta^{\alpha}\left(p+p^{\prime}\right)^{\beta} s^{\sigma} \\
= & 0
\end{aligned}
$$

following straightaway from the surviving components of the antisymmetric tensor for this state. 


\subsubsection{Further phenomenological development: Quark-Gluon Separa- tion}

Now, another interesting phenomenological thing to take note of is the following. In DVCS scattering, only quarks contribute directly to the total cross-section up to twist 2. It is a short-time, high-energy scattering process that according to the parton model lets us treat quarks as almost free and its remnants manifest as the entering of the gluon-field operator only in higher-twist objects. However, the angular momentum is precisely the second moment of the quark matrix-element entering DVCS (the one defining the GPDs) and is barely half of the total angular momentum contribution of, for example, the proton. This of course tells us that gluons were always kinematically there, just as spectators in electromagnetic scattering. It also serves well to remind ourselves that if one looked at a free hadron as a many-particle stationary state of fixed energy, then from first principles regarding entangled wave-functions, the only meaningful observables/eigenvalues are those of the total system, not of its individual degrees of freedom (particles). However, one can ask this question about separation of components phenomenologically in light of partonic interpretation at leading twist(s), forced gaugeinvariance etc; one may even "build" the hadron with free quarks and free gluons which then start interacting but retain some kinematical individuality. The hadronic angular momentum should be totally conserved where quarks and gluons share the contribution.

\subsubsection{Eigenstates of the Pauli-Lubanski Spin Vector}

While looking for tranvserse spin sum rules, one might want to know which states are appropirate ones to construct such a sum rule, and to relate to transversity correlators/GPDs etc. Some intuition may lead us to the Pauli-Lubanski spin vector, and to consider its eigenstates in the transverse direction. However, we will show below that the canonical spin state (boosted from rest-frame spinor to momentum $\vec{p}$ ) is in general not an eigenstate of the appropriate Pauli-Lubanski vector, $W_{\mu}=\varepsilon_{\mu \alpha \beta \gamma} J^{\alpha \beta} p^{\gamma}$, with $\mu$ being the direction of rest-frame spin projection of the spinor $u(p)$.

In fact, there are no eigenstates of this operator when we pick its component transverse to the momentum direction. We will show this presently. Again, working with momentum along $z$, let us find the eigenstates of the $x$-projection of the Pauli-Lubanski operator, $W_{1}$. 


$$
\begin{aligned}
W_{1} & =\varepsilon_{\mu \nu \rho \sigma} J^{\rho \sigma} p^{\nu} \\
& =\varepsilon_{1320} p^{3} J^{20}+\varepsilon_{1302} p^{3} J^{02} \\
& +\varepsilon_{1023} p^{0} J^{23}+\varepsilon_{1032} p^{0} J^{32} \\
& =-2\left(p^{3} J^{02}+p^{0} J^{23}\right) \\
& =\left[\begin{array}{cc}
\frac{1}{2}\left(p^{0} \sigma_{x}-i p^{3} \sigma_{y}\right) & 0 \\
0 & \frac{1}{2}\left(p^{0} \sigma_{x}+i p^{3} \sigma_{y}\right)
\end{array}\right] \\
& =\left[\begin{array}{cc}
W^{-} & 0 \\
0 & W^{+}
\end{array}\right]
\end{aligned}
$$

where $w^{+}$and $w^{-}$are defined to be the "Pauli-Lubanski ladder operators." In $4 \times 4$ matrix form,

$$
W^{1}=\left[\begin{array}{cccc}
0 & \frac{1}{2}\left(p^{0}-p^{3}\right) & 0 & 0 \\
\frac{1}{2}\left(p^{0}+p^{3}\right) & 0 & 0 & 0 \\
0 & 0 & 0 & \frac{1}{2}\left(p^{0}+p^{3}\right) \\
0 & 0 & \frac{1}{2}\left(p^{0}-p^{3}\right) & 0
\end{array}\right]
$$

Eigenvectors of the above operator are:

$\chi_{1,2,3,4}=\left(0,0, \frac{\sqrt{\left(p^{0}\right)^{2}-\left(p^{3}\right)^{2}}}{p^{3}-p^{0}}, 1\right)^{T},\left(\frac{\sqrt{\left(p^{0}\right)^{2}-\left(p^{3}\right)^{2}}}{p^{3}+p^{0}}, 1,0,0\right)^{T},\left(0,0,-\frac{\sqrt{\left(p^{0}\right)^{2}-\left(p^{3}\right)^{2}}}{p^{3}-p^{0}}, 1\right)^{T},\left(\frac{\sqrt{\left(p^{0}\right)^{2}-\left(p^{3}\right)^{2}}}{p^{3}+p^{0}}, 1,0,0\right)^{T}$.

This clearly does not solve the Dirac equation for a massive hadron, since it is either purely left handed or purely right-handed. Even in the limit of an infinite boost, the above eigenstates do not emerge for any spin projection. Thus, when a spinor is boosted transverse to its spin projection, we obtain a state which is not an eigenstate of transverse spin or any related transverse spin operator. This is important to note in the context of deriving sum rules and considering the effect of boosts on OAM/spin.

\subsubsection{Normalization of $T^{\mu \nu}$}

This is fine. It agrees dimensionally with usual Fock-space normalization. 


\subsubsection{Burkardt's Torque Analysis}

Mathias Burkardt [8] shows how two expectation values between proton states, one containing Ji's expression for OAM and the other JM's, differ by what seems to be a torque expectation value. He claims that this makes sense because if seen under the quarkdiquark model, there is a magnetic field responsible for a change in the struck quark's OAM as it travels through the rest of the 'proton'. However, this justification does not seem ample in light of a model-based approach for the proton's magnetic field affecting partonic OAM. Just because the two terms differ by what seems to be a torque term does not mean that this torque is the result of scattering, and that one is a pre-scattering quantity and the other post. The model is effective in describing many things, but to base the proton's bodily magnetic field and to consider a concept that is time-sensitive under this model may turn out to be a stretch. It is natural that the difference between two angular momenta will have units of torque. This is because a model is implicitly assumed.

\subsubsection{On a Comment from Leader's Paper}

Looking at Eq. (1.1),

$$
\left\langle p^{\prime} s\left|J_{i}\right| p s\right\rangle=2 p^{0}(2 \pi)^{3} \delta^{3}\left(\mathbf{p}^{\prime}-\mathbf{p}\right)\left(\frac{\mathbf{1}}{\mathbf{2}} \mathbf{s}_{\mathbf{i}}+\mathbf{i}(\mathbf{p} \times \nabla)_{\mathbf{i}}\right),
$$

one sees that there are two contributions in free Dirac theory to the nucleon's total spin: an orbital part and a spin part. A boost, being homogeneous in space when expressed by the EMT density, should not affect the orbital part. The spin, however, should be affected by a boost since it's sign must change if, for example, we reverse the sign of the boost (the projection direction for a start is at a changing angle to the boosted axis). Hence Eq. (1.8) cannot be the result of the calculation, independent of the frame. Indeed, Hatta et al show [20] that Ji's C-bar term enters the transverse spin sum rule, and is not independent of the longitudinal momentum. This means that the expectation value of transverse angular momentum for a hadron is not frame-independent but depends on the component of boost transverse to the $x$ or $y$ spin projection in the rest frame.

Also, there is a comment in [19] that the helicity state is different from the canonical state even if the boost is along the $z$-axis. However, the procedure is identical, with no 
scope for additional phases, so that part is not very clear. 


\section{Chapter 5}

\section{Vorticity}

\subsection{Introduction and Observations}

\subsubsection{The Nature of Vorticity}

This is a short chapter on some preliminary groundwork that has been done with regard to a potentially relevant physical quantity vis-a-vis angular momentum, the vorticus motion of the field or field vorticity. Classically, vorticity is defined as $\vec{W}=\nabla \times \vec{v}$ for any fluid. Starting with this definition, let us list some of our observations on vorticity, most of which may have been presented in other works.

1) Vorticity is a kinematical property of the fluid. It can be thought of as the ability to rotate objects placed in the fluid, rather than the rotation of the fluid by itself. If one places a paddle-wheel cylinder in a fluid with vorticity, it will tend to rotate, regardless of placement or orientation. This rotation will be caused by the necessary torque differential at its two edges.

2) Let us imagine placing a very thin cylindrical object in the plane of the fluid motion. Then, it is easy to see that if the fluid possesses vorticity transverse to the plane of motion, only then will the cylinder rotate where it is placed. And if it is a uniform cylinder, then it must rotate. So, it is a necessary and a sufficient condition to cause rotation.

If we start looking at macroscopic cylinders, then we see that if there is no vorticity in the fluid, there will be no torque differential at the edges to cause the object to rotate. However, if there is vorticity, then to see whether it will certainly cause rotation, we 


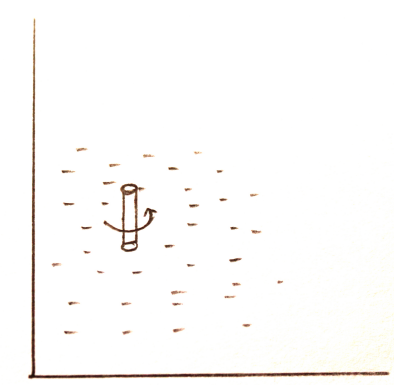

Figure 5.1: Cylinder rotates in fluid with vorticity

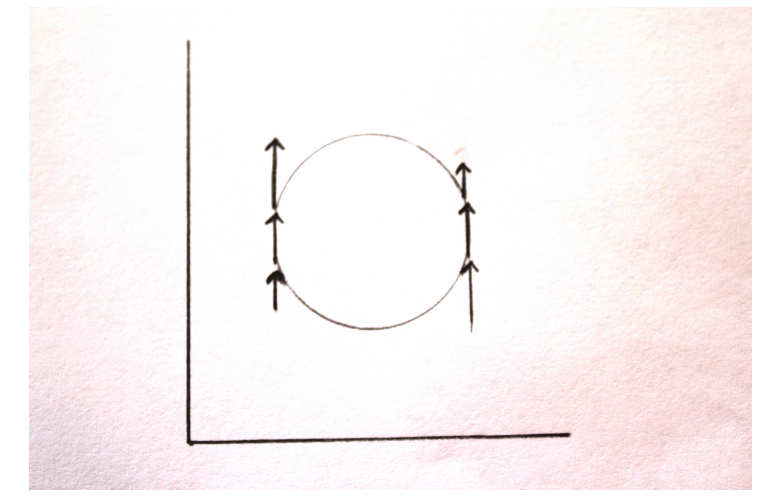

Figure 5.2: "Average" vorticity is zero: no rotation of cylinder. Top View.

have to consider an appropriately averaged value. For example, if the torque on the sides of a cylinder has a certain differential below the central horizontal line (top view) but that same differential exists with opposite sign at top, then the cylinder will not rotate.

3) One can understand the motion of an object in a fluid possessing vorticity as a rotation piece (curls) a likely translation piece (fluid pushes into the object). Curl does not measure 'pure rotation' but the existence of such a tendency. It can be seen as the inherent, "extrinsic" ability of the fluid to curl, and is somewhat like spin if seen as an intrinsic property of the field (though not confined anymore to a point in this interpretation).

For example, counterintuitively, a fluid whose velocity is defined by $v(r)=r w \vec{\theta}$, does cause a placed object to translate/revolve in addition to causing it to rotate, even though it is divergenceless and only has a curl. Divergence thus may imply a steady translation of the object in simple cases, but the absence of divergence does not guarantee pure rotation at a fixed spot. Regardless, a fluid can possess both vorticity and divergence. 


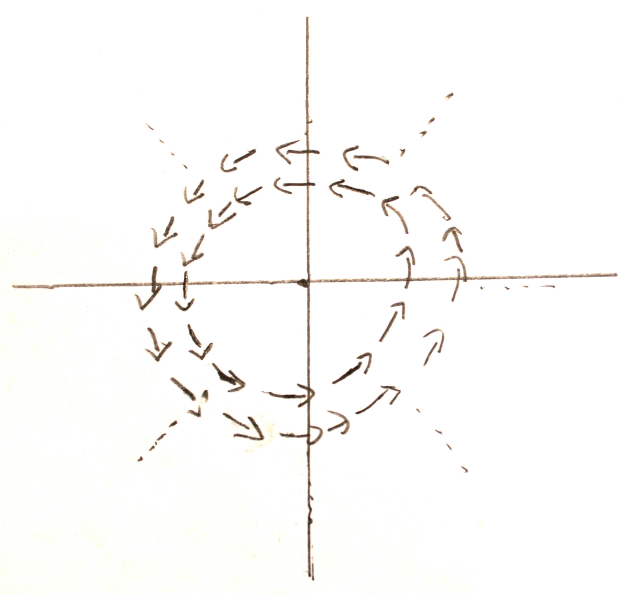

FiguRE 5.3: Uniform field does not have vorticity

Further, a circular symmetric field does not possess vorticity as long as it is uniform.

4) There is deviation from angular momentum properties: A classical field possessing constant angular momentum does not cause a thin uniform cylinder to always rotate (e.g. place it at the origin, and there is no vorticity or rotation here).

5) Force and velocity share a linear relationship. Let us orient our plane in the $\hat{z}$ direction. Zero curl of velocity implies that the increase in $y$-velocity in the $x$ direction is balanced by a corresponding increase in the $x$-velocity in the $y$ direction at all points. If a vector increase in velocity of the fluid is commensurate with the vector increase in force, regardless of the actual velocity, the placed object will then not undergo rotation. Thus the exerted force is, classically, linear with the velocity of the object in fluids where zero curl implies zero rotation.

Another way to think of the above situation is to pick any point and see which direction the fluid is flowing in at that point. One can fix this direction as one's base direction, and then traverse a step forward or to the right, to see that the condition of zero curl in the direction perpendicular to the plane in question [imagine planar disc of fluid with points A, B,C,D] results in the fluid closing in on itself. This can be thought of as the fluid curling into itself and thus cancelling out its potential for curl. The measure for vorticity or curl of a field is such that an increase in its $x$ component in the $y$ direction is considered equal and opposite to an increase of the $y$ component in the $x$ direction. 
6) Vorticity integrates to zero over any closed surface: $\iint_{S} \vec{W} \cdot d a=\oint_{C} \vec{v} \cdot d l=0$ if we take a closed surface, which leads to an infinitesimally small loop to integrate over via Stokes' theorem. The latter quantity, for open surfaces, defines the circulation.

7) For fluids, the natural variable is not particle angular momentum, but its curl and divergence at various points. If one sees a classical field as possessing properties of a fluid - existing dynamically at every point and pervading all of space- one can understand the merit of studying its vorticity.

\subsubsection{Extension to Field Theory: Vorticity Density}

We define vorticity to be the curl of the momentum density, the energy momentum tensor: $\nabla \times T^{o i}(x)$. Curl of small volume $\Delta V$ packets of momentum $\Delta V T^{0 i}(x)$ that are adjacent are independent of size of packet for small packets and reduce, essentially, to the definition of vorticity density. Hence, one can think of the definition $\nabla \times T^{o i}(x)$ to be reflecting the curl of packets of momentum in a sense. Instead of velocity field now we have momentum density field, physical meaning given above. From a classical point of view, this makes sense since both velocity and mass density of fluid responsible for causing rotation. When the expectation value is taken, the curl moves out of the matrix element and thus the curl of the expectation value of momentum density is taken at a particular point. Vorticity is not a manifestly covariant quantity because it is a curl, just the way angular momentum is not covariant. However, it is a sensible framedependent observable that has physical meaning in the frame in which it is being viewed.

\subsubsection{The Circulation}

Let us calculate the circulation of the EMT around a loop, $\oint_{C} \overrightarrow{T^{0 i}}(x) \cdot d l$, which can of course be expressed also as the surface integral of its vorticity, i.e. $\iint\left(\nabla \times T^{0 i}(x)\right) \cdot d a$. Let us focus on the perpendicular components of momentum density by choosing a hemispherical surface to be oriented in the $z$ direction so that its "mouth" lies in the $x-y$ plane. One can imagine for visualization the curl being integrated over its surface so that the circulation is calculated around its rim, having radius $r_{0}$. One may take the limit of a very large surface/hemisphere at the end. 


$$
\begin{aligned}
& \iint \phi^{*}\left(p^{\prime}\right) \phi(p) d^{3} p^{\prime} d^{3} p \oint_{C}\left\langle p^{\prime}\left|\overrightarrow{T^{0 i}}(x)\right| p\right\rangle \cdot d l \\
= & \iint e^{i \Delta \cdot x} \phi^{*}\left(p^{\prime}\right) \phi(p) d^{3} p^{\prime} d^{3} p r_{0} d \theta\left(-\left\langle p^{\prime}\left|\overrightarrow{T^{0} x}\left(r_{0}, \theta\right)\right| p\right\rangle \sin (\theta)+\left\langle p^{\prime}\left|\overrightarrow{T^{0} y}\left(r_{0}, \theta\right)\right| p\right\rangle \cos (\theta)\right) \\
= & \iiint e^{i \Delta_{x} r_{0} \cos (\theta)+i \Delta_{y} r_{0} \sin (\theta)} \phi^{*}\left(p^{\prime}\right) \phi(p) d^{3} p^{\prime} d^{3} p r_{0} d \theta\left(-\left\langle p^{\prime}\left|T^{0 x}\left(r_{0}, \theta\right)\right| p\right\rangle \sin (\theta)\right. \\
+ & \left.\left\langle p^{\prime}\left|T^{0} y\left(r_{0}, \theta\right)\right| p\right\rangle \cos (\theta)\right) \\
= & \iint \phi^{*}\left(p^{\prime}\right) \phi(p) d^{3} p^{\prime} d^{3} p\left(-A_{s}\left(p^{\prime}, p, r_{0}\right)\left\langle p^{\prime}\left|\overrightarrow{T^{0} x}\left(r_{0}, \theta\right)\right| p\right\rangle\right. \\
+ & \left.A_{c}\left(p^{\prime}, p, r_{0}\right)\left\langle p^{\prime}\left|T^{0} y\left(r_{0}, \theta\right)\right| p\right\rangle\right)
\end{aligned}
$$

where $A_{s}\left(p^{\prime}, p, r_{0}\right)=\int e^{i \Delta_{x} r_{0} \cos (\theta)+i \Delta_{y} r_{0} \sin (\theta)} r_{0} d \theta$ and $A_{c}\left(p^{\prime}, p, r_{0}\right)=\int e^{i \Delta_{x} r_{0} \cos (\theta)+i \Delta_{y} r_{0} \cos (\theta)} r_{0} d \theta$. We have stripped the $r$-dependence of the matrix element, which now only depends on the center of the hemisphere! This quantity, the circulation, is clearly gauge invariant. In the limit of a very large hemisphere, it will disappear only if the expectation value of momentum density disappears at all points far away from the center.

For the same reason, when we consider the circulation around an infinite plane, we get zero identically. Orienting the plane in the $x-y$ plane,

$$
\begin{aligned}
\oint_{C} \overrightarrow{T^{0 i}}(x) \cdot d l & =\iint\left(\nabla \times \overrightarrow{T^{0 i}}(x)\right) \cdot d a \\
& =\iint\left(\nabla \times T^{0 i}(x)\right)^{z} d x d y \\
& =\iint\left(\partial_{x} T^{0 y}-\partial_{y} T^{0 x}\right) d x d y
\end{aligned}
$$

The last line disappears if the momentum "density" is zero far away from the "localized" particle or state, where its scope can be thought to disappear. Some authors may pedantically question this in the quantum theory, but in the above viewing of things it is quite clear why the expectation value at large distances must disappear. 


\subsubsection{Vorticity is a Conserved Charge}

Vorticity, as defined above, is a conserved charge. This can be seen starting from conservation of the current associated with four-momentum:

$$
\begin{aligned}
& \partial_{\mu} T^{\mu \nu}(x)=0 \\
& \partial_{t} T^{0 \nu}=-\partial_{i} T^{i \nu}
\end{aligned}
$$

Taking the curl of both sides, and focusing on $\nu=k=1,2,3$,

$$
\begin{aligned}
\Longrightarrow \varepsilon^{i j k} \partial_{j} \partial_{t}\left(T^{0 k}\right) & =-\varepsilon^{i j k} \partial_{j} \partial_{l} T^{l k} \\
\partial_{t} \varepsilon^{i j k} \partial_{j} T^{0 k} & =-\partial_{l} \varepsilon^{i j k} \partial_{j} T^{l k}
\end{aligned}
$$

These are 3 equations, one for each value of the index $i=1,2,3$. Each of these equations is a conserved current law. This current is the vortex current, and there is a conserved charge associated with each $i$,

$$
V^{i}=\int d^{3} x \varepsilon^{i j k} \partial_{j} T^{0 k}(x)
$$

It is clear that $\partial_{t} V^{i}=0$. In standard 3-vector notation, the conservation equations become

$$
\partial_{t}\left(\nabla \times T^{0 k}(x)\right)=-\nabla_{l} \cdot\left(\nabla_{k} \times T^{l k}(x)\right)
$$

and the conserved charge becomes $V^{i}=\int d^{3} x \nabla_{k} \times T^{0 k}(x)$. It is understood that the above equality refers to 3 equations. 
We now quote some general observations:

1) Vorticity is a conserved charge.

2) This charge, when its density is integrated over all space, turns out to be zero since the density is a curl.

3) It (the expectation value) is gauge-invariant.

4) It is a conserved charge, yet its definition is not manifestly covariant. This is not to say that it does not hold in other frames of course, just that it does not do so via a standard Lorentz transformation.

5) The vorticity density itself will be constant in time (no flow of charge in space) if the "shear vorticity," $\nabla_{k} \times T^{l k}(x)$, is symmetric in its two indices. This is because it will be the curl of the divergence of the same vector now.

\subsection{Calculations: Relating Vorticity and Angular Momen- tum}

\subsubsection{Involving Off-diagonal Elements of EMT}

Symmetry of curl of shear terms implies constant vorticity. The flux surfaces already represent a kind of curling in space when one goes from the $i=1$ surface to the $i=2$ and then the $i=3$ surface. This vector quantity is further curled. And then we question the symmetry of the two surviving indices, one of which is the new curled index and the other one represents originally the component of momentum that is added across the flux surface. A simple condition of constant charge density implies something quite non-trivial with the shear terms thus.

Aside: By the way, when writing these surfaces it is assumed that all the momentum flux is coming from below this surface, for each surface, despite concerns of "over-counting".

\subsubsection{Writing Momentum as a Function of Vorticity}

One may think of vorticity as an independent observable, but it helps to see how it relates to angular momentum. Using a vector calculus identity, 
$\vec{T}^{0 i}=-\nabla \frac{1}{4 \Pi} \int \frac{\nabla \cdot \vec{T}^{0 i}\left(x^{\prime}\right)}{\left|x-x^{\prime}\right|} d^{3} x^{\prime}+\nabla \times \frac{1}{4 \Pi} \int \frac{\vec{W}\left(x^{\prime}\right)}{\left|x-x^{\prime}\right|}$

In particular, if the EMT is divergenceless (which also means that the energy density of the state is fixed in time), we obtain

$\vec{T}^{0 i}=\nabla \times \frac{1}{4 \Pi} \int \frac{\vec{W}\left(x^{\prime}\right)}{\left|x-x^{\prime}\right|}$,

an expression for the momentum density just in terms of vorticity.

\subsubsection{Evaluating the Becattini Term: Redefinition of $\mathrm{J}$ in terms of vorticity}

We will see below that there is merit to defining orbital angular momentum as $\left\langle\int d^{3} x \frac{x^{2}}{2} \nabla \times\right.$ $\left.\overrightarrow{T_{C}^{0 i}}(x)\right\rangle$. When the simple replacement $T_{C} \rightarrow T_{B}$ is made, we arrive at total angular momentum, $J$ ! This is not unlike using the Belinfante EMT to define $\vec{J}=\vec{r} \nabla \vec{T}^{i}{ }_{B}$ instead of the canonical EMT, which would not yield a spin term. It would yield, it is of note, Ji's orbital contribution rather than Jaffe-Manohar's. So, is it inherently more natural to define orbital angular momentum using the vorticity definition as above? What makes it more natural? The physical spinning/vorticus form, due to the explicit cross derivative on the expectation value of the density, rather than intrinsic form? It was more difficult in principle to disentangle quark from gluons in their AM contribution, but separating explicit orbital from the more intrinsic/subtle spin seems natural.

Defining $T^{k}\left(p^{\prime}, p\right) \equiv\left\langle p^{\prime}\left|\Pi^{k}(0)\right| p\right\rangle, \Delta \equiv p-p^{\prime}$ and $A^{i j \alpha \beta} \equiv T^{k} \epsilon^{i j k} \delta^{\alpha} \delta^{\beta}$ 


$$
\begin{aligned}
& \left\langle\int d^{3} x \frac{x^{2}}{2} \nabla \times \vec{\Pi}(x)\right\rangle^{i}=\frac{1}{2} \lim _{k \rightarrow 0} \int d^{3} x d^{3} p d^{3} p^{\prime} \phi(p) \phi\left(p^{\prime}\right) e^{i k x}\left\langle p^{\prime}\left|x^{2} \partial^{j} \Pi^{k}(x)\right| p\right\rangle \epsilon^{i j k} \\
& =\frac{1}{2} \lim _{k \rightarrow 0} \int d^{3} x d^{3} p d^{3} p^{\prime} \phi(p) \phi\left(p^{\prime}\right) e^{i(k+\Delta) \cdot x} \Delta^{j} x^{2}\left\langle p^{\prime}\left|\Pi^{k}(0)\right| p\right\rangle \epsilon^{i j k} \\
& =\frac{1}{2} \lim _{k \rightarrow 0} \int d^{3} p d^{3} p^{\prime} \phi(p) \phi\left(p^{\prime}\right) e^{i(k+\Delta) \cdot x} d^{3} x \Delta^{j} x^{\alpha} x^{\beta} T^{k}\left(p^{\prime}, p\right) \epsilon^{i j k} \delta^{\alpha} \delta^{\beta} \\
& =\frac{1}{2} \lim _{k \rightarrow 0} \int d^{3} p d^{3} p^{\prime} \phi(p) \phi\left(p^{\prime}\right) \frac{\partial}{\partial k^{\alpha}} \frac{\partial}{\partial k^{\beta}} e^{i(k+\Delta) \cdot x} d^{3} x \Delta^{j} T^{k}\left(p^{\prime}, p\right) \epsilon^{i j k} \delta^{\alpha} \delta^{\beta} \\
& =\frac{1}{2} \lim _{k \rightarrow 0} \int d^{3} p d^{3} p^{\prime} \phi(p) \phi\left(p^{\prime}\right) \frac{\partial}{\partial k^{\alpha}} \frac{\partial}{\partial k^{\beta}} e^{i(k+\Delta) \cdot x} d^{3} x \Delta^{j} A^{i j \alpha \beta}\left(p^{\prime}, p\right) \\
& =\frac{1}{2} \lim _{k \rightarrow 0} \int d^{3} p d^{3} p^{\prime} \phi(p) \phi\left(p^{\prime}\right) \frac{\partial}{\partial k^{\alpha}} \frac{\partial}{\partial k^{\beta}} \delta^{(3)}(k+\Delta) \Delta^{j} A^{i j \alpha \beta}\left(p^{\prime}, p\right) \\
& =-\frac{1}{2} \lim _{k \rightarrow 0} \frac{\partial}{\partial k^{\alpha}} \frac{\partial}{\partial k^{\beta}} \int d^{3} p \phi(p) \phi^{*}(p+k) k^{j} A^{i j \alpha \beta}(p+k, p) \\
& =-\frac{1}{2} \lim _{k \rightarrow 0} \frac{\partial}{\partial k^{\alpha}} \frac{\partial}{\partial k^{\beta}} \int d^{3} p \phi(p) \phi^{*}(p+k) k^{j} A^{i j \alpha \beta}(p+k, p) \\
& =-\frac{1}{2} \lim _{k \rightarrow 0} \epsilon^{i j k} \delta_{\beta}^{\alpha}\left[\delta _ { j \alpha } \left(\int d^{3} p \phi(p)\left\langle p\left|\Pi^{k}(0)\right| p\right\rangle \frac{\partial}{\partial k_{\beta}} \phi^{*}(p+k)\right.\right. \\
& \left.+\int d^{3} p|\phi(p)|^{2} \frac{\partial}{\partial k_{\beta}}\left\langle p+k\left|\Pi^{k}(0)\right| p\right\rangle\right) \\
& +\delta_{j}^{\beta}\left(\int d^{3} p \phi(p)\left\langle p\left|\Pi^{k}(0)\right| p\right\rangle \frac{\partial}{\partial k_{\alpha}} \phi^{*}(p+k)\right. \\
& \left.\left.+\int d^{3} p|\phi(p)|^{2} \frac{\partial}{\partial k_{\alpha}}\left\langle p+k\left|\Pi^{k}(0)\right| p\right\rangle\right)\right] \\
& =-\lim _{k \rightarrow 0} \epsilon^{i j k}\left(\int d^{3} p \phi(p)\left\langle p\left|\Pi^{k}(0)\right| p\right\rangle \frac{\partial}{\partial k_{j}} \phi^{*}(p+k)\right. \\
& \left.+\int d^{3} p|\phi(p)|^{2} \frac{\partial}{\partial k_{j}}\left\langle p+k\left|\Pi^{k}(0)\right| p\right\rangle\right)
\end{aligned}
$$

This is to be exactly compared to (as in the wave packet relation of AM and the EMT),

$$
\begin{aligned}
<J^{\mu \lambda}> & =\lim _{\mathbf{k} \rightarrow \mathbf{0}}(-\mathbf{i}) \int \mathbf{d}^{3} \mathbf{p} \phi(\mathbf{p})\left\langle\mathbf{p}\left|\mathbf{T}_{\mathbf{B}}^{\mathbf{0 \lambda}}(\mathbf{0})\right| \mathbf{p}\right\rangle \frac{\partial}{\partial \mathbf{k}_{\nu}} \phi^{*}(\mathbf{p}+\mathbf{k})-(\lambda \leftrightarrow \nu) \\
& +(-\mathbf{i}) \int \mathbf{d}^{3} \mathbf{p}|\phi(\mathbf{p})|^{2} \frac{\partial}{\partial \mathbf{k}_{\nu}}\left\langle\mathbf{p}+\mathbf{k}\left|\mathbf{T}_{\mathbf{B}}^{\mathbf{0}}(\mathbf{0})\right| \mathbf{p}\right\rangle-(\lambda \leftrightarrow \nu)
\end{aligned}
$$

It is understood above that $J^{12}$ means $J^{3}$ etc.

If, in particular, we replace $\overrightarrow{\Pi^{k}}$ by $-T_{B}^{0 k}$, we will get 


$$
\begin{aligned}
\left\langle\int d^{3} x \frac{-x^{2}}{2} \nabla \times T_{B e l}^{0 k}(x)\right\rangle^{i} & =\frac{1}{2}\left\langle J^{i}\right\rangle+\frac{1}{2}\left\langle J^{i}\right\rangle \\
& =\left\langle J^{i}\right\rangle
\end{aligned}
$$

Q.E.D.

Aside:

Note that the derivative was taken outside the bra-ket in the first line. This is not done usually (e.g. in the evaluation of $T^{\mu \nu}$ ) but here it is natural to do so. There are two reasons for this. In field theory, the coordinate space is in a sense disconnected from the Hilbert space (Fock space) and an operator which has an explicit spatial derivative classically does not have much meaning if the derivative is taken inside the expectation value, just acting on the spatial coefficient of the ket. Thus, it applies to the expectation value of the operator, which is spatially dependent. This is similar to orbital angular momentum in the free theory being $\vec{r} \times T^{0 i}$, where the expectation value of momentum is the quantity of interest that then takes the spatial coordinate into account to define orbital angular momentum. Similarly, vorticity deals post priori with the momentum density, after its expectation value has been taken.

Also, imagine translating a state along some axis, say, to the left. If this is done, both the bra and the ket are translated (or the operator is equivalently translated from both sides) to give physical meaning to the evaluation (which is a physical expectation value). The physically relevant quantity in Hilbert space is the expectation value and the spatial derivative cannot only apply to the ket in an isolated fashion. 


\section{Chapter 6}

\section{Conclusions and Philosophy}

\subsection{Summary of Contributions}

A pointwise summary of some contributions made here will not compromise the nature of it. Following are some of the things that resulted from our work:

1) We constructed and presented our wave packet spin sum rule and commented on why it is a necessary starting point for spin sum rules. This gives us a general formula for evaluating any component of angular momentum in any generic state in terms of the gravitomagnetic form factors of the EMT. We pointed out a momentum-space density for the angular momentum operator in terms of wave packets. We discussed misconceptions in this aspect of the sum rule, like the momentum derivative should be with respect to the ket momentum, and not the difference of momenta $\Delta$, since the latter is ambiguous and will give a different result depending on where it is performed.

We also presented associated phenomena and showed that the proton behaves like an effective fermion under a shift of origin. In particular, we showed translation invariance of the spin (magnetic dipole moment) of an effective hadronic spin state, such as a hadron at rest or one possessing azimuthal symmetry in its wave function. We showed that azimuthal symmetry should be considered in momentum space so that the notion of azimuthal symmetry in the context of OAM can be extended to composite or multiparticle states. We also motivated why the term "spin crisis" makes sense in this context using a classical, quantum mechanical and field theoretical example respectively to appreciate the same physical observable. The term also would arise in the context of the parton model, as one did not expect real orbital angular momentum to contribute. One may 
have posited that there would be no longitudinal OAM coming from the parton model due to limited transverse momentum and spin, as measured in PDFs, was expected to carry all the angular momentum (even though the required difference was a very small number in the context of quantized angular momentum of the first excited state, $\left.\frac{h}{4 \Pi}\right)$.

2) We presented a GPD spin sum rule for spin-1 systems $\left(\frac{1}{2} \int d x x H_{2}(x, t, \zeta)=1\right)$ and commented on the partonic interpretation. In analogy with the spin $\frac{1}{2}$ case, this GPD represents the magnetic form factor (via first moment), $G_{M}$ of the deuteron. We compared orbital angular momentum to the case of the proton and showed that due to isoscalarity, the contribution of the up and down quarks is very small in the orbital angular momentum of the deuteron. In the process, an operator product expansion was also done on the energy momentum tensor for spin 1 hadrons, using polarization vectors that arise from effective Proca theory for spin 1 particles. Finally, we constructed observables that would help measure the GPD, $\mathrm{H}_{2}$, as part of the next step.

3) We constructed a formalism for the helicity amplitudes of spin-1 hadrons (using GPDs) that separates spin dependence from momentum dependence in the amplitudes. We also presented a general form not seen before for the spin-1 polarization vectors, all the while using Lorentz Transformations to write quantities as a function of rest-frame spinors. This was developed to be on equal footing with the nucleon case.

4) We point out that X.Ji et al's Pauli-Lubanski vector treatment [15] of angular momentum is helped by the realization that $W^{\perp}=J^{T}$ ! This is not just true in the rest frame, but in a longitudinal momentum eigenstate or any state with an azimuthally symmetric wave packet. So, their results really apply directly to transverse angular momentum and can be compared directly to the longitudinal case, both operators being in their original canonical forms. We emphasize that since $\vec{J}\left(M^{\mu \nu \lambda}\right)$ is not covariant, one cannot simply replace the "0" component with the "+" component, or harbor such notions a priori, with operators like $J^{01}=M^{001}$. So, we must look at the way the operators originally stand before immediately extending them to the light cone forms, since they arise from a cross product.

5) a) We recalculated $\left\langle J^{z}\right\rangle$ and $\left\langle J^{\perp}\right\rangle$ for the proton in a helicity state and showed that the former is zero up to leading twist. We compared these results to the deuteron case and found the similarity that although the longitudinal angular momentum is not zero up to twist 2 , it is still relatively suppressed by $P^{+}$compared to transverse angular momentum. We gave physical/phenomenological arguments why this is the case and 
discussed that there may be lack of azimuthal symmetry leading to a degeneracy in proton states. We also discussed the twists of these different operators and how the order at which $P^{+}$appears should be our criterion for twist/suppression, as we are dealing with the operator product of operators having different spin indices (correlator current, angular momentum etc).

b) We discussed how boosts affect small classical current loops/magnetic dipoles to understand how they might affect spin in field theory. We saw that parallel boosts do not affect spin projection, and boosts transverse to spin projection shrink it to zero, in agreement with the results we found for a point-like particle, whose structure does not contain the off-forward GPD $E(x, t, \zeta)$. We saw frame-dependence in the case of the latter boost, same as with hadrons.

6) We also showed that the magnetic form factor $G_{M}$ directly enters the longitudinal case of the deuteron but in the transverse case there are other contributions that were earlier aloof, which is interesting. Also, the skewness function, $b_{1}=H_{5}(x, 0,0)$ does not enter the longitudinal or transverse angular momentum in the deuteron. This was surprising and it was discussed how this implies a delicate cancellation of partonic angular momentum contributions coming from skewness.

7) Using azimuthal symmetry, rotation invariance and boosts starting from the rest frame, we showed that that boosts and rotations commute about the same axis not just for 4 -vectors, but also for spinors and thus as long as angular momentum is measured in the direction of the boost, the boost does not affect it for any state. For example, $\left\langle J^{z}\right\rangle$ does not change even in a state initially transversely polarized at rest and then boosted to some longitudinal momentum. If the above expectation value is taken in a longitudinally polarized state, it is of course unchanged. This is as expected and is in line with the notion that a boost should not affect angular momentum parallel to its direction, since momentum will only change in that direction, leaving $\vec{r} \times p$ unchanged.

Transverse angular momentum is zero in the longitudinally polarized case due to azimuthal symmetry, before and after a longitudinal boost. However, $\left\langle J^{\perp}\right\rangle$ for a transversely polarized state does change after a boost is performed, even though the longitudinal does not! This is different from the non-relativistic QM formalism, where a general spin state is uniquely given in a basis along any axis of projection. In the case of spin $\frac{1}{2}$, we see especially that this cannot be accommodated without degeneracy of longitudinally polarized states. The new state is a sum over different eigenstates of longitudinal angular momentum, and provides a different transverse expectation value, since a boost 
changes the actual shape of the wave function. Specifically, it changes the azimuthal probability density, not the longitudinal and hence longitudinal angular momentum is unaffected.

8) We reviewed the operators describing hadronic angular momentum and hopefully made some useful comments and clarifications.

9) We discussed a potentially new relevant observable, field vorticity, $W=\nabla \times T^{\mu \nu}(x)$ and discussed a new conservation law in terms of shear terms of the EMT. We related angular momentum to vorticity thus: $\vec{J}=-x^{2} \nabla \times \vec{T}_{B}^{0 i}(x)$. It is likely a twist-3 observable.

10) A perspective on gauge-invariance and operator separability was presented. Since the QM formalism designates observable operators for each particle in a multiparticle state separately without entanglement at the operator level, we are motivated to look for a similar division in field theory. However, a clear partonic interpretation of this division is possible up to leading twist only and thus we should not insist that the angular momentum operators, which are blind to twist expansion, be gauge-invariant individually. These various quantities in any decomposition done correctly may well represent what they claim to, but are also inherently tied with the rest, like water and wetness. Various other perspectives on this and related issue were given throughout the document.

11) Numerous other small points and pedagogical observations were made, such as explaining the fact that off-forward momentum matrix elements should conserve currents so that these are generally conserved in a physical wave packet state etc.

\subsection{Brief Points of Philosophy}

Many patterns are revealed in nature through our observations and experience. Sometimes these are like fractals. When we explore one aspect closely, we see a microcosm of the same essential universal laws, revealed again upon taking a narrower point of view. Sometimes, we see apparently new laws. For example, when we go from classical physics to quantum mechanics or field theory, we go from more manifest views to more subtle views. Angular momentum can be seen classically in a rotating object, but in field theory it seems to be a more abstract/subtle quantity. It is still very much physical 
and the notion is still of angular momentum at the essence of it, only that it is seen and measured differently. Spin, in particular, is so subtle that it does not carry an explicit spatial character, even though its nature is that of a rotating particle! Thus, at the heart of it, these operators in the different theories represent the same thing, but in one case explicitly and in another more implicitly. The fact that they arise from and are agents of the same symmetry of nature (e.g. angular momentum are conserved quantities linked to rotation of space in classical mechanics and classical field theory and are generators of rotation as well in the quantized theory) is a symptom of the above.

A more explicit example of seeing different physical mechanisms at work in nature is the following. At low energies, the proton responds to probes like an effective single fermion. Deep Inelastic Scattering experiments show the proton to be a semi-classical assembly of "point-like" quarks in momentum space and parton densities obtain in its description. If we view it at a deeper level, we see GPDs entering the description at the amplitude-sum level, like a path integral, where we sum over all subtle structure functions that determine the underlying character of the proton. However, we don't completely "dispense" with the parton model. There is still some lingering relative truth/merit to it. This is either because it is still a valid deeper aspect of how the hadron is structured, or we have not gone deep enough in our subtle understanding of nature yet. So, these different commentaries on physical phenomena can complete with each other in our understanding, and therefore there is some relative nature to them. If our understanding was more complete, things would probably end up being less relative, and the case of priority given to one physical aspect over another in describing a phenomenon, depending on the thinker in question, may give way. There is surely a complete underlying pattern that governs these theories, since they are not independent of each other and therefore can be harmonized by the underlying principle. What the philosophical nature of that underlying principle is, we shall not attempt a discussion in that direction here. Also, the same model description for something can be a very good description of one aspect (e.g. scattering statistics) but not of another physical aspect (e.g. angular momentum/spin). The model can thus incorporate more relevantly features of scattering, but if it were a quite complete physical description, or at least a very refined model, it would cover all aspects equally well.

It may also be said here that a good scientist/natural philosopher can exercise different points of view when viewing the same physical situation, only with different lenses/depths. Imagine a classical charge distribution on a conductor, thrown together at some initial time. These charges will move away very fast, gaining momentum. As far as we can see, the momentum is carried by the charges and there are no photons involved. 
This is the classical view of the situation. The same situation, when viewed with a quantum (subtler) vision reveals that if the time interval of our viewing was very short, then we would "see" the virtual photons being exchanged. At any given point, it would be hard to say whether the momentum is carried by the electrons or these virtual photons separately, as one cannot pinpoint a start time and an end time for their absorption and emission in this entangled state. They are exchanged at a less physically obvious level, at a deeper/more subtle level we can say. There is an uncertainty, however small, during which this exchange occurs. This is a good example of a classical situation one can appreciate with quantum lens. One cannot talk about the electrons or the photons disjointly in quantum field theory in an interacting state - one needs to consider the state as a whole. Similarly, when talking about angular momentum content, one can separate the plausible contributions by various criteria outlined before, but at the same time one must consider that the quantities are mutually inclusive in a deeper sense.

Further, it is not the case that classical laws of physics have less merit than their quantum counterparts. Their clean elegance has truth and merit, just that they do not obtain in certain situations physically. It is not that they are inherently, at the heart of their formulation, plagued with the uncertainties corrected by/arising from quantum descriptions. Measured physical behavior of particles, though, seems to suggest this in a direct practical sense. In the author's point of view, it is about the way the classical laws are seen and understood that decides the depth of description rather than the classical physical observations in and of themselves.

One can also appreciate with depth of probing in the case of a proton that some aspects of our superficial viewing of it were unsatisfactory. The quarks in the immediate view suggested by the naive quark model make up the proton effectively, but do not describe it completely. When our "gloss" from our proton view is removed slowly, we go from seeing it as a semi-classical PDF assembly (to 90 per cent accuracy in experiment) to a more elegant second order partonic description that involves summing over the different quark fields at the amplitude level to describe the overall scattering amplitude of the proton. This is where GPDs enter, as explained in Chapter 1. When the gloss is further removed, gluon fields enter the description, and they can exchange at the perturbative or non-perturbative level with quarks from the above descriptions. So, going deeper, it gets much more complicated, when one takes into account the gluons and quarks softly interacting with each other and yet interacting with the probe in a particular way. As the gloss is removed, what one assumed to be abstracted in the higher-level objects, like PDFs, in an incomplete way, comes to the fore. However, our perspective in these treatments is very important because one may sacrifice the bigger picture getting carried 
away and placing too much emphasis on isolated aspects of the structure. The removal of the "gloss" does not mean that we see the initial descriptions as totally wrong or not physically occuring, but they surrender to/are subsumed in a more elegant and deeper point of view.

\subsubsection{Research Perspective}

The current perspective in work in the field of physics is, from the author's point of view, a bit removed from the essence of physics. Physicists, theoretical and experimental, tend to at times complicate things far more than necessary in the name of rigor. If our goal is to eat, we do not get lost in the cutlery. A more fundamental physical argument may be staring us in the face, but we may be physically conditioned to do things a certain unnatural way. Part of the goal of this work is to show that simple treatments involving, boosts, symmetries, different frames of reference, wave packets, vorticus etc. can in their own nice way give deeper insight into hadron structure. In this document, efforts were made to present some new perspectives on things we already know.

Further, it cannot be the best way to go if one begins to lose sight of the very reason they were attracted to the field of physics. Sure, there are practicalities involved in all kinds of endeavor, but when one loses oneself in isolated aspects of large-number-loop calculations, one begins to lose touch with the essence and reason of why they started off in the field. Mathematics in physics (and in a "purer" sense) can be very elegant, but that is not the kind of issue being discussed here.

Finally, as a last point of view, one should not be afraid to spend more time on the natural philosophy behind one's physical results. This is done, but perhaps not as extensively as merited by the field. By themselves, the results are of no use unless one makes or implies a clear statement of underlying reason/philosophy stemming from it. Philosophy, or "the love of wisdom," is not to be considered alien if done properly and simply and is necessary to present as well as gain a deeper understanding of one's work. After all, it is not the physical result by itself that is most important: it is what drives it, what is at the heart of it. The essential conclusion. At times, there may be debatable or relative perspectives, or more than one aspect of nature can be simultaneously shown (in one sense this is always true) by one observation. 
The reader is thanked for his/her patience in reading this manuscript. 


\section{Appendix A}

\section{Review}

\section{Formalism}

One obtains the classical energy-momentum tensor by use of Noether's theorem. One then promotes this to a QFT operator and uses it between states to calculate observables like four-momentum. The operator implements translations on states in Fock space. Similarly, the classical angular momentum operator is obtained by infinitesimally rotating the frame of reference and identifying corresponding conserved quantities resulting from this invariance of the theory (Lagrangian) under such different rotations. These field-quantities are also then promoted to operators that rotate quantum states in Fock space. In these cases, in the literature, "canonical" is used synonymously with "physical" and is not to be confused with the canonical Hamiltonian prescrptiption. Let us now briefly remind ourselves that the Poincare group generators implement rotations and translations for classical fields in the Lagrangian (like the spin $\frac{1}{2}$ representation of the Lorentz group does for rotating/boosting the Dirac spinor) and states in Hilbert space (Fock space) are transformed by the quantized field operators like in ordinary quantum mechanics. The Dirac representation of the Lorentz group contains matrices that contain the $2 \times 2$ Pauli matrices are responsible for rotating the two-component "spinor" in the unquantized Dirac field.

\section{Why aren't we being careful with spin?}

A sensible sum-rule can still be obtained if one composes one's state of almost longitudinally polarized momentum states (with small transverse components, around a state peaked along a certain longitudinal momentum, to allow for the sum rule calculation). Some thought reveals that this expectation value (even with interfering phases) should be order $\epsilon$ different from $\frac{1}{2}$, the value of spin in a purely longitudinal state, where $\epsilon$ reflects 
the degree to which we are off-peak in momentum. Even though it is clear that changing the momentum in a ket by itself affects the helicity of the state, the expectation value of angular momentum in a superposition of kets that are peaked longitudinally does not change from the original longitudinal value. This superposition would be precisely the state that experimentalists deal with when they measure the spin of a 'longitudinal state'. Thus, the objection that momentum and spin are not independent in a ket does not affect our results in this case for a sum rule.

\section{Comment on EM tensor-decomposition objection}

There was an objection raised in literature regarding the off-forward decomposition of the matrix element of the EMT. We see this in [Stapp] and even though one may argue that the two forms (Ji and JM) change with identical Lorentz transformations, the JM form is not a priori permissible. The total tensor form, however, does transform covariantly as a Lorentz tensor if the Dirac indices are disregarded and the individual momenta etc. are not transformed. In other words it is sufficient to regard the entire matrix element as if it were a constant Lorentz tensor. This makes sense because the momentum operator, being a four-vector, has to transform according to a four-vector Lorentz transformation - and this holds off-forward.

A question was raised (BLT/Chen Ji) as to the correctness of the off-forward covariant form of the tensor decomposition. BLT in eqn (5.34) have the same "objectionable" form. That form is not covariant since the off-forward spinor product $\bar{u}\left(q^{\prime}\right) u(q)=\left(E_{q}^{\prime} E_{q}\right)^{\frac{1}{2}}$ is not covariant.So this form is not general. If one implements Lorentz transformations correctly on the spinors in going to a different frame one does not both change the momentum variable of the spinor and transform the spinor with $\Lambda_{\frac{1}{2}}(\eta)$ : one just does the latter (the Dirac wave-function transforms differently from its bare Fourier component, the Dirac eigenspinor). This is equivalent to transforming the Lorentz indices on the object/operator carrying Lorentz structure only in a covariant way.

However, this is fine since even though it is not covariant in the usual way, the form is correct in any given frame. And since it transforms covariantly with its tensor indices, the amplitude is frame-invariant when contracted with objects transforming contravariantly with their Lorentz indices. Incidentally, one would have the same "objection" on simple off-forward current form-factors as well, but it does not invalidate that decomposition. 


\section{Prelude comment on the meaning of Orbital Angular Momentum}

Alternatively, one can see this directly from the form of the operators that look like orbital AM for quarks (usual operator sandwiched between fermion fields) and gluons. These are interpreted, even relativistically, as operators OAM separately in any state. Harindranath and JM identify and separate these operators in an identical manner, whereas Ji differs in that he has a covariant derivative defining the OAM terms. Eventually, the separation of quark and gluon contributions, it is claimed, is a bit forced. Whether one uses the free-field operator or the one containing the covariant derivative, one is not isolating the quark contribution by itself. So, a case can be made for either decomposition in principle, and gauge-invariance needs to be considered. If the components are separately gauge-invariant that is good news, but we are thrown off the by fact that OAM and spin are not separately gauge-invariant in any of the above decompositions. Only in Ji's decomposition are quark OAM, quark spin, and total gluon AM separately gauge-invariant. We do not have any reason to believe that quark and gluon contributions are separately conserved, but gauge-invariance by no means implies separate conservation.

One has total angular momentum, which is sensibly defined in terms of an expectation value for all states. One can choose to understand OAM as the difference between AM and spin. For something at rest, it is clear that there is no OAM. A longitudinal boost should not change the OAM in that direction about any origin. If the particle were localized, then about the appropriate origin, it would not change at all in any direction. The original rest-frame state is a pure spin-state. What is the spin vector? The spin vector for any state is a vector of the 3 expectation values of the spin operator (Pauli matrices) in this state.(Transversely boosted canonical states are NOT eigenstates of helicity OR spin, but have expectation values of spin). So, OAM is what remains after one takes the difference of the total AM vector and this spin vector.

Further, BLT say that JM's derivation only yields their result in the longitudinal spin case, not in the case of transverse spin. They go on to show that there are further divergences if one uses the JM result for evaluating the matrix element in the case of transverse spin. The BLT result has the same compact form as above for whatever spin state is in question. BLT are supposedly puzzled by the existence of the delta term.

Peskin and Schroeder say $(\mathrm{Pg} 60)$ that the division of the J operator into spin and OAM is not straightforward relativistically, but BLT use it to mean that. They also go on to speak of 'spin in direction of OAM' etc in a state that is not longitudinal with 
spin .If one boosts from the rest frame in the direction of spin, the total AM cannot change since there can be no OAM along the boost direction as per this origin. And since the total is unchanged, all must be spin. This is analagous to a classical steady magnetic field remaining unchanged under longitudinal boosts. If the whole state is almost origin-independent where is this choice implicitly made? It is the origin choice contained implicitly in the phase from shifts/overlap etc., $e^{i p . x}$ and of course, whenever we write $x$ in the operators!

One would get a divergent result even in ordinary quantum mechanics if one tried to find the expectation value of angular momentum for an eigenstate of momentum (the state wouldn't be normalizable for a start). There were some concerns expressed over divergences of the expecation value of an observable following simple canonical rules of QFT. One uses Noether's theorem to obtain the AM operator in terms of the EM tensor and finds its expectation value. This result holds for any state in Hilbert space, and should not contain divergences when states are properly normalized. 
Appendix B

Derivation of the

Vorticity-Angular Momentum

Connection

Defining $T^{k}\left(p^{\prime}, p\right) \equiv\left\langle p^{\prime}\left|\Pi^{k}(0)\right| p\right\rangle, \Delta \equiv p-p^{\prime}$ and $A^{i j \alpha \beta} \equiv T^{k} \epsilon^{i j k} \delta^{\alpha} \delta^{\beta}$, 


$$
\begin{aligned}
& \left\langle\int d^{3} x \frac{x^{2}}{2} \nabla \times \vec{\Pi}(x)\right\rangle^{i}=\frac{1}{2} \lim _{k \rightarrow 0} \int d^{3} x d^{3} p d^{3} p^{\prime} \phi(p) \phi\left(p^{\prime}\right) e^{i k \cdot x}\left\langle p^{\prime}\left|x^{2} \partial^{j} \Pi^{k}(x)\right| p\right\rangle \epsilon^{i j k} \\
& =\frac{1}{2} \lim _{k \rightarrow 0} \int d^{3} x d^{3} p d^{3} p^{\prime} \phi(p) \phi\left(p^{\prime}\right) e^{i(k+\Delta) \cdot x} \Delta^{j} x^{2}\left\langle p^{\prime}\left|\Pi^{k}(0)\right| p\right\rangle \epsilon^{i j k} \\
& =\frac{1}{2} \lim _{k \rightarrow 0} \int d^{3} p d^{3} p^{\prime} \phi(p) \phi\left(p^{\prime}\right) e^{i(k+\Delta) \cdot x} d^{3} x \Delta^{j} x^{\alpha} x^{\beta} T^{k}\left(p^{\prime}, p\right) \epsilon^{i j k} \delta^{\alpha} \delta^{\beta} \\
& =\frac{1}{2} \lim _{k \rightarrow 0} \int d^{3} p d^{3} p^{\prime} \phi(p) \phi\left(p^{\prime}\right) \frac{\partial}{\partial k^{\alpha}} \frac{\partial}{\partial k^{\beta}} e^{i(k+\Delta) \cdot x} d^{3} x \Delta^{j} T^{k}\left(p^{\prime}, p\right) \epsilon^{i j k} \delta^{\alpha} \delta^{\beta} \\
& =\frac{1}{2} \lim _{k \rightarrow 0} \int d^{3} p d^{3} p^{\prime} \phi(p) \phi\left(p^{\prime}\right) \frac{\partial}{\partial k^{\alpha}} \frac{\partial}{\partial k^{\beta}} e^{i(k+\Delta) \cdot x} d^{3} x \Delta^{j} A^{i j \alpha \beta}\left(p^{\prime}, p\right) \\
& =\frac{1}{2} \lim _{k \rightarrow 0} \int d^{3} p d^{3} p^{\prime} \phi(p) \phi\left(p^{\prime}\right) \frac{\partial}{\partial k^{\alpha}} \frac{\partial}{\partial k^{\beta}} \delta^{(3)}(k+\Delta) \Delta^{j} A^{i j \alpha \beta}\left(p^{\prime}, p\right) \\
& =-\frac{1}{2} \lim _{k \rightarrow 0} \frac{\partial}{\partial k^{\alpha}} \frac{\partial}{\partial k^{\beta}} \int d^{3} p \phi(p) \phi^{*}(p+k) k^{j} A^{i j \alpha \beta}(p+k, p) \\
& =-\frac{1}{2} \lim _{k \rightarrow 0} \frac{\partial}{\partial k^{\alpha}} \frac{\partial}{\partial k^{\beta}} \int d^{3} p \phi(p) \phi^{*}(p+k) k^{j} A^{i j \alpha \beta}(p+k, p) \\
& =-\frac{1}{2} \lim _{k \rightarrow 0} \epsilon^{i j k} \delta_{\beta}^{\alpha}\left[\delta _ { j \alpha } \left(\int d^{3} p \phi(p)\left\langle p\left|\Pi^{k}(0)\right| p\right\rangle \frac{\partial}{\partial k_{\beta}} \phi^{*}(p+k)\right.\right. \\
& \left.+\int d^{3} p|\phi(p)|^{2} \frac{\partial}{\partial k_{\beta}}\left\langle p+k\left|\Pi^{k}(0)\right| p\right\rangle\right) \\
& +\delta_{j}^{\beta}\left(\int d^{3} p \phi(p)\left\langle p\left|\Pi^{k}(0)\right| p\right\rangle \frac{\partial}{\partial k_{\alpha}} \phi^{*}(p+k)\right. \\
& \left.\left.+\int d^{3} p|\phi(p)|^{2} \frac{\partial}{\partial k_{\alpha}}\left\langle p+k\left|\Pi^{k}(0)\right| p\right\rangle\right)\right] \\
& =-\lim _{k \rightarrow 0} \epsilon^{i j k}\left(\int d^{3} p \phi(p)\left\langle p\left|\Pi^{k}(0)\right| p\right\rangle \frac{\partial}{\partial k_{j}} \phi^{*}(p+k)\right. \\
& \left.+\int d^{3} p|\phi(p)|^{2} \frac{\partial}{\partial k_{j}}\left\langle p+k\left|\Pi^{k}(0)\right| p\right\rangle\right)
\end{aligned}
$$

This is to be exactly compared to (as in the wave packet relation of AM and the EMT),

$$
\begin{aligned}
<J^{\mu \lambda}> & =\lim _{\mathbf{k} \rightarrow \mathbf{0}}(-\mathbf{i}) \int \mathbf{d}^{3} \mathbf{p} \phi(\mathbf{p})\left\langle\mathbf{p}\left|\mathbf{T}_{\mathbf{B}}^{\mathbf{0 \lambda}}(\mathbf{0})\right| \mathbf{p}\right\rangle \frac{\partial}{\partial \mathbf{k}_{\nu}} \phi^{*}(\mathbf{p}+\mathbf{k})-(\lambda \leftrightarrow \nu) \\
& +(-\mathbf{i}) \int \mathbf{d}^{\mathbf{3}} \mathbf{p}|\phi(\mathbf{p})|^{2} \frac{\partial}{\partial \mathbf{k}_{\nu}}\left\langle\mathbf{p}+\mathbf{k}\left|\mathbf{T}_{\mathbf{B}}^{\mathbf{0}}(\mathbf{0})\right| \mathbf{p}\right\rangle-(\lambda \leftrightarrow \nu)
\end{aligned}
$$

It is understood above that $J^{12}$ means $J^{3}$ etc.

If, in particular, we replace $\overrightarrow{\Pi^{k}}$ by $-T_{B}^{0 k}$, we will get 


$$
\begin{aligned}
\left\langle\int d^{3} x \frac{-x^{2}}{2} \nabla \times T_{B e l}^{0 k}(x)\right\rangle^{i} & =\frac{1}{2}\left\langle J^{i}\right\rangle+\frac{1}{2}\left\langle J^{i}\right\rangle \\
& =\left\langle J^{i}\right\rangle
\end{aligned}
$$

Note that the derivative was taken outside the bra-ket in the first line. There are two reasons for this. In field theory, the coordinate space is in a sense disconnected from the Hilbert space (Fock space) and an operator which is a spatial derivative does not have much meaning inside the expectation value, just acting on the spatial coefficient of the ket. Thus, it applies to the expectation value of the operator, which is spatially dependent. Also, imagine translating a state along some axis, say, to the left. If this is done, both the bra and the ket are translated (or the operator is equivalently translated from both sides) to give physical meaning to the evaluation (which is a physical expectation value). The physically relevant quantity in Hilbert space is the expectation value and the spatial derivative cannot only apply to the ket in an isolated fashion. 


\section{Appendix C}

\section{Constructing the Transformation Matrices}

All the helicity amplitude dot products are of course Lorentz Invariant, and hence rotating to a frame where the helicity states point in an arbitrary direction will not change the amplitudes. However, it is instructive to start working in a generalized Breit Frame and then proceed to a frame with transverse momentum components and compare. The general Lorentz-transformation matrix, which transforms the polarization vectors, will act on the polarization vector that represents a state of definite spin along the $z$ axis $(+1,0$ or -1$)$ in the rest frame, and rotate it to polar angles $\phi$ and $\theta$. It will be a leftto-right product of the boost matrix taken along the $z$-axis, i.e. $\Lambda_{u}^{u^{\prime}}(-|p| \hat{z}), R_{y}(\theta)$ and $R_{z}(\phi)$, where $R_{i}$ denotes the 4-dimensional Lorentz matrix that rotates counterclockwise about axis $i$ by the specified angle.

The matrix is:

$$
T_{u}^{u^{\prime}}=R_{z}(\phi) R_{y}(\theta) \Lambda_{u}^{u^{\prime}}(-|p| \hat{z})
$$

where $|p|$ is the magnitude of the 3-momentum of the hadron and the indices on the right-hand-side have loosely been assigned to the boost matrix alone. 


$$
T_{u}^{u^{\prime}}=\left[\begin{array}{cccc}
1 & 0 & 0 & 0 \\
0 & \cos (t) \cos (\phi) & -\sin (\phi) & \cos (\phi) \sin (t) \\
0 & -\sin (\phi) \cos (t) & -\cos (\phi) & -\sin (\phi) \sin (t) \\
0 & -\sin (t) & 0 & -\cos (t)
\end{array}\right]\left[\begin{array}{cccc}
E_{1} / m & 0 & 0 & |p| / m \\
0 & 1 & 0 & 0 \\
0 & 1 & 0 & 0 \\
|p| / m & 0 & 0 & E_{1} / m
\end{array}\right]
$$

where $\cos (t)=\frac{p_{3}}{|p|}, \tan (\phi)=\frac{p_{2}}{p_{1}}, m$ is the mass of the deuteron and $E_{1}=\sqrt{m^{2}+|p|^{2}}$ its energy. This gives

$$
T_{u}^{u^{\prime}}=\left[\begin{array}{cccc}
\frac{E_{1}}{m} & 0 & 0 & \frac{|p|}{m} \\
\frac{b|p|}{a} & \frac{p_{3}}{a|p|} & -\frac{p_{2}}{p_{1} a} & \frac{E_{1} b}{m a} \\
\frac{p_{2}|p| b}{m p_{1} a} & \frac{p_{2} p_{3}}{p_{1} a|p|} & 1 / a & \frac{p_{2} E_{1} b}{m p_{1} a} \\
\frac{p_{3}}{m} & -b & 0 & \frac{p_{3} E_{1}}{m|p|}
\end{array}\right]
$$

where $a=\sqrt{1+\left(\frac{p_{2}}{p_{1}}\right)^{2}}$ and $b=\sqrt{1-\left(\frac{p_{3}}{|p|}\right)^{2}}$. Note that to obtain a polarization vector with helicity in exactly the opposite direction, one does not simply multiply by a negative $3 \times 3$ identity matrix as for ordinary 4-vectors like momentum, which has spatial components only in the simplest plane of rotation that one would use to obtain its parity transform. One formally rotates the rest frame, spin-up-along- $z$ polarization vector by $\pi$ about the $x$-axis, then by $\theta$ about $y$ and last by $\phi$ about $z$, the last two counterclockwise again. Thus one obtains a state that is exactly opposite to the above in our frame.

$$
\begin{gathered}
T_{v}^{v^{\prime}}=R_{z}(\phi) R_{y}(\theta) R_{x}(\pi) \Lambda_{v}^{v^{\prime}}(-|p| \hat{z}) \\
T_{v}^{v^{\prime}}=\left[\begin{array}{cccc}
\frac{E_{1}}{m} & 0 & 0 & \frac{|p|}{m} \\
\frac{b|p|}{a} & \frac{p_{3}}{a|p|} & -\frac{p_{2}}{p_{1} a} & \frac{E_{1} b}{m a} \\
-\frac{p_{2}|p| b}{m p_{1} a} & -\frac{p_{2} p_{3}}{p_{1} a|p|} & -1 / a & -\frac{p_{2} E_{1} b}{m p_{1} a} \\
-\frac{p_{3}}{m} & b & 0 & -\frac{p_{3} E_{1}}{m|p|}
\end{array}\right],
\end{gathered}
$$

Then we have $S_{u v}^{u^{\prime} v^{\prime}}=T_{u}^{u^{\prime}} T_{v}^{v^{\prime}}$ for this frame.

It is clear that $p_{z}$ appears differently in the transformation because of the choice of initial polarization vector. By the way, if there is a phase from a rotation that we have not accounted for it will only be an overall phase due to the decomposition having the 
same objects to be transformed identically. 


\section{Bibliography}

[1] Xiang-Dong Ji. Off forward parton distributions. J.Phys., G24:1181-1205, 1998. doi: 10.1088/0954-3899/24/7/002.

[2] Xiang-Dong Ji. Gauge invariant decomposition of nucleon spin and its spin - off. Phys.Rev.Lett., 78:610-613, 1997. doi: 10.1103/PhysRevLett.78.610.

[3] Hikmat BC and Matthias Burkardt. Orbital Angular Momentum in Scalar Diquark Model and QED. Few Body Syst., 52:389-395, 2012. doi: 10.1007/ s00601-011-0259-0.

[4] M. Diehl. Generalized parton distributions. Phys.Rept., 388:41-277, 2003. doi: 10.1016/j.physrep.2003.08.002.

[5] R.L. Jaffe and Aneesh Manohar. The G(1) Problem: Fact and Fantasy on the Spin of the Proton. Nucl.Phys., B337:509-546, 1990. doi: 10.1016/0550-3213(90)90506-9.

[6] Gary R. Goldstein, J. OsvaldoGonzalez Hernandez, and Simonetta Liuti. Flexible Parametrization of Generalized Parton Distributions from Deeply Virtual Compton Scattering Observables. Phys.Rev., D84:034007, 2011. doi: 10.1103/PhysRevD.84. 034007.

[7] Elliot Leader. On the controversy concerning the definition of quark and gluon angular momentum. Phys.Rev., D83:096012, 2011. doi: 10.1103/PhysRevD.85. 039905,10.1103/PhysRevD.83.096012.

[8] Matthias Burkardt. Transverse Force on Quarks in DIS. 2008.

[9] A. Harindranath and Rajen Kundu. On Orbital angular momentum in deep inelastic scattering. Phys.Rev., D59:116013, 1999. doi: 10.1103/PhysRevD.59.116013.

[10] Swadhin K. Taneja, Kunal Kathuria, Simonetta Liuti, and Gary R. Goldstein. Angular momentum sum rule for spin one hadronic systems. Phys.Rev., D86:036008, 2012. doi: 10.1103/PhysRevD.86.036008.

[11] G.M. Shore and B.E. White. The Gauge invariant angular momentum sum rule for the proton. Nucl.Phys., B581:409-431, 2000. doi: 10.1016/S0550-3213(00)00288-1. 
[12] Franz Gross and Ronald A. Gilman. The Deuteron: A Mini review. AIP Conf.Proc., 603:55-68, 2001. doi: 10.1063/1.1436589.

[13] F. Cano and B. Pire. Deep electroproduction of photons and mesons on the deuteron. Eur.Phys.J., A19:423-438, 2004. doi: 10.1140/epja/i2003-10127-x.

[14] Edgar R. Berger, F. Cano, M. Diehl, and B. Pire. Generalized parton distributions in the deuteron. Phys.Rev.Lett., 87:142302, 2001. doi: 10.1103/PhysRevLett.87. 142302 .

[15] Xiangdong Ji, Xiaonu Xiong, and Feng Yuan. Transverse Polarization of the Nucleon in Parton Picture. Phys.Lett., B717:214-218, 2012. doi: 10.1016/j.physletb. 2012.09.027.

[16] Elliot Leader and Cedric Lorce. Comment on 'Proton Spin Structure from Measurable Parton Distributions' by Ji, Xiong and Yuan (PRL109, 152005 (2012)). 2012.

[17] D.V. Kiptily and M.V. Polyakov. Genuine twist three contributions to the generalized parton distributions from instantons. Eur.Phys.J., C37:105-114, 2004. doi: 10.1140/epjc/s2004-01957-3.

[18] Xiangdong Ji, Jian-Hui Zhang, and Yong Zhao. Physics of Gluon Helicity Contribution to Proton Spin. 2013.

[19] B.L.G. Bakker, E. Leader, and T.L. Trueman. A Critique of the angular momentum sum rules and a new angular momentum sum rule. Phys.Rev., D70:114001, 2004. doi: 10.1103/PhysRevD.70.114001.

[20] Yoshitaka Hatta, Kazuhiro Tanaka, and Shinsuke Yoshida. Twist-three relations of gluonic correlators for the transversely polarized nucleon. JHEP, 1302:003, 2013. doi: 10.1007/JHEP02(2013)003.

[21] M. Burkardt, A. Miller, W. -D. Nowak, Rept. Prog. Phys. 73, 016201 (2010).

[22] R. L. Jaffe and A. Manohar, Nucl. Phys. B 337, 509 (1990).

[23] X. D. Ji, Phys. Rev. Lett. 78, 610 (1997)

[24] X. S. Chen, X. F. Lu, W. M. Sun, F. Wang and T. Goldman, Phys. Rev. Lett. 100, 232002 (2008); ibid Phys. Rev. D 83, 071901 (2011).

[25] M. Wakamatsu, Eur. Phys. J. A 44 (2010) 297; ibid Phys. Rev. D 84, 037501 (2011).

[26] E. Leader, Phys. Rev. D 83, 096012 (2011) [Erratum-ibid. D 85, 039905 (2012)] 
[27] B. L. G. Bakker, E. Leader and T. L. Trueman, Phys. Rev. D 70, 114001 (2004)

[28] M. Burkardt and H. BC, Phys. Rev. D 79, 071501 (2009)

[29] E. R. Berger, F. Cano, M. Diehl and B. Pire, Phys. Rev. Lett. 87, 142302 (2001)

[30] Z. Chen and X. d. Ji, Phys. Rev. D 71, 016003 (2005)

[31] Ph. Hagler, Phys. Lett. B 594, 164 (2004)

[32] G. R. Goldstein, J. O. G. Hernandez and S. Liuti, arXiv:1201.6088 [hep-ph].

[33] G.R. Goldstein, K. Kathuria, S. Liuti, and S.K. Taneja in preparation.

[34] G. R. Goldstein, J. O. G. Hernandez and S. Liuti, Phys. Rev. D 84, 034007 (2011).

[35] S. Liuti, S. K. Taneja, Phys. Rev. C72, 034902 (2005).

[36] R. A. Gilman, F. Gross, J. Phys. G28, R37 (2002).

[37] M. Garcon and J. W. Van Orden, Adv. Nucl. Phys. 26, 293 (2001)

[38] C. Ciofi degli Atti and S. Liuti, Phys. Rev. C 41, 1100 (1990).

[39] E. Leader, A. V. Sidorov, D. B. Stamenov, Phys. Rev. D73, 034023 (2006).

[40] O. Hen, E. Piasetzky, R. Shneor, L. B. Weinstein and D. W. Higinbotham, arXiv:1109.6197 [hep-ph].

[41] A. W. Thomas, Int. J. Mod. Phys. E 18, 1116 (2009)

[42] P. Hoodbhoy, R. L. Jaffe, A. Manohar, Nucl. Phys. B312, 571 (1989).

[43] A. Kirchner, D. Mueller, Eur. Phys. J. C32, 347 (2003). 